\title{
AUTOMATED SG-DBR TUNABLE LASER CALIBRATION OPTIMIZED FOR OPTICAL COHERENCE TOMOGRAPHY APPLICATIONS
}

\author{
A Thesis Presented to the \\ Electrical Engineering Department Faculty of \\ California Polytechnic State University, San Luis Obispo \\ In Partial Fulfillment \\ of the Requirements for the Degree \\ Master of Science in Electrical Engineering
}

By

Christopher Louis Chiccone

June 2011 
(C) 2011

Christopher Louis Chiccone

ALL RIGHTS RESERVED 


\section{Committee Membership}

Title:

Author:

Date Submitted:
Automated SG-DBR Tunable Laser Calibration Optimized for Optical Coherence Tomography Applications

Christopher Louis Chiccone

June 2011

Committee Chair: $\quad$ Dr. Dennis Derickson

Committee Member: Dr. Dale Dolan

Committee Member: Dr. Wayne Pilkington 


\begin{abstract}
Title: $\quad$ Automated SG-DBR Tunable Laser Calibration Optimized for Optical Coherence Tomography Applications
\end{abstract}

Author: Christopher Louis Chiccone

SG-DBR lasers look to solve many problems associated with present OCT sources by being cost effective, smaller in size, more robust, and by operating at faster repetition rates. Swept Source Optical Coherence Tomography (SS-OCT) requires a tunable laser source that exhibits linear frequency sweeps, large frequency spans, and high repetition rates. This work accomplishes this by using four synchronized waveforms sent to the input of a Sampled Grating-Distributed Bragg Reflector (SGDBR) laser. Three mirrors control the wavelength, while an internal semiconductor optical amplifier controls the laser output power. In dealing with this complicated tuning mechanism, a manual sweep calibration is too time-consuming. This thesis demonstrates an efficient method for automating the calibration of tunable SG-DBR lasers by implementing a gain medium voltage sensing algorithm, as opposed to the previous inefficient manual efforts.

Experimental OCT tests are also performed by utilizing a Mach-Zehnder interferometer as a device under test to verify the accuracy of the laser calibration methodology. The OCT response to a single reflection event is measured over a range of repetition rates. A method to reduce these spurious display responses caused by wavelength stitching imperfections is implemented through a self-generating optical clock. 


\section{Acknowledgements}

I would like to express my deepest gratitude to Dr. Dennis Derickson. Without his guidance and contributions to the project, this thesis would not be possible. In addition to the research, he has furthered my knowledge through countless lectures, which has provided a great foundation to start my career.

Great appreciation goes to Brandon George and Shane O'Connor for their previous work on the SG-DBR project. Their contributions provided the much needed groundwork for my progression throughout this experience. Furthermore, I would like to thank Dr. Dale Dolan and Dr. Wayne Pilkington for participating in my thesis committee and especially Scott Marshall for providing software assistance. Also, thank you to Jason Ensher and Jonathan Huber from Global Test who also contributed greatly to this project.

I would like to quickly thank my friends and family, particularly Katie and Lindsay, who gave me the much needed escape from the endless hours in lab by giving me an outlet to express myself outside of school.

Last, but certainly not least, I would like to thank my parents for their unbelievable amount of support throughout my entire life. By their unwavering belief in my talents, they've allowed me to grow and become the person that I've always aspired to be. 


\section{Table of Contents}

LIST OF TABLES ............................................................................................................................................ VIII

LIST OF FIGURES ………….................................................................................................................. IX

CHAPTER 1 - INTRODUCTION................................................................................................................1

CHAPTER 2 - SWEPT SOURCE OPTICAL COHERENCE TOMOGRAPHY ..................................3

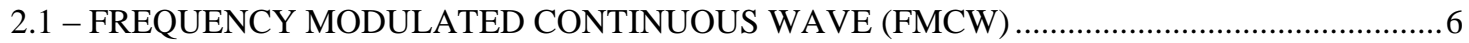

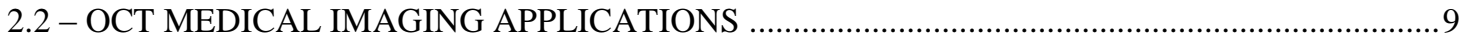

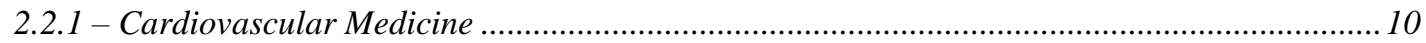

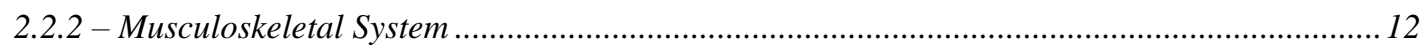

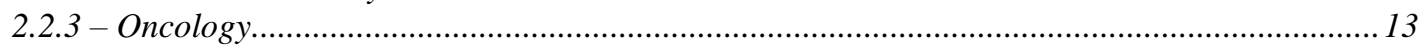

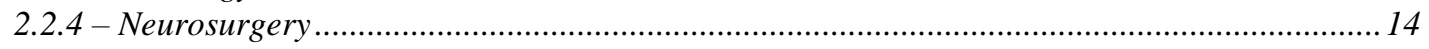

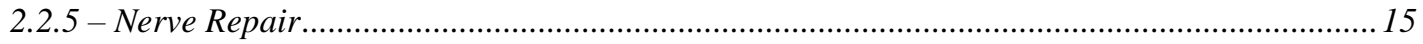

CHAPTER 3 - SG-DBR TUNABLE LASER ..........................................................................................16

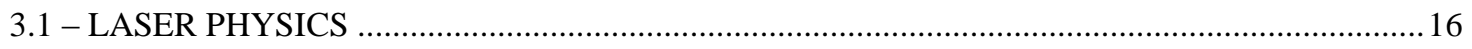

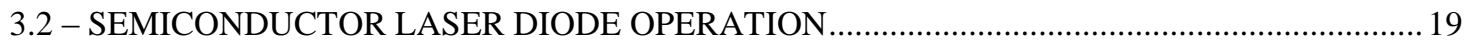

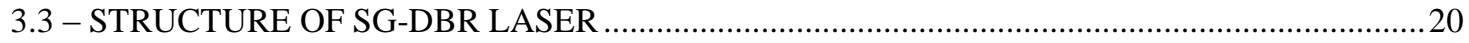

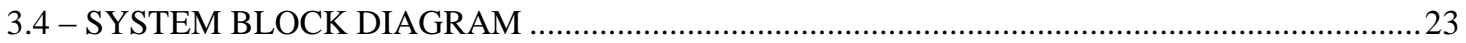

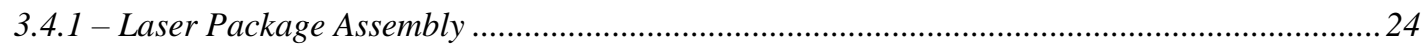

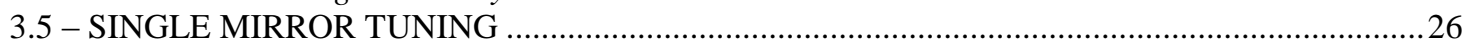

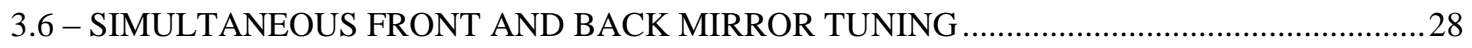

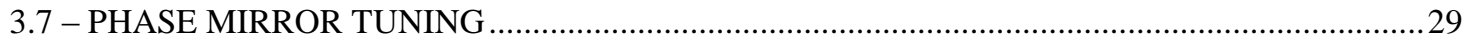

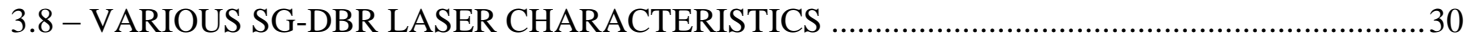

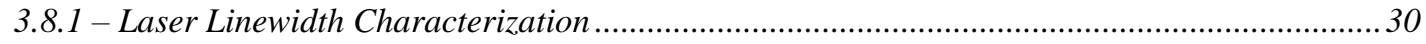

3.8.2 - Tuning Pad Side Mode Suppression Ratio (SMSR) .............................................................. 37

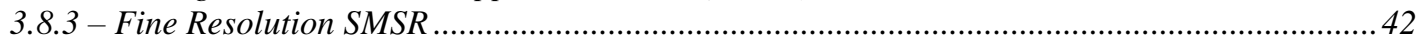

CHAPTER 4 - GAIN MEDIUM VOLTAGE IMPLICATIONS.........................................................43

4.1 - INSTANTANEOUS VOLTAGE CHANGE AT WAVELENGTH MODE TRANSITIONS .......43

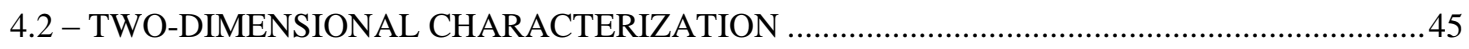

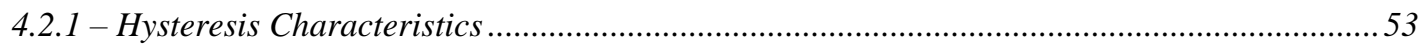

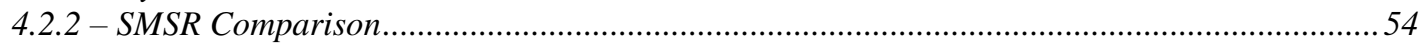

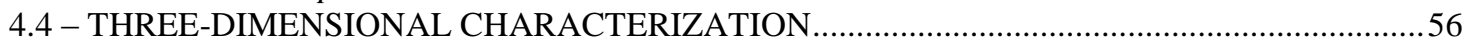

CHAPTER 5 -AUTOMATED LASER CALIBRATION ......................................................................60

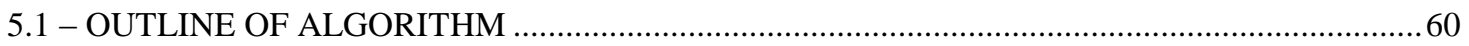

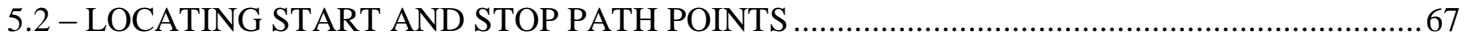

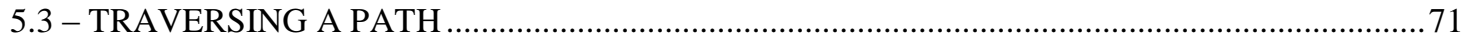

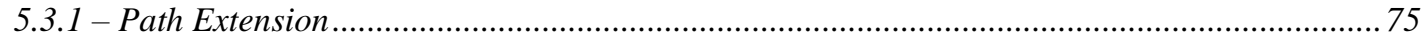

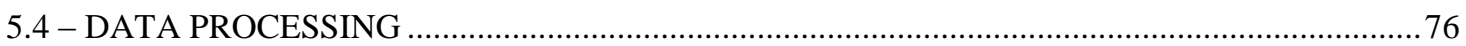

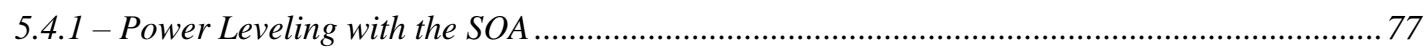

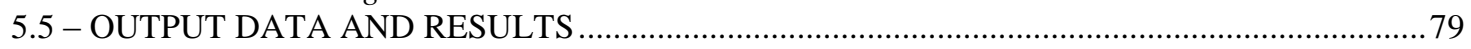

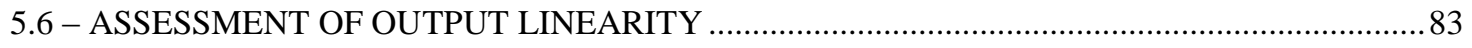

5.6.1 - Statistical Analysis of Zero-Crossings of Interference Pattern................................................ 83

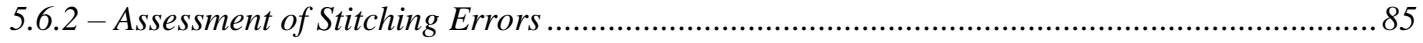


CHAPTER 6 - SWEPT SOURCE OCT EXPERIMENTAL RESULTS ........................................86

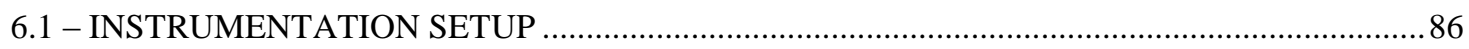

6.2 - IMPROVED SPECTRAL RESPONSE WITH REMOVAL OF STITCHING ERRORS.............88

6.3 - SPECTRAL BROADENING AS A FUNCTION OF SWEEP RATE ...................................90

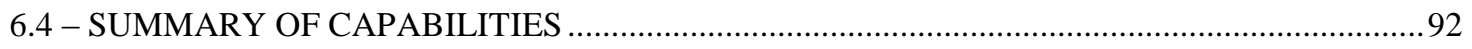

CHAPTER 7 - CONCLUSION AND FUTURE WORK.................................................................................94

BIBLIOGRAPHY

APPENDIX A - JDSU SG-DBR PIN-OUT AND OPERATING CURRENT LEVELS......................98 


\section{List of Tables}

TABLE 3.1: DIFFERENCE WAVELENGTH AND FREQUENCY DATA FOR TWO INTERFEROMETERS .............................. 34

TABLE 3.2: DATA REPRESENTING THE SIDEBAND DEVIATION AS A FUNCTION OF CURRENT …….......................... 40

TABLE 4.1: SUMMARY OF CALCULATED THRESHOLD FOR L-BAND WITH CONSTANT INCREMENT SIZE ..................55

TABLE 4.2: SUMMARY OF CALCULATED THRESHOLD FOR C-BAND WITH VARIABLE INCREMENT SIZE ...................52

TABLE 5.1: EXAMPLE DATA STRUCTURE USED TO PROCESS AND SAVE DATA ………............................................. 76

TABLE 5.2: SUMMARY OF C-BAND PERFORMANCE COMPARED TO THE MANUAL CALIBRATION …………………......8 82

TABLE 5.3: QUALITATIVE DETERMINATION OF STITCHING IMPERFECTIONS ……………...................................... 85

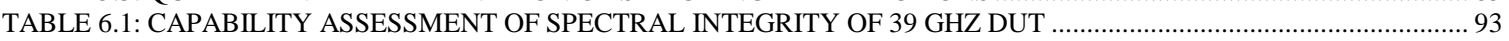




\section{List of Figures}

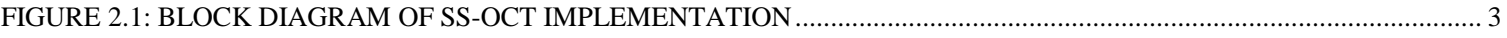
FIGURE 2.2: CORRESPONDING WAVEFORMS WITH LOCATIONS DEPICTED IN FIGURE (2.1) ........................................ 4 FIGURE 2.3: FMCW GRAPH OF TRANSMITTED AND RECEIVED SIGNALS FROM STATIONARY TARGET ...................... 6 FIGURE 2.4: OCT IMAGE OF HEAVILY CALCIFIED PLAQUE WITH CORRESPONDING HISTOPATHOLOGY ................... 10 FIGURE 2.5: STENT USED IN INTERVENTIONAL OCT CARDIOLOGY ................................................................... 11

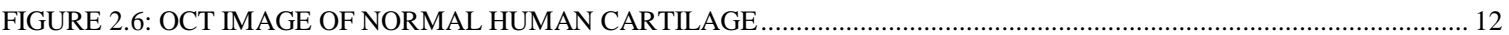

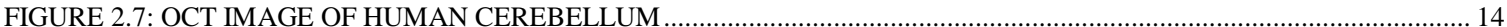

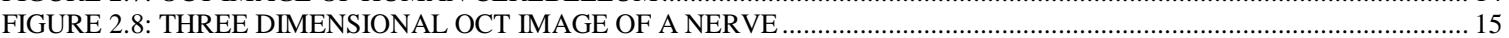

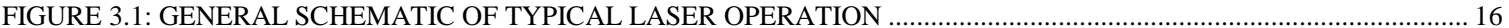
FIGURE 3.2: DISPLAYS STIMULATED EMISSION CAUSED BY ELECTRON MOVEMENT ............................................ 18 FIGURE 3.3: ENERGY BAND DIAGRAM OF RECOMBINATION TRANSITION ACROSS FERMI LEVEL .......................... 19 FIGURE 3.4: TOP VIEW OF THE LASER SHOWING THE FIVE PRIMARY SECTIONS ....................................................... 20 FIGURE 3.5: CROSS SECTION OF THE LASER SHOWING THE VARIOUS BANDGAP AREAS ......................................... 21 FIGURE 3.6: BRAGG REFLECTORS AND THEIR REFLECTIVITY FOR THE FRONT MIRROR AND BACK MIRROR ......... 22 FIGURE 3.7: BLOCK DIAGRAM OF SG-DBR LASER WITH CORRESPONDING INPUTS AND OUTPUTS .......................... 23

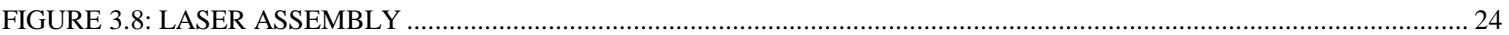
FIGURE 3.9: OPTICAL ISOLATOR.

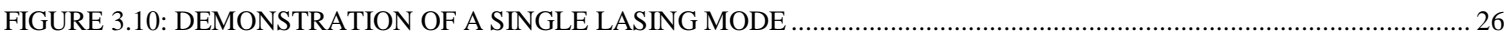
FIGURE 3.11: QUALITATIVE DEMONSTRATION OF CHANGING THE BIAS CURRENT TO THE FRONT MIRROR........... 27 FIGURE 3.12: QUALITATIVE DEMONSTRATION OF CHANGING THE BIAS CURRENT TO THE FRONT AND BACK...... 28 FIGURE 3.13: ADJUSTING THE CURRENT TO THE PHASE SECTION OF THE LASER …................................................. 29

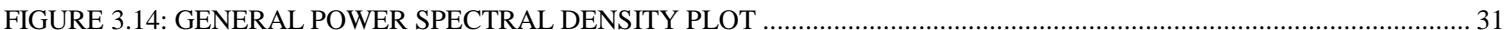

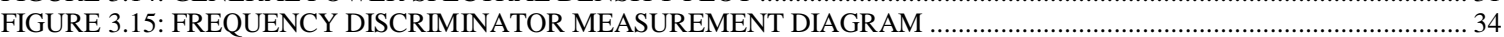
FIGURE 3.16: 2.5 PM MACH-ZEHNDER INTERFEROMETER UPPER RANGE AMPLITUDE VALUES ............................... 34 FIGURE 3.17: 2.5 PM MACH-ZEHNDER INTERFEROMETER MIDRANGE AMPLITUDE VALUES ......................................... 35 FIGURE 3.18: 1.0 PM MACH-ZEHNDER INTERFEROMETER UPPER RANGE AMPLITUDE VALUES .............................. 35 FIGURE 3.19: 1.0 PM MACH-ZEHNDER INTERFEROMETER MIDRANGE AMPLITUDE VALUES .................................... 36 FIGURE 3.20: EXAMPLE OF A LOWER SIDEBAND WITH SMALL SIDE MODE SUPPRESSION RATIO .......................... 38 FIGURE 3.21: CONTOUR PLOT FOR L-BAND LASER SHOWING NORMAL RESOLUTION CHARACTERIZATION ........... 39 FIGURE 3.22: GRAPH OF SIDEBAND MAGNITUDE AS A FUNCTION OF FRONT AND BACK MIRROR CURRENT .......... 39 FIGURE 3.23: GRAPH OF FINE RESOLUTION SIDEBAND MAGNITUDE AS A FUNCTION OF FM AND BM CURRENT ... 42 FIGURE 4.1: CONTOUR PLOT FOR L-BAND LASER SHOWING NORMAL RESOLUTION WITH MODE HOPS .................. 44 FIGURE 4.2: VOLTAGE RESPONSE AND WAVELENGTH WITH A LINEAR INCREASE OF FM AND BM CURRENT ........ 45

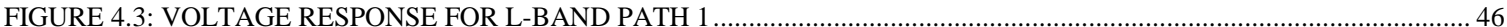

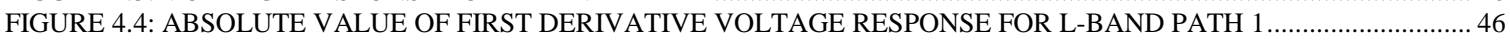
FIGURE 4.5: ABSOLUTE VALUE OF SECOND DERIVATIVE VOLTAGE RESPONSE FOR L-BAND PATH 1...................... 47

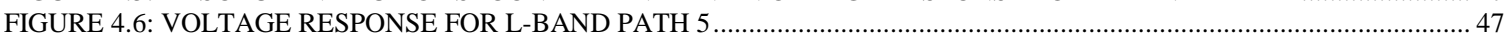
FIGURE 4.7: ABSOLUTE VALUE OF FIRST DERIVATIVE VOLTAGE RESPONSE FOR L-BAND PATH 5.............................. 48 FIGURE 4.8: ABSOLUTE VALUE OF SECOND DERIVATIVE VOLTAGE RESPONSE FOR L-BAND PATH 5....................... 48 FIGURE 4.9: ABSOLUTE VALUE OF FIRST DERIVATIVE VOLTAGE RESPONSE FOR C-BAND PATH $1 \ldots . . . . . . . . . . . . . . . . . . . . . . . . .49$ FIGURE 4.10: ABSOLUTE VALUE OF SECOND DERIVATIVE VOLTAGE RESPONSE FOR C-BAND PATH 1 ........................ 49 FIGURE 4.11: ABSOLUTE VALUE OF FIRST DERIVATIVE VOLTAGE RESPONSE FOR C-BAND PATH 5............................ 50 FIGURE 4.12: ABSOLUTE VALUE OF SECOND DERIVATIVE VOLTAGE RESPONSE FOR C-BAND PATH 5 .................... 50 FIGURE 4.13: ABSOLUTE VALUE OF SECOND DERIVATIVE VOLTAGE RESPONSE FOR L-BAND PATH $8 . . . . . . . . . . . . . . . . . . . . . .52$ FIGURE 4.14: HYSTERESIS CHARACTERISTICS FOR TWO-DIMENSIONAL MIRROR TUNING ................................... 53 FIGURE 4.15: SMSR RELATED TO THE VOLTAGE RESPONSE ACROSS A SINGLE TUNING PAD (NORMAL RES.)......... 54 FIGURE 4.16: SMSR RELATED TO THE VOLTAGE RESPONSE ACROSS A SINGLE TUNING PAD (FINE RES.) ................ 55 FIGURE 4.17: THREE-DIMENSIONAL (MODE MAP) PLOT OF WAVELENGTH VS CURRENTS (PM CURRENT = 0 mA) ... 57 FIGURE 4.18: THREE-DIMENSIONAL (MODE MAP) PLOT OF VOLTAGE VS CURRENTS $(\mathrm{PM} \mathrm{CURRENT} \mathrm{=} 0 \mathrm{~mA}) . . . . . . . . . .57$ FIGURE 4.19: THREE-DIMENSIONAL (MODE MAP) PLOT OF VOLTAGE VS CURRENTS (PM CURRENT = 5 mA) ........... 58 FIGURE 4.20: THREE-DIMENSIONAL (MODE MAP) PLOT OF VOLTAGE VS CURRENTS $(\mathrm{PM} \mathrm{CURRENT} \mathrm{=} 10 \mathrm{~mA}) \ldots \ldots . . . . .58$ FIGURE 4.21: THREE-DIMENSIONAL (MODE MAP) PLOT OF VOLTAGE VS CURRENTS (PM CURRENT = 15 mA)......... 59 FIGURE 4.22: THREE-DIMENSIONAL (MODE MAP) PLOT OF VOLTAGE VS CURRENTS $(\mathrm{PM} \mathrm{CURRENT} \mathrm{=} 20 \mathrm{~mA}) \ldots \ldots \ldots . . . .59$ FIGURE 5.1: BLOCK DIAGRAM OF AUTOMATED CALIBRATION INSTRUMENTATION SETUP ..................................... 61

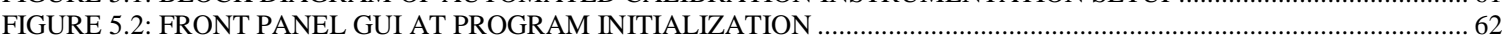

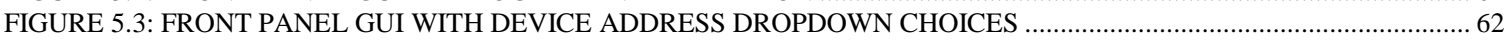

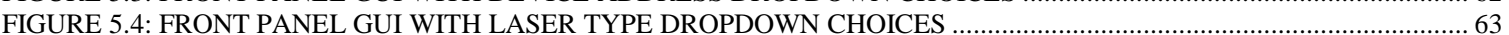
FIGURE 5.5: LASER TYPE SETTINGS FILE CORRESPONDING TO THE CHOICES IN FIGURE (5.4) ................................ 63 FIGURE 5.6: FRONT PANEL GUI WITH ADDITIONAL LASER TYPE DROPDOWN CHOICE .............................................64 FIGURE 5.7: LASER TYPE SETTINGS FILE CORRESPONDING TO THE NEW CHOICE IN FIGURE (5.6).......................... 64 FIGURE 5.8: HIGH LEVEL SOFTWARE FLOWCHART FOR THE AUTOMATED CALIBRATION PROCESS ........................... 66 FIGURE 5.9: VISUAL REPRESENTATION OF LOCATING THE START AND STOP POINTS OF PATHS ............................ 67

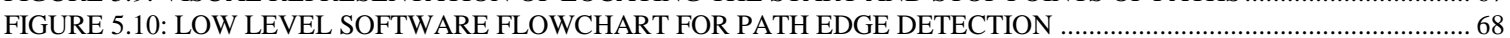
FIGURE 5.11: SOFTWARE FLOWCHART FOR LOCATING THE START POINTS OF A PATH....................................... 69

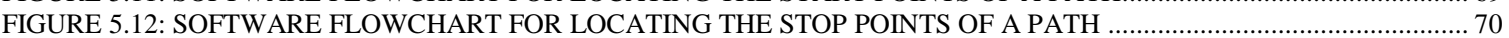
FIGURE 5.13: SOFTWARE FLOWCHART FOR THRESHOLD CALCULATION ….......................................................... 71

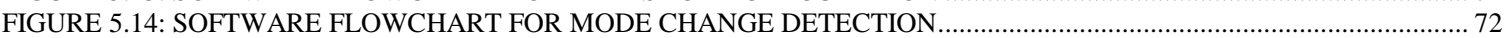


FIGURE 5.15: VISUAL REPRESENTATION OF LOCATING THE CENTER OF A PAD 73 FIGURE 5.16: SOFTWARE FLOWCHART FOR TRAVERSING A PATH........................................................................ FIGURE 5.17: VISUAL REPRESENTATION OF VARIOUS FUNCTIONS WHILE TRAVERSING A PATH ................................ 74 FIGURE 5.18: VISUAL REPRESENTATION OF PATH EXTENSION. FIGURE 5.19: GRAPH DEPICTING THE RELATIONSHIP BETWEEN GAIN AND CORRESPONDING SOA VALUES.... FIGURE 5.20: INPUT NORMALIZED BM ARBITRARY WAVEFORM DATA

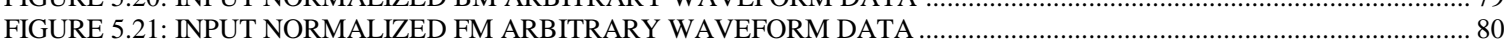
FIGURE 5.22: INPUT NORMALIZED PM ARBITRARY WAVEFORM DATA

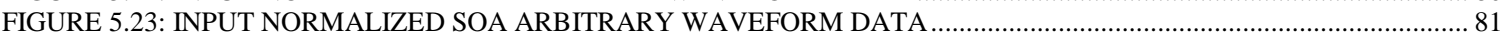

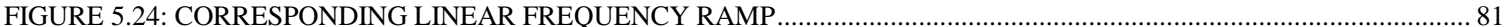
FIGURE 5.25: OUTPUT FREQUENCY RAMP FROM THE OSA WITH A 1KHZ REPETITION RATE ......................................8 82 FIGURE 5.26: INTERFERENCE PATTERN PRODUCED BY A 7.1 GHZ FSR INTERFEROMETER ……...............................83 FIGURE 5.27: HISTOGRAM OF TIME BETWEEN ZERO-CROSSINGS ………........................................................ 84 FIGURE 5.28: UNDERLAP STITCHING IMPERFECTION CAUSED BY FAST-SWITCHING WAVEFORM...........................85 FIGURE 6.1: BLOCK DIAGRAM OF INSTRUMENTATION SETUP FOR SELF-GENERATION OF CLOCK .......................... 86 FIGURE 6.2: BLOCK DIAGRAM OF INSTRUMENTATION SETUP FOR DUT SAMPLING ….............................................. 87 FIGURE 6.3: GRAPH OF INTERFERENCE PATTERNS FOR DUT AND OPTICAL SAMPLING CLOCK …............................87 FIGURE 6.4: TWO FALSE SQUARE WAVE PULSES GENERATED BY STITCHING ERRORS ……....................................8 88 FIGURE 6.5: FFT RESPONSE OF 39 GHZ DUT WITH NO STITCHING ERRORS REMOVED IN CLOCK..............................89 FIGURE 6.6: FFT RESPONSE OF 39 GHZ DUT WITH ALL STITCHING ERRORS REMOVED IN CLOCK .............................89 FIGURE 6.7: FFT RESPONSE OF 39 GHZ DUT WITH A 200 HZ REPETITION RATE ...................................................90 FIGURE 6.8: FFT RESPONSE OF 39 GHZ DUT WITH A 200 HZ REPETITION RATE IN DB................................................... 91

FIGURE 6.9: FFT RESPONSE OF 39 GHZ DUT WITH A 3 KHZ REPETITION RATE .....................................................91 


\section{CHAPTER 1 - Introduction}

The Sampled Grating-Distributed Bragg Reflector (SG-DBR) laser provides a compelling solution suitable for Swept Source Optical Coherence Tomography (SSOCT). SG-DBR lasers look to solve many problems associated with present OCT sources by being cost effective, smaller in size, more robust, and by operating at faster repetition rates.

Chapter 2 establishes a basis for SS-OCT beginning with the fundamental principles of frequency modulated continuous wave reflectometry. Example OCT medical applications, particularly cardiovascular medicine, are provided to demonstrate its competitive advantage against existing imaging systems.

The SG-DBR laser functionality and control process are described in chapter 3 which begins with an explanation of laser physics. Theoretical semiconductor laser operation, ranging from the Pauli Exclusion Principle to recombination transitions across Fermi levels, is included. The laser linewidth and side mode suppression ratios (SMSR) are also experimentally tested.

Chapter 4 delves into the gain medium voltage characterization as a function of two and three dimensional tuning. Instantaneous voltage spikes occur at wavelength mode intersections which provide sufficient information to sense constant wavelength pads automatically. The SMSR is also tested as a function of gain medium voltage to allow for optimal tuning paths.

The automated calibration which is developed in Python is extensively described in chapter 5. The general algorithm is outlined and specific functions within the program are explained. The success of implementing the algorithm is proven by calibrating a 
C-Band laser. The automated process dramatically reduces calibration time, increases bandwidth, and improves power leveling when compared to previous manual efforts, which are extremely time inefficient.

Finally in chapter 6 , experimental OCT tests are performed by utilizing a MachZehnder interferometer as a device under test. Repetition rates of a single reflection event are analyzed in comparison to the spectral broadening in the FFT response. A method to reduce these spurious display responses caused by various wavelength stitching imperfections is implemented through a self-generating optical clock and a LabVIEW deglitching program. A $7.1 \mathrm{GHz}$ free spectral range interferometer is used to generate this optical sampling clock which feedbacks into an A/D converter. Overall system performance is assessed based on OCT measurement capabilities. 


\section{CHAPTER 2 - Swept Source Optical Coherence Tomography}

SS-OCT is an imaging technique that uses optical coherent light to measure reflectivity versus position. The near infrared region of the electromagnetic spectrum is used in SS-OCT. Like many sensing procedures, SS-OCT utilizes the reflected beams of electromagnetic waves to provide a tomography of the region or device under test (DUT). For medical imaging, SS-OCT uses interferometry to extract distance information of the structures being imaged [1]. In general, reflected waves create a time domain interference pattern with the transmitted waves. An FFT can then be applied to infer distance data. Typically, maximum depth profiles extend to a few millimeters beneath biological samples. Figure (2.1) shows a block diagram of a typical SS-OCT implementation with a Mach-Zehnder interferometer used to generate an optical clock.

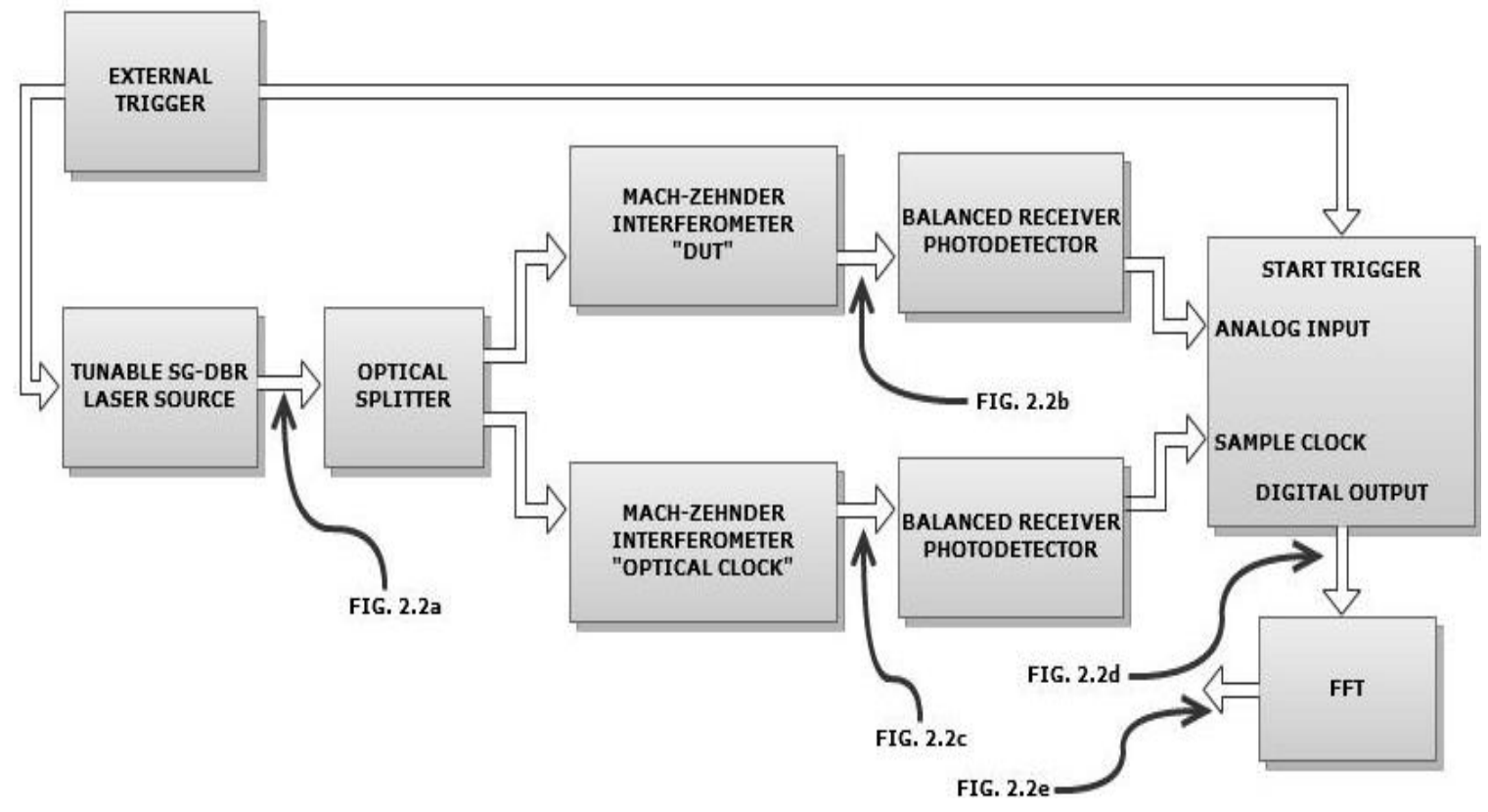

Figure 2.1: Block diagram of SS-OCT implementation 

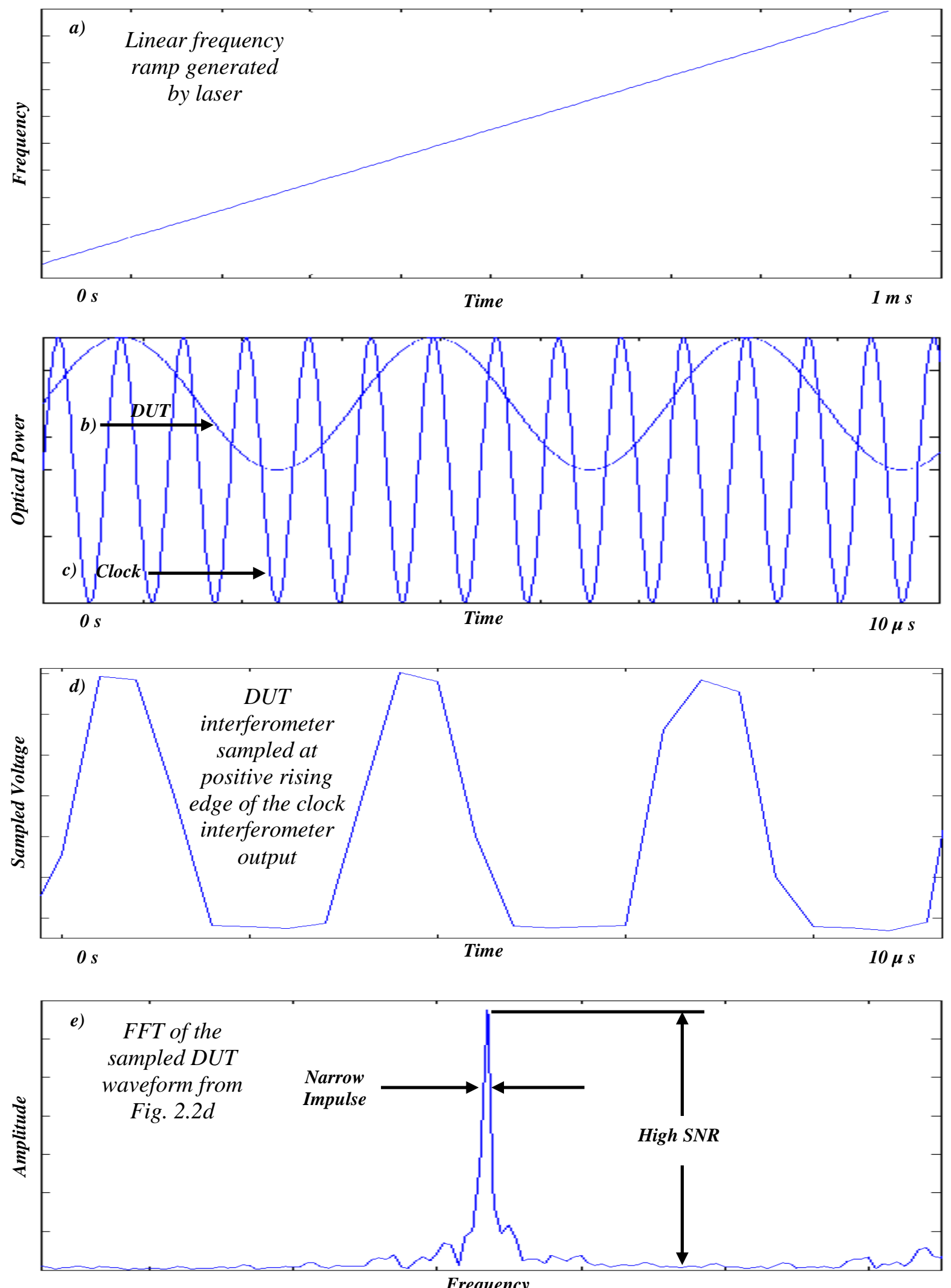

Figure 2.2: Corresponding waveforms with locations depicted in figure (2.1) 
The waveforms depicted in figure (2.2) correspond to the locations referenced in figure (2.1). SS-OCT is implemented with a tunable laser source that linearly increases in optical frequency over time as seen in figure (2.2a). This source, a SG-DBR laser, is thoroughly explained in chapter 3. This optical source is emitted into two Mach-Zehnder interferometers through an optical splitter. Both interferometers produce sinusoidal interference patterns, one at a significantly higher frequency as seen in figure (2.2c), which is used as an optical sampling clock, and one that simulates a DUT as depicted in figure (2.2b).

It's important to note that in a practical imaging application, a Michelson interferometer with a circulator is used to create an interference pattern with an actual DUT. The system in figure (2.1) merely simulates the behavior of an actual system with the Mach-Zehnder interferometer as a DUT simulator, since a reflection measurement is not included.

After an optical to electrical conversion through two balanced receiver photodetectors, the interference patterns are applied to an A/D converter. The DUT interferometer pattern is sampled at each optical clock zero-crossing which produces a digitally sampled sinusoidal pattern as seen in figure (2.2d). An FFT is then applied to the sample waveform from figure (2.2d) which ideally exhibits a narrow impulse with high signal to noise ratio (SNR) as seen in figure (2.2e). Based on this frequency measurement and the theoretical calculations in the following section, a distance measurement can then be inferred. If a Mach-Zehnder interferometer is used as the DUT, then the calculated distance measurement will remain constant. Various distance profiles can be tested by utilizing different sized Mach-Zehnder interferometers. 


\section{1 - Frequency Modulated Continuous Wave (FMCW)}

The frequency modulated continuous wave implementation is achieved by creating a source that linearly increases in frequency over a set frequency bandwidth. The linearly increasing frequency is emitted into an optical splitter as seen in figure (2.1), which sends the same frequencies to the DUT and an optical combiner. By the time a signal reflects back from the DUT, it combines with a higher frequency transmitted signal. The transmitted signal and received signal frequency difference depends on the sweep rate. Figure (2.3) provides a visual representation for this implementation.

The transmitted signal and reflected signal can be summed to create a continuous pattern that can be used to calculate the distance to the reflection event.

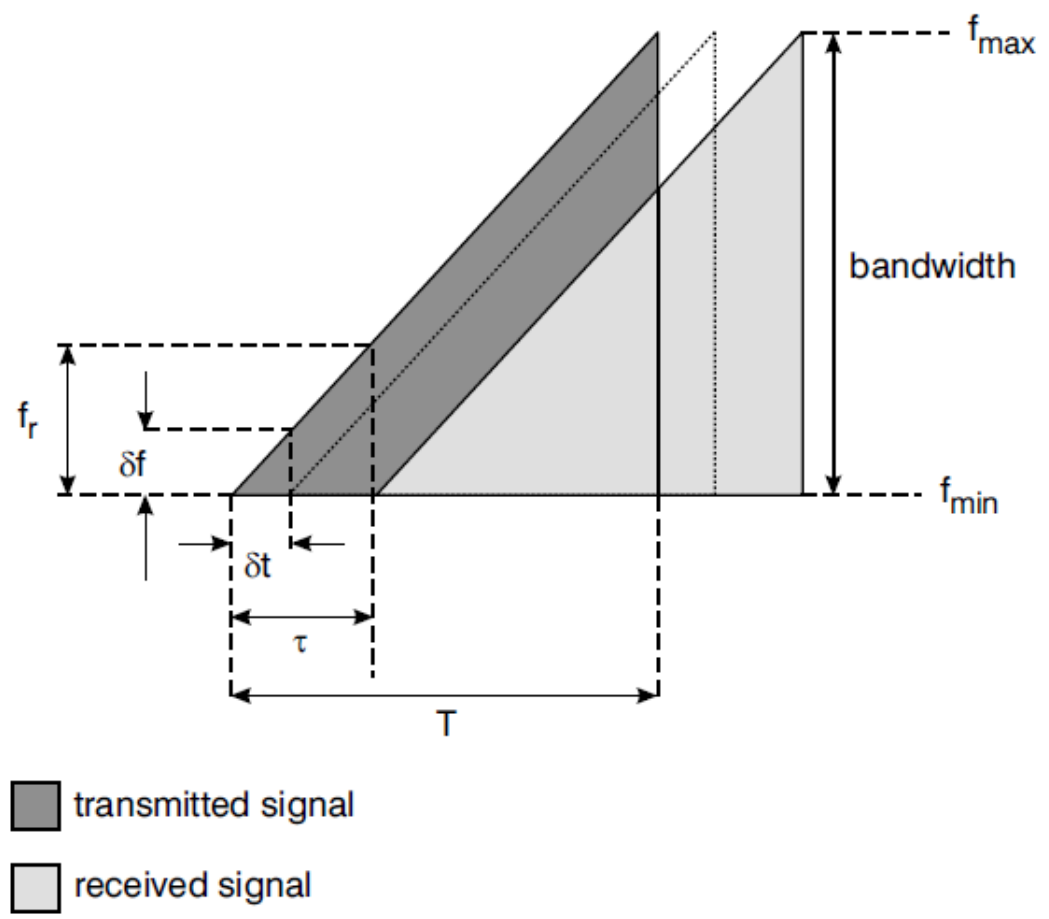

Figure 2.3: FMCW graph of transmitted and received signals from a stationary target [2] 
The FMCW architecture reveals information about the range of an object due to the relationship between the velocity of propagation $v$, round trip time delay $t$, and distance $d$ of the object [3]:

$$
v=\frac{2 d}{t}
$$

The velocity of propagation is a constant in the system that will be used to reveal critical distance information about the objects of interest. The frequency bandwidth, $B$ and sweep time $T$ contribute to the relationship between the transmitted and received signals. Equation (2.2) shows how these parameters provide the time delay $\tau$ :

$$
\tau=\frac{f_{r}}{\left(\frac{B}{T}\right)}
$$

Combining equation (2.1) and (2.2), the distance $d$ of the object can be found [3]:

$$
d=\frac{v}{2} \frac{f_{r}}{\left(\frac{B}{T}\right)}
$$

For systems with a large detection range, the change in the difference frequency will be very small, and possibly undetectable because of linewidth limitations on the transmitting source, for a very small change in the distance of the object [3]. This result is shown in equation (2.4) by solving for the measured frequency:

$$
f_{r}=\frac{2 d}{v} \frac{B}{T}
$$

As the sweep time decreases, while the other parameters remain constant, the measured frequency difference increases. Thus, as the sweep rate becomes faster, the frequency sensitivity improves. 
Another factor that contributes to the precision of measurement is the frequency bandwidth, $B$. From figure (2.3), the smallest detectable time measurement can be related to the inverse of the sweep range. Equation (2.5) demonstrates how the range $R$ is related to the time delay:

$$
R=\frac{c \tau}{2}
$$

By differentiating both sides and substituting the inverse of the frequency bandwidth $(1 / B)$ for the time delay $\tau$, equation (2.6) reveals the minimum detectable distance.

$$
\delta R=\frac{c \delta \tau}{2}=\frac{c}{2 B}
$$

This equation reveals that as the frequency bandwidth of the FMCW waveform increases, the range resolution becomes smaller, which translates into a more precise measurement of the range of an object [2]. Throughout chapter 5, an increased effort will be made to increase the frequency bandwidth of the tunable laser to allow for precise distance measurements. 


\section{2 - OCT Medical Imaging Applications}

A full appreciation for OCT can only be achieved with an overview of the various medical applications that this imaging technique provides. OCT is known for creating extremely high resolution images in real-time that offer many advantages over competing imaging schemes. For example, MRI (magnetic resonance imaging) requires a stationary enclosed setting which is uncomfortable, most notably due to acoustic noise effects. The acoustic noise is caused by changes in the Lorentz force experienced by the gradient coils, which create expansions and contractions within an audible frequency range with a sound intensity up to $120 \mathrm{~dB}$ (A) [4]. OCT measurements don't cause any discomfort and can be made portable, which has important implications that will be discussed in depth in this section.

Another advantage is the use of non-ionizing radiation. X-ray imaging uses much higher frequency radiation which can cause the electrons in atoms to dislodge themselves from their stationary orbital paths. Enough energy can remove an electron entirely; hence, X-rays are classified as having possible ionizing radiation effects. A more detailed explanation of discrete electron energy levels and their corresponding frequencies will be presented in chapter 3 , which will make this OCT advantage more apparent and better understood.

The most important advantage that OCT has over other imaging techniques is evident by comparing it with ultrasound images. Ultrasound can penetrate deep within organic material, is portable, and doesn't contain ionizing radiation; however, it lacks the high resolution quality that OCT can obtain. OCT can nearly detect images down to the cellular level, which allows for more efficient diagnoses, and provides a better insight 
into inner organic structures. Specific situations call for the different image sensing procedures listed above, but when a high resolution image is required, OCT fulfills that objective. The remainder of chapter 2 justifies this statement through its implications in medical applications including: cardiovascular medicine, the musculoskeletal system, oncology, neurosurgery, and nerve repair.

\subsection{1 - Cardiovascular Medicine}

Cardiovascular disease represents the leading cause of death worldwide and is among the most important applications of OCT [5]. There are many OCT applications in cardiovascular medicine, particularly concerning acute coronary syndromes (ACS) and strokes, which will be discussed. Among these applications, the most important is the diagnosis and treatment of ACS and its precursor, coronary artery disease [5].
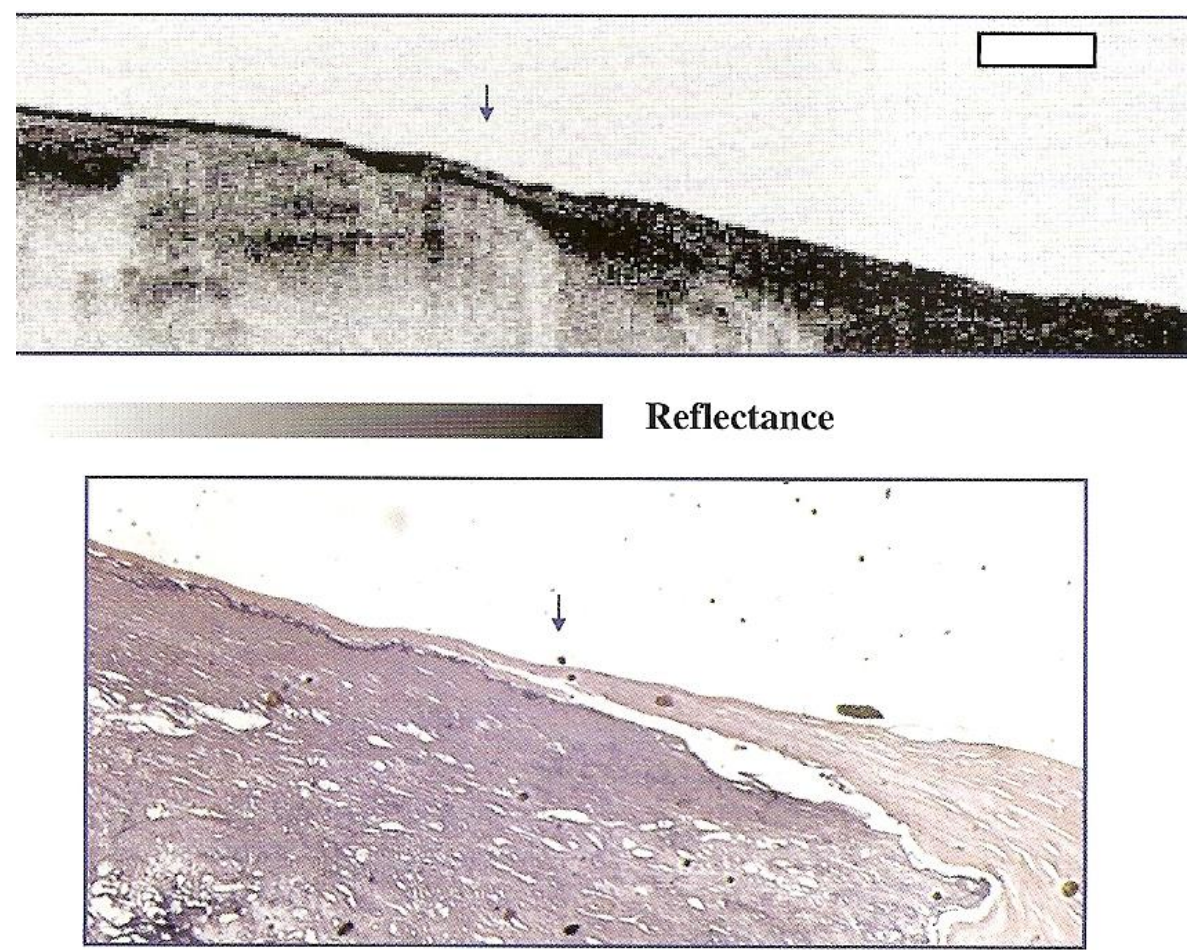

Figure 2.4: OCT image of heavily calcified plaque with corresponding histopathology [5] 
Coronary artery disease, or coronary heart disease, is a condition where plaque builds up within the inner artery walls and prevents blood flow to the heart. As plaque collects, also known as atherosclerosis, oxygen-rich blood can't reach the heart which may result in a heart attack. Early detection of this plaque helps prevent subsequent build-up and may ultimately save lives. Figure (2.4) demonstrates how OCT is used to detect early plaque build-ups. The corresponding histopathology (typically a medical biopsy) is presented as a frame of reference.

Interventional OCT cardiology is typically performed with a catheter based approach. A stent, depicted in figure (2.5), attached to a catheter is placed within the artery while the walls are being continuously imaged for plaque. Once the high-density plaque build-up is observed, the stent is expanded which compresses the plaque, widens the artery, and increases blood flow.

OCT also has the potential to both identify plaque that leads to a stroke and guide to interventional procedures in the cerebral circulation [5]. A stroke is the lack of brain activity due to an instable amount of blood flow. The assessment and treatment of plaques help in preventing strokes in the same way as preventing coronary artery disease.

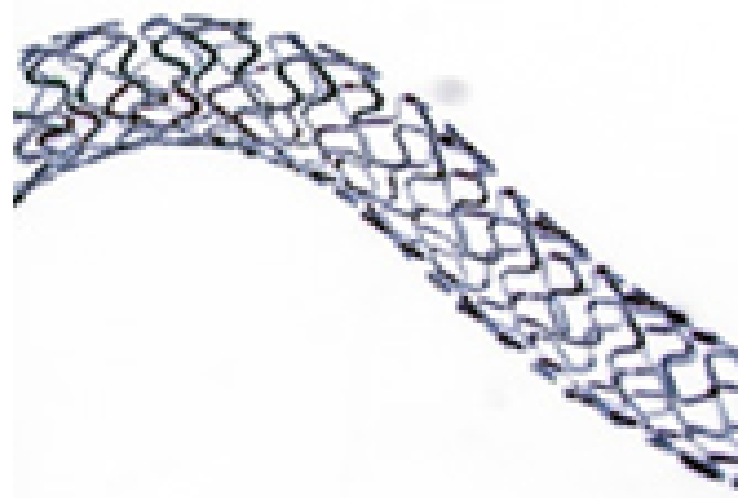

Figure 2.5: Stent used in interventional OCT cardiology 


\subsection{2 - Musculoskeletal System}

There are a large number of potential applications for OCT in the musculoskeletal system, but likely the most important are in the early diagnosis and monitoring of osteoarthritis [5]. The disorder mainly affects the knee and hip, which can be diagnosed through morphologic changes, such as cartilage degeneration. Collagen makes up 50\% of the dry weight of cartilage. It is a fibrous protein that has a rod-like shape and is insoluble in water [6]. Through the imaging assessment of the cartilage water content, osteoarthritis (OA) can become clinically evident. OCT imaging helps in confirming that the edges of tissue contain no significant OA. In particular, micropenetrations are actually through the subchondral plate, and the development of new cartilage (through cartilage repair) is monitored [5].

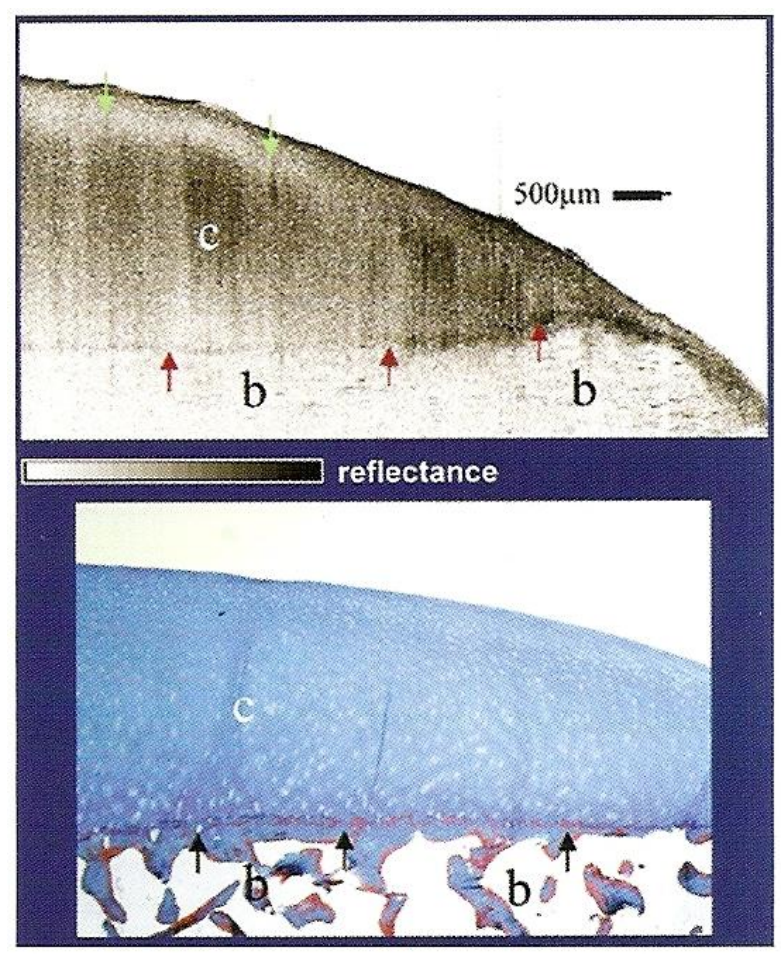

Figure 2.6: OCT image of normal human cartilage. The top is the OCT image, the bottom is the histopathology. Cartilage (c) and bone (b) can be clearly identified in vitro along with the bone cartilage interface [5]. 


\subsection{3 - Oncology}

The identification of early malignant changes of cancer is the central objective of clinical medicine. If the onset of cancer is detected too late in tumor growth, most become incurable. OCT plays a role in assessing tumor margins during surgical resection, the efficacy of therapy in promoting tumor death, and of blocks of tissue being assessed by pathologic processing [5]. OCT imaging can show its greatest contributions to oncology through the early detection of epithelial cancers.

Epithelium is a primary body tissue that contains cells that line the cavities and surfaces of structures throughout the body. Typically, epithelia are classified by four parameters: the number of cell layers, the shape of the component cells, the presence of surface specialization, and the presence of glands. To detect early malignancy (tendency for tumors to become progressively worse), among the tissue properties which OCT could potentially measure include width of the epithelium, nuclear size, nuclear variation, concentration of mitotic figures, integrity of the basement membrane, nuclear to cytoplasmic ratio, organization of glands, and presence of abnormally superficial nucleated cells [5]. Current OCT standards lack the resolution quality to detect many of these factors.

OCT also has a small role in the screening for cervical cancer. It will help assess patients with positive Pap smears, which will focus on intraepithelial neoplasia. Cervical intraepithelial neoplasia is the potentially premalignant transformation and abnormal growth of cells on the surface of the cervix [7]. OCT provides a potential solution to detecting early malignancies of many forms of cancer, but this high resolution realization is challenging. 


\subsection{4 - Neurosurgery}

Similarly to oncology applications, the role OCT will play in neurosurgery is dependent on future higher resolution technologies, but potential applications will be discussed. OCT has the potential to guide tumor resection on a micron scale, but is limited by the penetration depth [5]. It becomes difficult to image depths further than a few millimeters; therefore, techniques would be implemented to image particular points during brain surgery to assess margins. In neurosurgery an increased effort is made to avoid damaging neurological pathways. OCT has the potential for assisting in neurosurgery, which will help reduce this unfortunate and sometimes unavoidable surgical risk. Likewise, OCT also has the potential in aiding spinal surgery by reducing nerve injury.
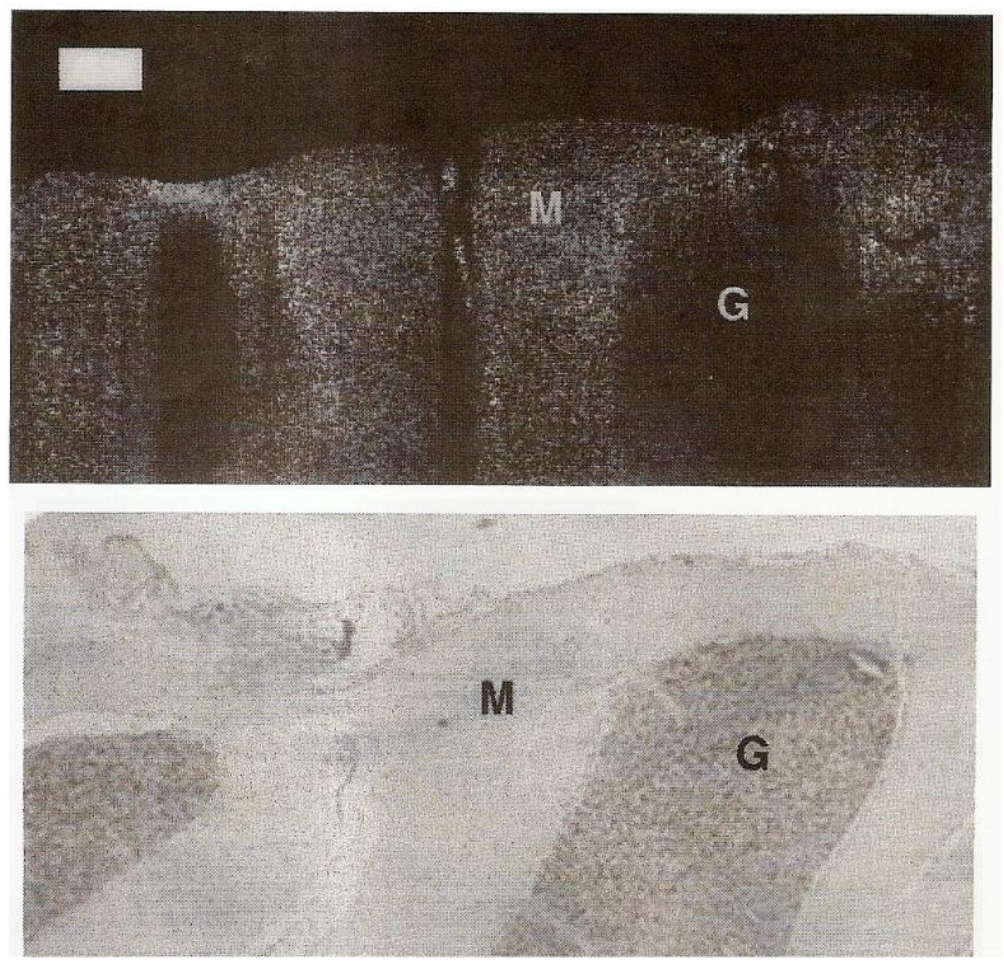

Figure 2.7: OCT image of human cerebellum. The grey matter $(M)$ and white matter (granular) (G) are identified in the OCT image and its corresponding histopathology [5]. 


\subsection{5 - Nerve Repair}

OCT has the potential of improving the outcome of surgery to repair transected nerves, while imaging through the nerve and distinguishing motor from sensory nerves [5]. Nerves are grouped together in fascicles, which are bundles of nerve fibers. These nerve fibers can become damaged during a trauma but are amenable to repair. The two predominate methods of repairing injured nerves are epineural (with or without interfascicular stitches) and perineural (fascicular suturing) [8]. The problem with these options is that it's difficult to assess the position of fascicles because the nerve covering is not easy to see. OCT provides a solution to this problem, which is evident in figure (2.8), where (f) are fascicles and (b) is bifurcation (splitting of nerves).
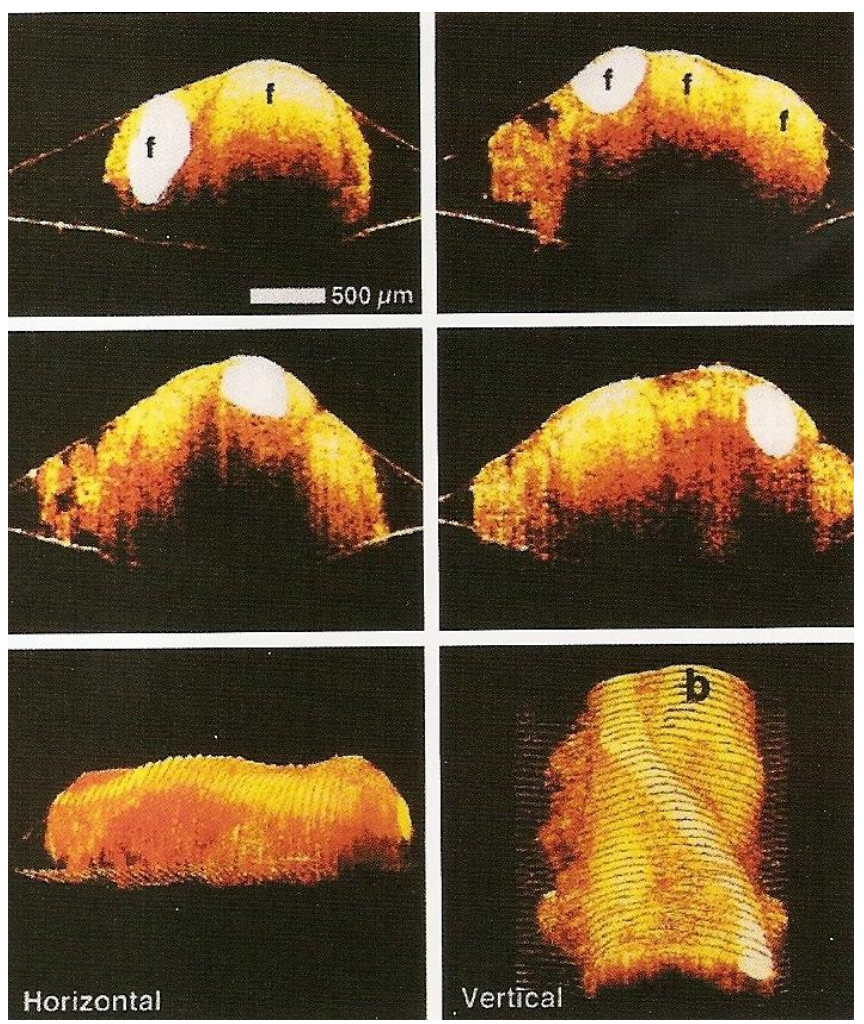

Figure 2.8: Three dimensional OCT image of a nerve [5] 


\section{CHAPTER 3 - SG-DBR Tunable Laser}

\section{1 - Laser Physics}

The word laser is an acronym for Light Amplification by Stimulated Emission of Radiation [9]. For lasing to occur, certain physical requirements must be satisfied. The process begins with stimulated emission of photons within the amplifying medium. The photons released from an exchange of energy, that will later be discussed, are incoherent and scattered. In many ways, this step resembles light emitted from a light bulb, almost an ideal isotropic electromagnetic radiation pattern. Lasers, however, are spatially coherent which is accomplished with the use of two optical feedback mirrors that create directionality and the necessary gain for true lasing. Spontaneous and stimulated emission is constantly occurring, which continually provides photons for the loss created by the partially transmitting mirror. Another subtle but important requirement for lasing is an appropriate distance between the resonating mirrors. The round trip reflected phase change must be such that it doesn't create a destructive interference pattern, essentially cancelling out the wave.

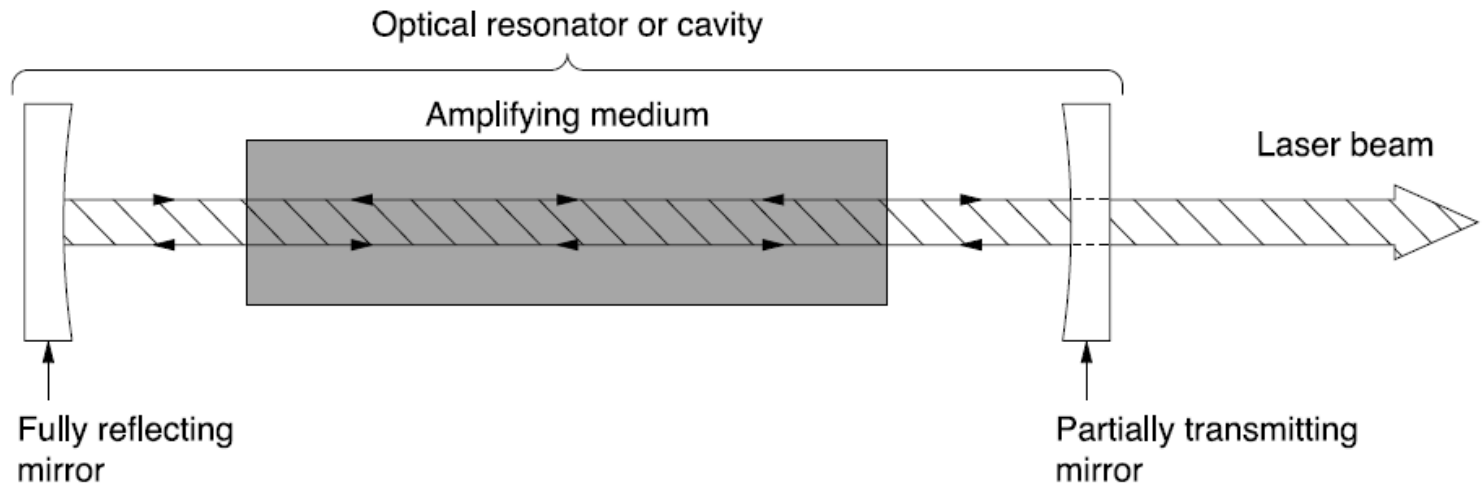

Figure 3.1: General schematic of typical laser operation [9] 
The general functionality of a laser is quite simple from a conceptual point of view, but a deeper understanding of the physics is necessary to manipulate its functions for the image sensing applications previously discussed. In particular, the exchange of energy between electrons in an atomic structure and how injected photons cause stimulated emission will be explained.

Inside an atom, electrons are restricted to discrete energy levels that orbit the nucleus. The quantum nature of matter precludes electron energies from existing on a continuous energy distribution. Although this seems like a limiting physical feature, this property leads to many useful applications, such as the photoelectric effect and particularly laser operation. Wave-particle duality suggests that light travels in discrete energy packets called photons with a specific frequency of propagation. This photon energy and frequency is related by the equation below where Plank's constant, $h=6.626 \times 10^{-34} \mathrm{~m}^{2} \mathrm{~kg} / \mathrm{s}$.

$$
E=h f
$$

As electrons orbit at specific energy levels, they can only absorb frequencies of light predicted by equation (3.1). The more tightly packed electrons, closer to the center, require a higher amount of energy to essentially dislodge them from their position to a lower energy state, further from the center. This process, known as stimulated absorption, is critical in fully understanding optical amplification. The Pauli Exclusion Principle states that only one electron can occupy one quantum state at a given time, which leads to processes called stimulated emission and spontaneous emission. 
Not all of the electrons in an atom can exist in the first orbital path, which results in a population inversion. These electrons in excited energy states can spontaneously emit specific wavelengths of light as they decay down to a ground state [10]. This random process, related by the half-life of the electrons, can also be caused by a directed photon that stimulates the emission. Unlike stimulated absorption, the electron-photon interaction causes electron movement back to the initial ground state with a new copy of the initial photon in addition to the original photon. This concept is the fundamental process for light amplification and lies at the core of laser functionality. Figure (3.2) shows the newly created photon caused by an electron recombining with the ground state.

As previously discussed, this light is then continuously reflected and amplified by the use of two mirrors to create a coherent optical source, known as a laser. Now that general lasing operation has been explained, a detailed explanation of laser diodes is necessary to fully understand the tunable SG-DBR laser.

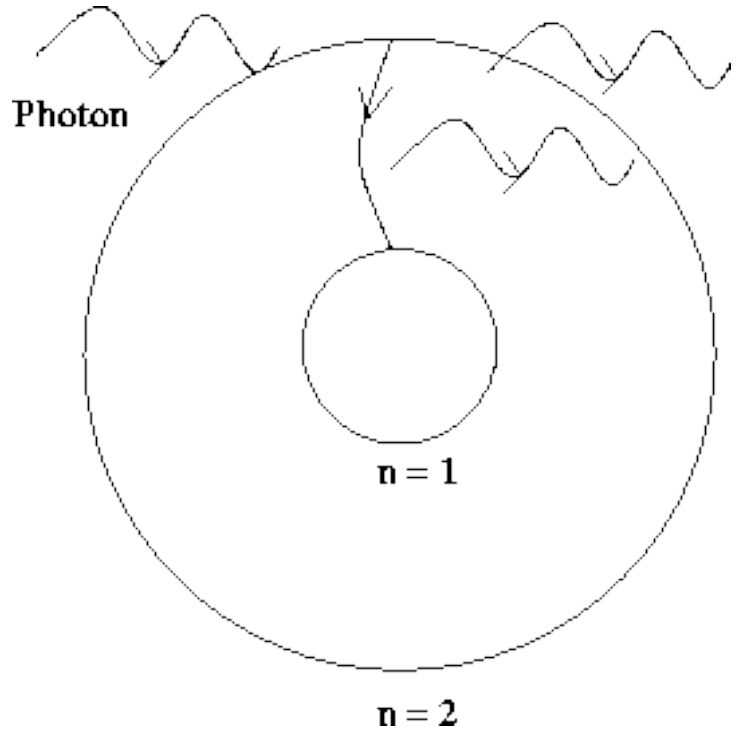

Figure 3.2: Displays the stimulated emission caused by the electron movement from an excited state to the ground level 


\section{2 - Semiconductor Laser Diode Operation}

Similar to a typical diode, semiconductor laser diodes function through the use of a p-n junction. As a forward biased electric field is applied to the p-n junction, electrons begin to drift across the depletion zone and recombine with the holes. Laser diodes contain a band overlap that creates interband transitions to be radiative. A laser diode is so heavily doped that the Fermi energy (energy of a highest occupied quantum state that exists in a system) appears to be in the p-valence band and the n-conductive band. In a solid crystal lattice, lower energy bands are filled first and are continually filled as the energy increases, while still satisfying the Pauli Exclusion Principle. If the highest occupied level is inside a band, the energy of this state is called the Fermi level. That band is therefore only partially filled. The Fermi level thus lies between the highest energy fully filled band (called the valence band) and the lowest energy empty band (called the conduction band) [11]. The contact potential shift is great enough that this overlap recombination energy will emit photons of the same wavelength which will continually stimulate more transitions [12].

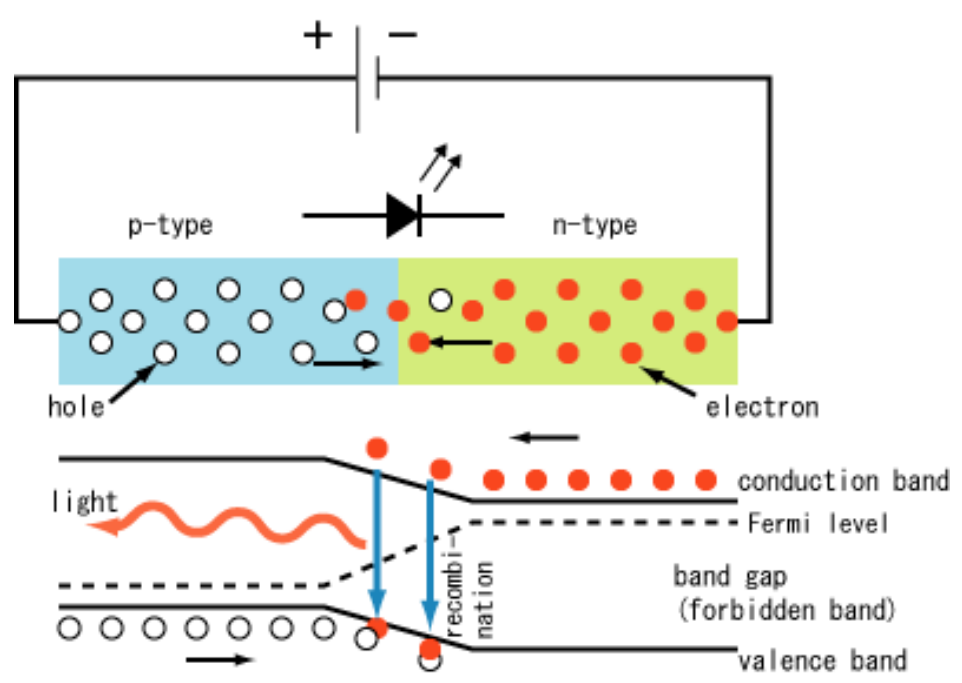

Figure 3.3: Energy band diagram of recombination transition across Fermi level [13] 


\section{3 - Structure of SG-DBR Laser}

The SG-DBR laser is operated through the control of five different inputs. Similar to a general laser diode, the SG-DBR laser emits photons by a range of discrete electron recombination energies in the gain section. Each section contains a forwardbiased p-n junction diode that all affect the laser differently. The gain section turns the laser on and provides an amplification region for continued lasing. The semiconductor optical amplifier (SOA) allows for the necessary power leveling of the output signal. The front, back, and phase mirrors control the wavelength tuning functionality.

The front and back mirror sections of the laser consist of Sampled GratingDistributed Bragg Reflectors, which provide coarse wavelength tuning across the tuning range of the laser. The phase section provides an extremely narrow range of continuous wavelength tuning. Combining the capabilities of all three tuning sections, the laser can be tuned continuously to any wavelength within the operating range of the laser [3]. As previously discussed in chapter 2, continuous linear wavelength tuning is critical for optimum OCT measurements.

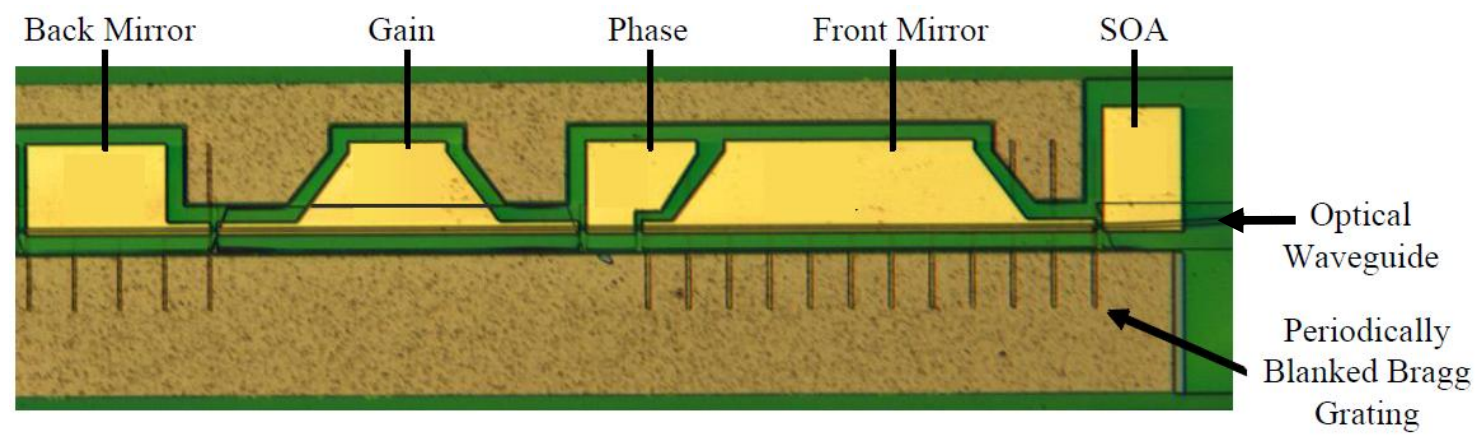

Figure 3.4: Top view of the laser showing the five primary sections [3] 
The various band gap regions in figure (3.5) represent the allowed paths of the light. Low band gap regions provide light amplification by causing electrons to recombine with holes in the valence band. High band gap regions provide low-loss transmission of photons through the area. It's important to note that both the gain and SOA sections contain low band gaps; this causes the necessary stimulated emission for either lasing operating sustainability or increased optical power.

Figure (3.6) illustrates the periodically blanked Bragg gratings which impose various reflectivities between the front and back mirrors. The mirrors exhibit slightly different periods in which the Bragg grating is blanked as demonstrated by the distances $\mathrm{L}_{1}$ and $\mathrm{L}_{2}$ in figure (3.6). This results in a different reflectivity peak spacing for the front mirror and back mirror tuning sections, since the reflectivity peak spacing is a function of the distances $\mathrm{L}_{1}$ and $\mathrm{L}_{2}$ [14]. For the JDSU laser, the reflectivity peak spacing is approximately $5 \mathrm{~nm}$ for the front and back mirror sections [3]. The small difference in reflectivity peak spacing ensures that only one set of reflectance peaks is aligned at any given time [14].

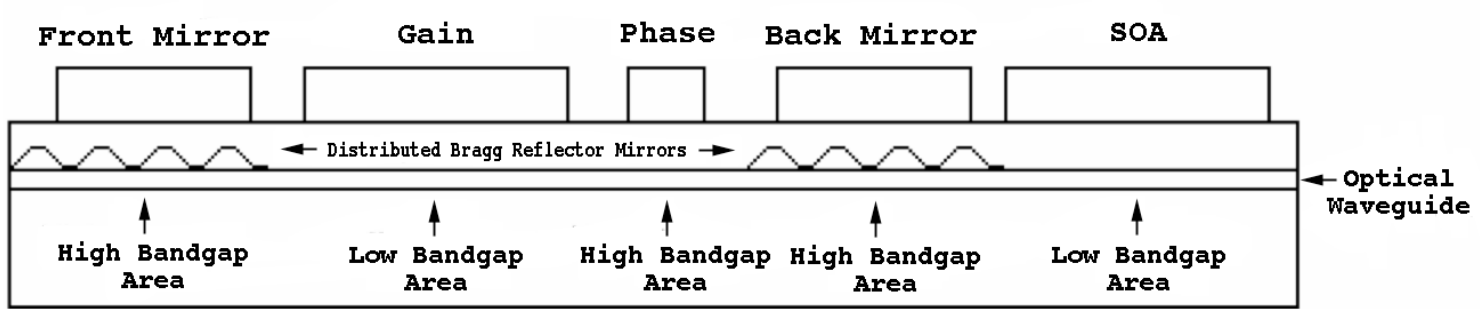

Figure 3.5: Cross section of the laser showing the various band gap areas [15] 
Back Mirror
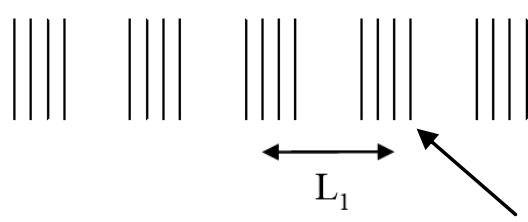

Bragg Reflectors
Front Mirror
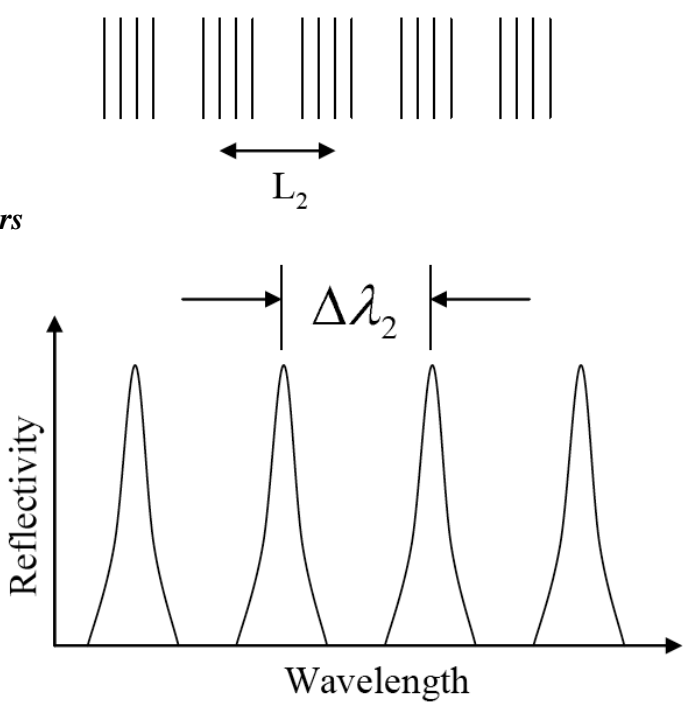

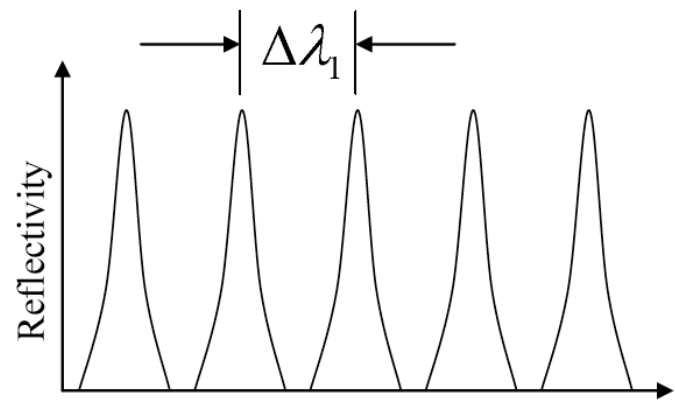

Wavelength

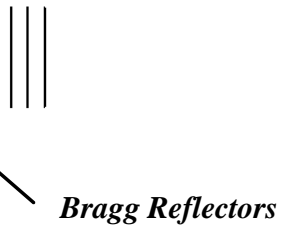

Wavelength

Figure 3.6: Bragg reflectors and their reflectivity for the front mirror and back mirror. The spacing differences, $L_{1}$ and $L_{2}$, are related to the wavelength differences, $\Delta \lambda_{1}$ and $\Delta \lambda_{2}$, by equation (3.2) and (3.3) below, where $\mathrm{n}$ is the refractivity index [3].

$$
\begin{aligned}
\Delta \lambda_{1} & =\frac{\lambda^{2}}{2 \mathrm{~nL}_{1}} \\
\Delta \lambda_{2} & =\frac{\lambda^{2}}{2 n L_{2}}
\end{aligned}
$$




\section{4 - System Block Diagram}

The five sections of the SG-DBR laser are all connected with the corresponding inputs and output as shown in figure (3.7). Agilent 33220A arbitrary function generators are used to control the front mirror, back mirror, phase mirror, and SOA sections of the laser. These are necessary to provide synchronized voltage waveforms, which allow for continuous frequency tuning. A D.C. current source is used to control the gain section, which causes stimulated emission within the laser. The voltage outputs of all five control sections are converted to current inputs to the laser through the use of current limiting resistors [3]. An LDC-3744 B thermoelectric cooler is used as a temperature feedback control to keep the internal laser temperature constant. The laser output is emitted into an optical isolator which prevents against optical reflections. The optical output is then analyzed with an Agilent 86140B optical spectrum analyzer.

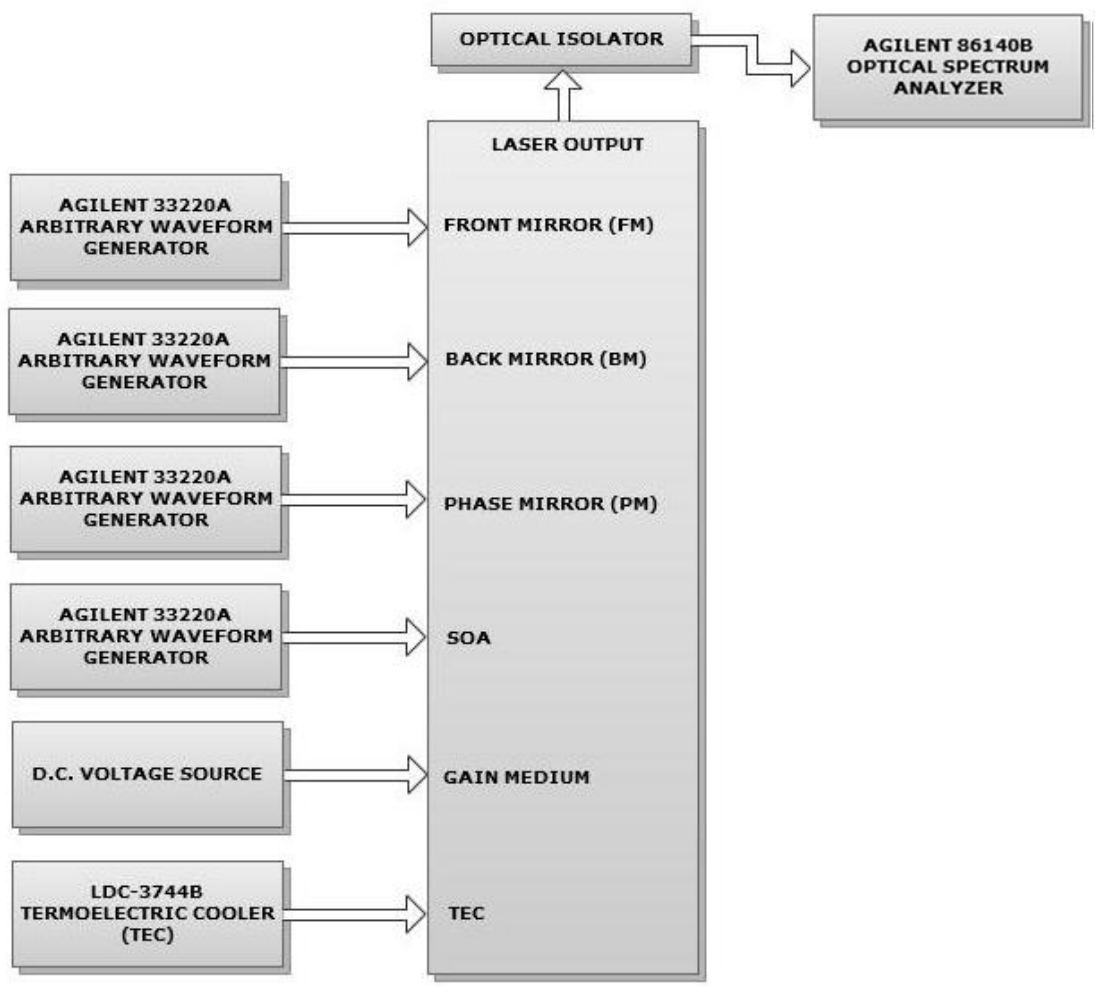

Figure 3.7: Block diagram of SG-DBR laser with corresponding inputs and outputs 


\subsection{1 - Laser Package Assembly}

The JDS Uniphase SG-DBR laser is packaged in a 24-pin butterfly package. The laser has intrinsic low capacitive inputs, which need to be carefully connected to external drive sources for high speed frequency ramp generation. To minimize degeneration of the arbitrary waveform generator signals, semi-rigid coaxial cables are used to interface with the butterfly inputs to the laser. These provide a $50 \Omega$ environment and enable the potential application for high frequency drive currents to the tuning sections of the laser [3]. Another packaging consideration involves a metallic base used as a heat sink. The thermoelectric cooler (TEC) controls the temperature of the laser, which is continually set to $22^{\circ}$ Celsius. The TEC doesn't require high frequency currents, so semi-rigid cables aren't necessary for this operation.

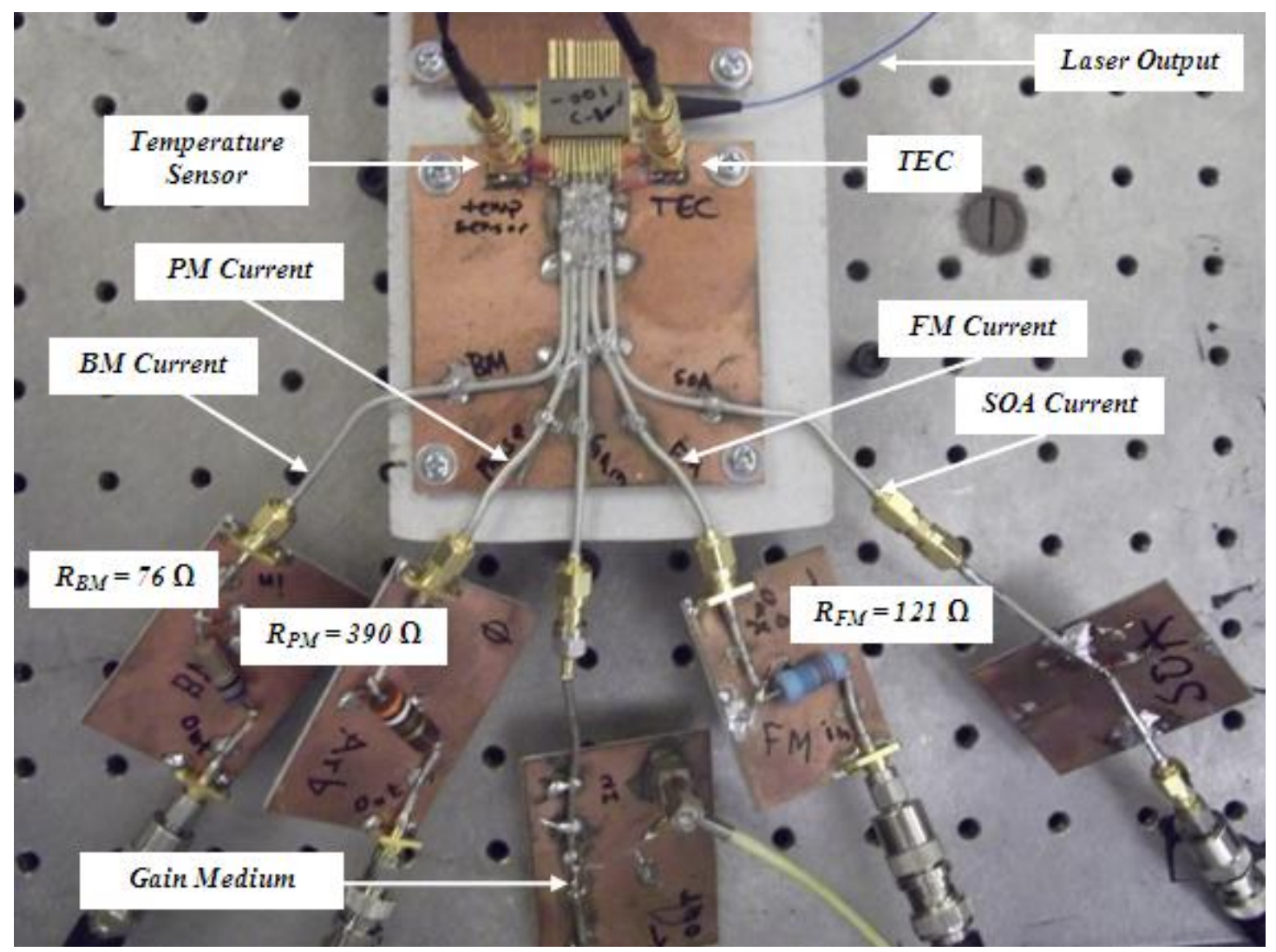

Figure 3.8: Laser Assembly 
Once the laser is properly configured, the laser output is fed into a fiber pigtail. This is then coupled to an optical isolator, which is shown in figure (3.9). The isolator blocks any reflections created in the system, which prevent possible unwanted interference patterns at the output. Reflections are typically prevented through the use of a device called a Faraday rotator. The light is initially vertically polarized which is then rotated by $45^{\circ}$ through the Faraday Effect. The output contains a $45^{\circ}$ polarized analyzer that allows a full transmission of light. Light travelling in the backward direction also becomes polarized at $45^{\circ}$ by the analyzer, but is rotated in the same direction regardless of propagation. At this point, the light has become horizontally polarized and since the input polarizer is vertically aligned, the light will be extinguished [16]. Faraday rotators used in the optical communication bands are typically made out of yttrium iron garnet (YIG) crystals. As stated before, the output of the isolator is then fed into an optical spectrum analyzer for wavelength analysis.

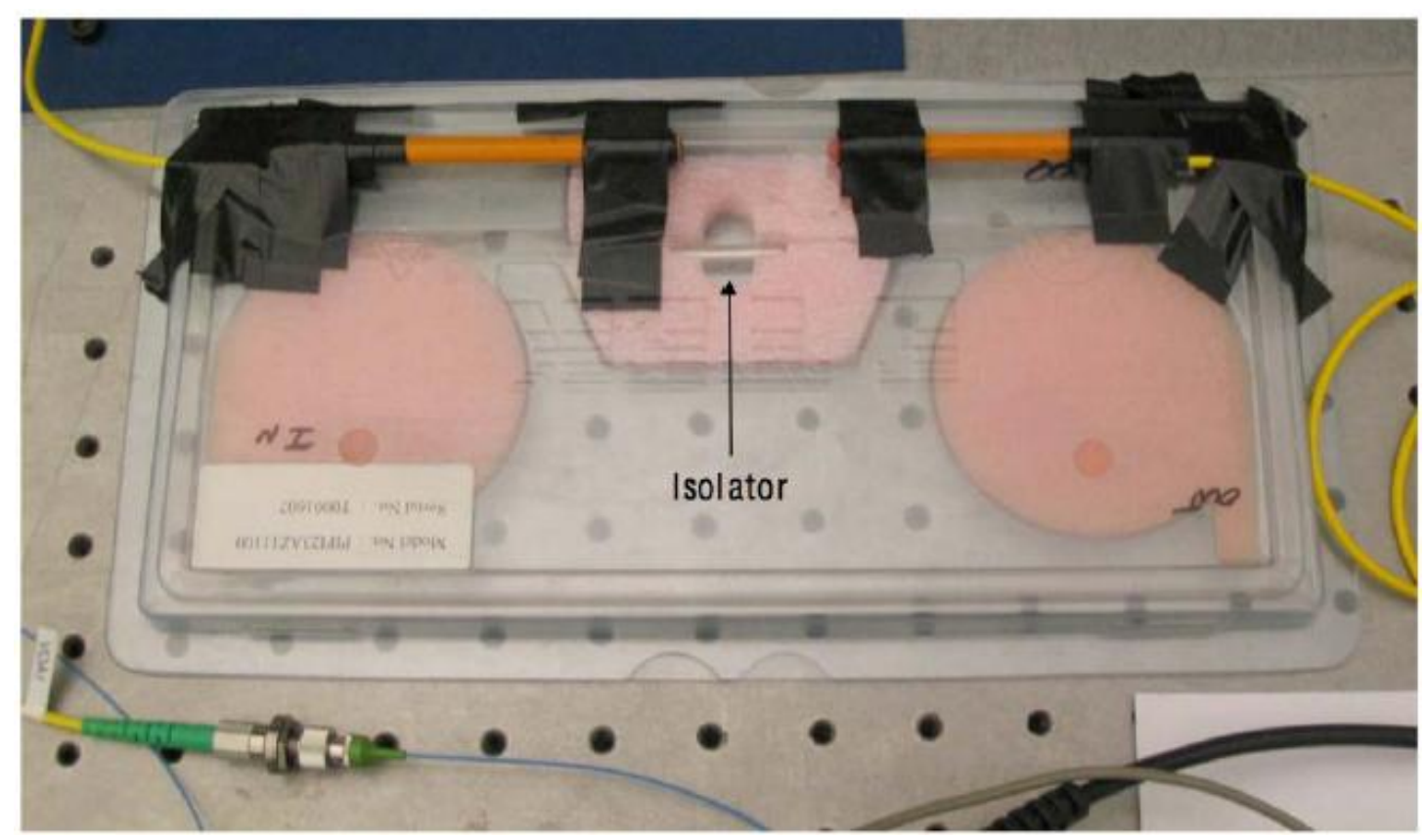

Figure 3.9: Optical isolator [15] 


\section{5 - Single Mirror Tuning}

As previously discussed, the tuning mirrors consist of diodes that can change the refractive index within the grating. This is accomplished by simply altering the forward bias current injection that is applied to each diode section [3]. The average waveguide index of refraction is approximately 3.3 [17]. Various currents can alter the reflectivity peaks which influence the laser frequency. By adjusting a single mirror at a time, the reflectivity peaks slowly shift into a new mode. As the peaks shift, weaker side mirror modes become apparent. Once a peak is shifted by enough injection current, the weaker wavelength mode takes over. This single mirror tuning allows for $5 \mathrm{~nm}$ increments which are evident in figures (3.10) and (3.11).
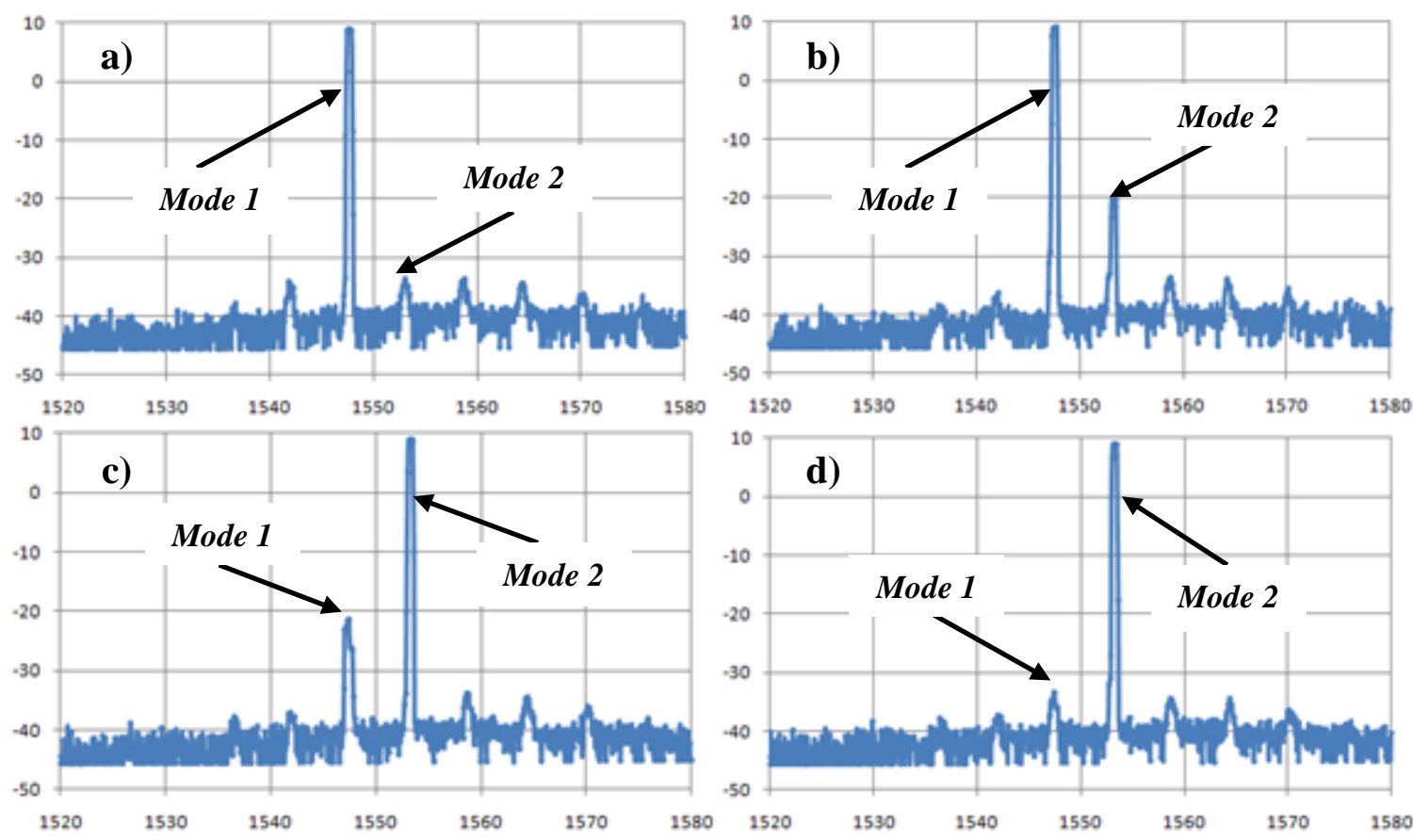

Figure 3.10: Image a) demonstrates a single lasing mode with approximately $40 \mathrm{~dB}$ of side mode suppression. Images b) and c) show the transition from the lasing mode shown in image a) to another that is $5 \mathrm{~nm}$ higher in wavelength. Image d) shows the completed mode hop. 
Figure (3.11a) demonstrates the reflectivity spacing versus wavelength for the front mirror bias. When the reflectivity peak aligns with the back mirror bias reflectivities in figure (3.11c), a specific wavelength is generated as seen in figure (3.11e). As current is injected into the front mirror bias, the reflectivity peaks shift as seen in figure (3.11b). These peaks will now align with a back mirror bias peak at a $5 \mathrm{~nm}$ spacing depicted in figure (3.11d). Figure (3.11e) shows the different wavelength that the laser outputs. Simultaneous front and back mirror tuning is implemented similarly as seen in a qualitative depiction in figure (3.12) in the following section.
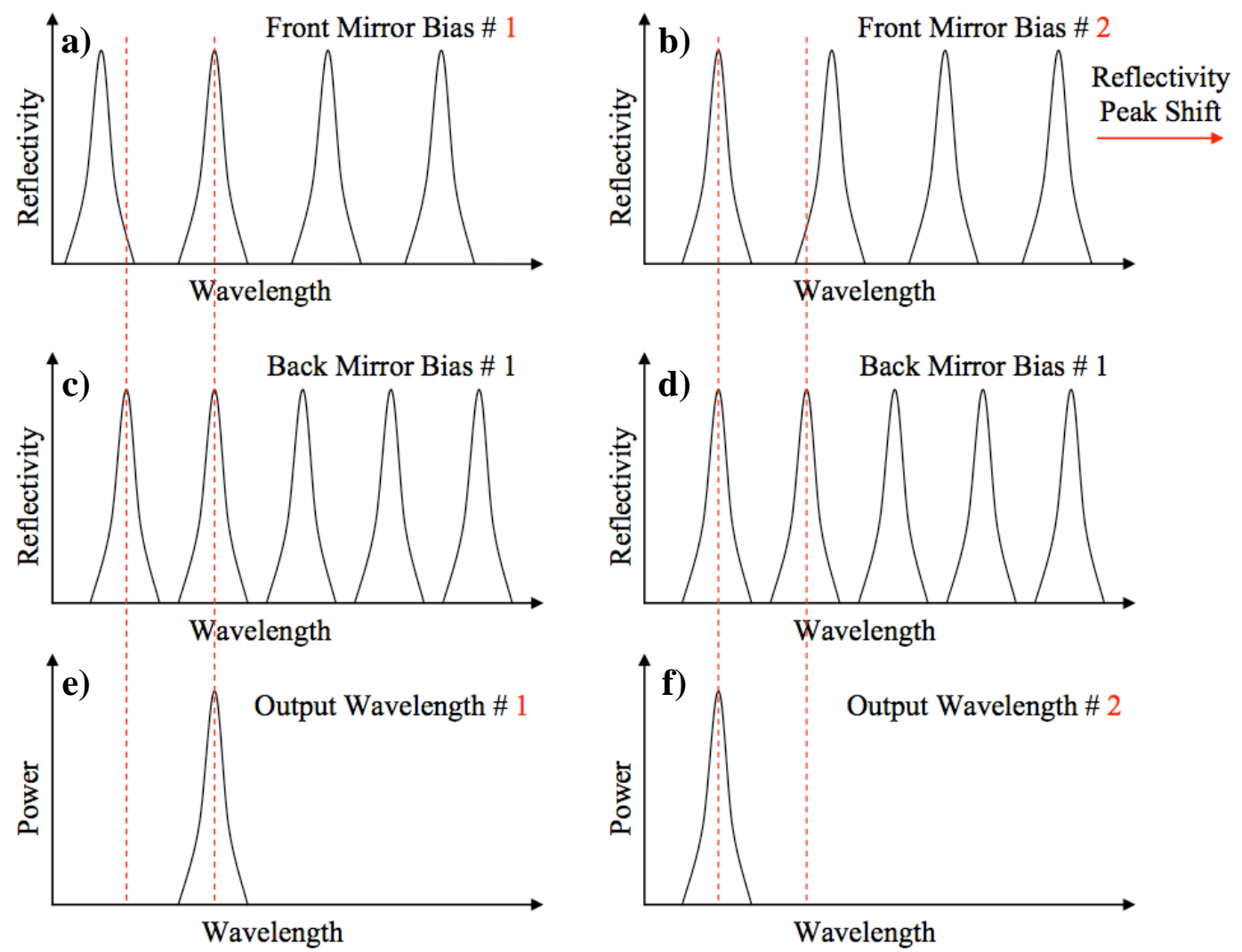

Figure 3.11: Qualitative demonstration of changing the bias current to the front mirror diode, which results in $5 \mathrm{~nm}$ mode hops [3] 


\section{6 - Simultaneous Front and Back Mirror Tuning}

Wavelength tuning becomes more continuous as current is injected into both the front and back mirror diodes simultaneously. Since the reflectivity peaks for each mirror are spaced at slightly different distances, as both peaks shift, wavelength modes occur more often. The change in refractive index results in a change in the velocity of propagation within the optical waveguide. This causes the shift in both reflectivity peaks, resulting in a small change in the wavelength, which corresponds to the cavity mode spacing [3]. As opposed to single mode tuning, simultaneous tuning to both Bragg reflectors create $0.3 \mathrm{~nm}$ increments. The side mode suppression analysis of twodimensional simultaneous mirror tuning is also provided in subsection (3.8.2).
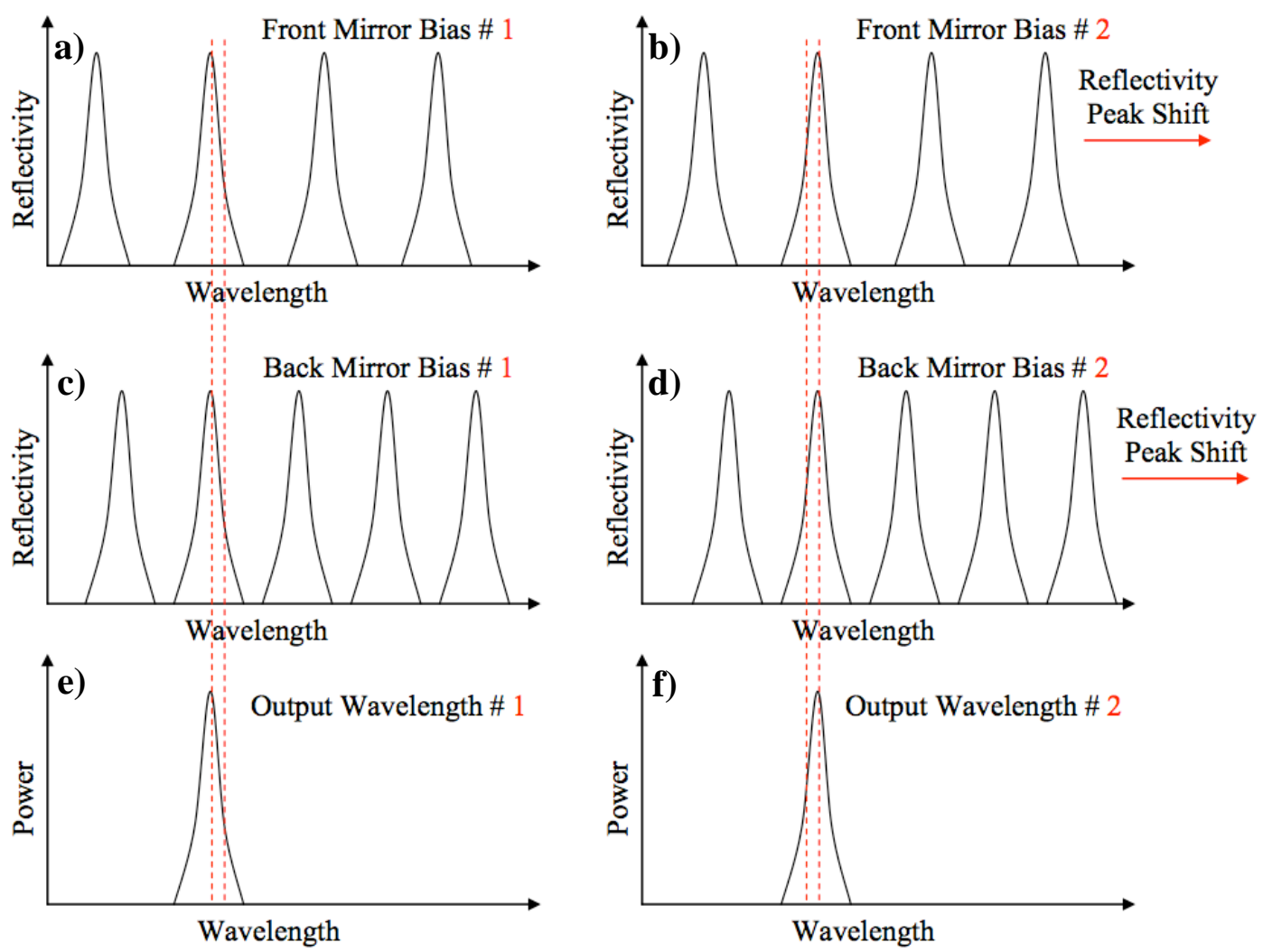

Figure 3.12: Qualitative demonstration of changing the bias current to the front and back mirror diode, which results in $0.3 \mathrm{~nm}$ mode hops [3]. 


\section{7 - Phase Mirror Tuning}

Although two mirrors allow for semi-continuous tuning, the incorporation of a third mirror is necessary for the desired continuous frequency ramps that are required for SS-OCT. Simultaneously tuning the current to both the front and back mirror sections produces continuous wavelength shifts until a $0.3 \mathrm{~nm}$ mode hop occurs [3]. The phase section provides an additional continuous $0.2 \mathrm{~nm}$ wavelength tuning range [3]. Tuning all three mirror sections synchronously creates a full wavelength tuning range without the instantaneous wavelength mode hops. Three-dimensional mirror tuning will be explored more in chapters 4 and 5 to create an automated laser calibration, where figure (3.13) depicts the $0.2 \mathrm{~nm}$ continuous phase mirror range.
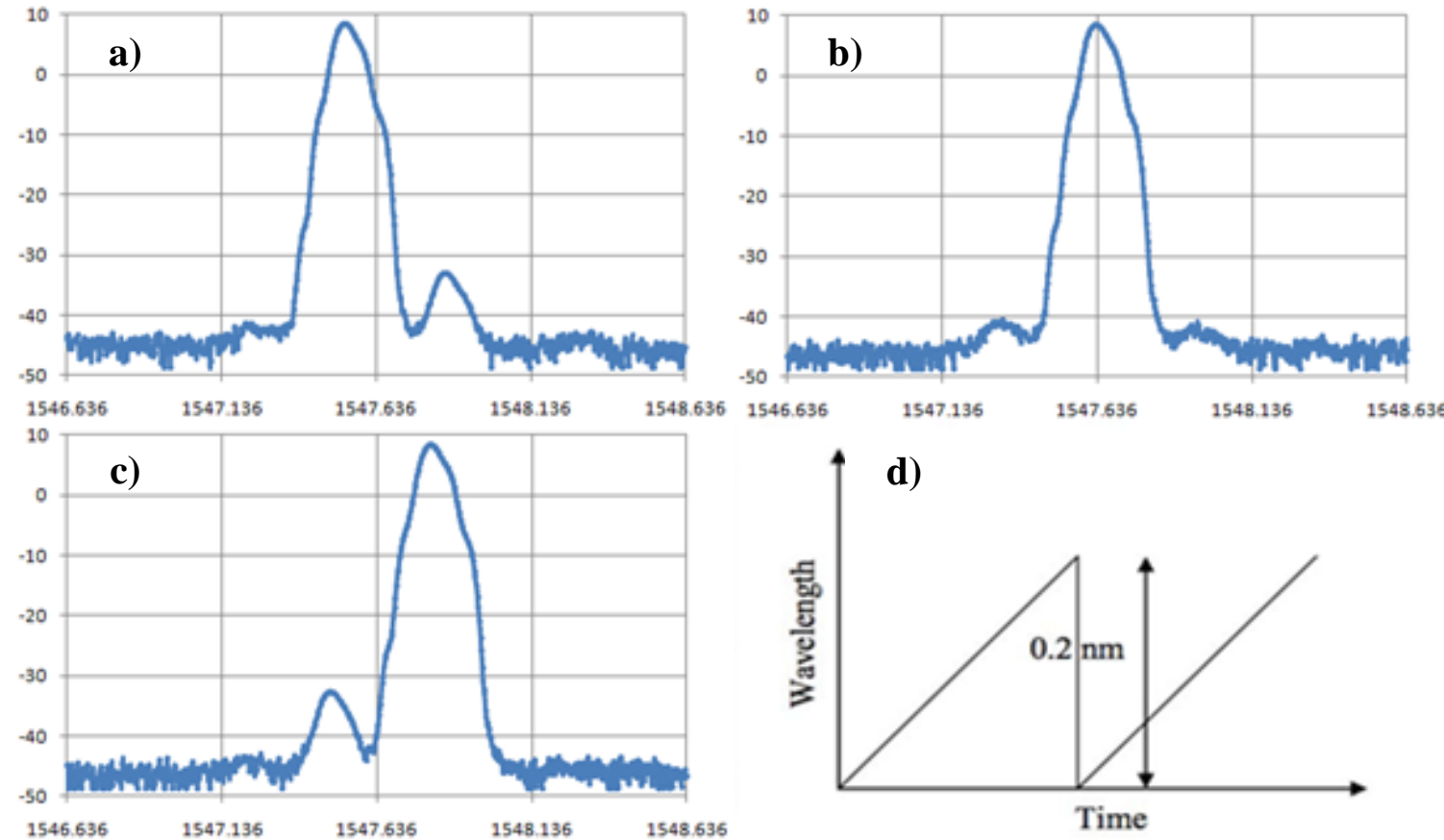

Figure 3.13: Adjusting the current to the phase section of the laser allows for a small but continuous range of wavelength tuning as seen through the progression of images a), b) and c). Image d) illustrates the $0.2 \mathrm{~nm}$ continuous tuning range. 


\section{8 - Various SG-DBR Laser Characteristics}

Now that the laser operation has been adequately described, specific intrinsic characteristics will be explored. The laser linewidth and continuous sweeping side mode suppression ratios were experimentally calculated and obtained through various techniques.

\subsection{1 - Laser Linewidth Characterization}

The linewidth of a laser, typically taken at the center frequency of a tunable laser, is the width of its optical spectrum at half of the maximum power in a power spectral density plot. The linewidth can be related to the temporal coherence, characterized by the coherence time or coherence length which arises from phase noise largely caused by $1 / \mathrm{f}$ frequency noise [18]. Another contributing factor arises from broadband noise generated at the gain medium. The coherence time and coherence length are inversely proportional to the linewidth by the equations below:

$$
\begin{gathered}
\Delta f=\frac{1}{\pi \tau_{c o h}} \\
L_{c o h}=v \tau_{c o h}=\frac{v}{\pi \Delta f} \\
v=\frac{2}{3} c .
\end{gathered}
$$

There are many mechanisms that influence the spectral widening of a laser, but a significant contribution of emission broadening can be explained through quantum mechanics. The uncertainty principle imposes constraints on how pure the emission frequency can be when an electron transistions to a lower energy state. The energy width $\Delta E$ of an energy level and its lifetime $\Delta t$ is related by equation (3.7). 


$$
\Delta E \cdot \Delta t \approx \frac{h}{2 \pi}
$$

Thus an energy level $u$ will have an uncertainty in energy $\Delta E_{u}$ associated with the uncertainty in the radiative lifetime $\tau_{u}[9]$.

$$
\Delta E_{u} \approx \frac{h}{\tau_{u} 2 \pi}
$$

This equation also applies to the lower energy level within the radiative transistion.

Therefore, equation (3.9) approximately represents the total effective energy width of a laser deduced by quantum mechanics.

$$
\Delta E_{T}=\Delta E_{u}+\Delta E_{l}
$$

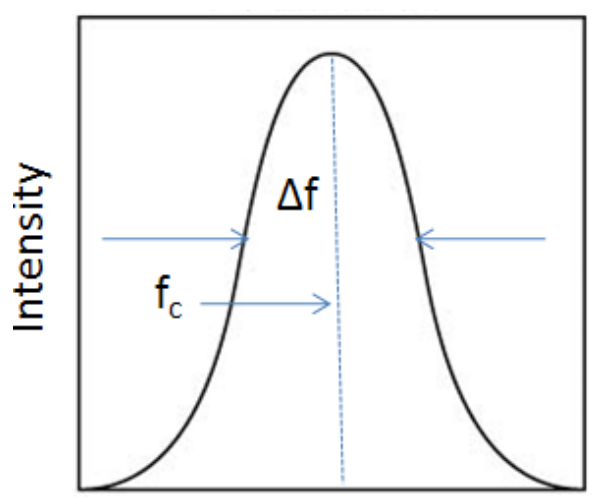

Frequency

Figure 3.14: General power spectral density plot that shows how the linewidth $(\Delta f)$ is qualitatively related to the center frequency $\left(f_{c}\right)$.

To calculate the linewidth, frequency fluctuations were converted into amplitude fluctuations, using a frequency discriminator. This was accomplished with two different length Mach-Zehnder interferometers. A Mach-Zehnder interferometer splits the incoming beam into two paths. The paths have a specific length difference which leads to a sinusoidal constructive and destructive interference pattern at the recombining 
output. The output can then be viewed as an amplitude modulated signal dependent on the frequency modulation at the input.

The interferometers have two outputs, one is sent to a photodetector which converts the optical signal to an electrical signal. The other output has a 180 degree phase shifted interference pattern. Either output can be used for the analysis. To characterize the free spectral linewidth, two interferometers were used, one with a wavelength difference of 2.5 picometers, and one with a wavelength difference of 1.0 picometers.

The interferometers caused large enough amplitude fluctuations to make the predicted sinusoidal signal indistinguishable. Therefore, rather than linearly sweeping the frequency of the laser, the front and back mirror (FM and BM) currents were set to a known value and the phase mirror voltage was increased by $0.1 \mathrm{mV}$. The arbitrary waveform generators were set to DC bias the FM and BM with $4 \mathrm{~V}$. With this bias voltage, the FM current is $18.2 \mathrm{~mA}$ and the $\mathrm{BM}$ current is $24.4 \mathrm{~mA}$.

By adjusting the phase mirror current, the amplitude fluctuations were measured within the midrange of the interferometers, which can then be scaled back to a frequency fluctuation. Ideally, regardless of the interferometer free spectral range, the amplitude response should result in a constant level, which implies a constant frequency impulse. The derivation for the scale factor is provided in equations (3.10-3.12).

$$
\begin{gathered}
T(f)=\text { Vpeak } \sin \left(\frac{2 \pi}{\Delta f_{\text {int }}} f\right) \\
\frac{\partial T}{\partial f}=\operatorname{Vpeak}\left(\frac{2 \pi}{\Delta f_{\text {int }}}\right) \cos \left(\frac{2 \pi}{\Delta f_{\text {int }}} f\right)
\end{gathered}
$$




$$
\left.\frac{\partial T}{\partial f}\right|_{\max }=\operatorname{Vpeak}\left(\frac{2 \pi}{\Delta f_{\text {int }}}\right)
$$

The maximum transmission slope occurs at the midrange of the amplitude fluctuations, which allows the voltage fluctuations to be calculated from the following midrange plots in conjunction with the following formula:

$$
\text { line width } \stackrel{\text { def }}{=} \Delta f=\frac{\Delta V}{\left(\text { Vpeak } \frac{2 \pi}{\Delta f_{\text {int }}}\right)}
$$

Before these values can be computed, the interferometer difference frequencies $\left(\Delta f_{\text {int }}\right)$ have to be calculated from the following equations:

$$
\begin{gathered}
c=f \lambda \\
\Delta f_{\text {int }}=\Delta\left(\frac{c}{\lambda}\right) \\
\Delta f_{\text {int }}=\frac{c}{\lambda_{2}}-\frac{c}{\lambda_{1}} \\
\Delta f_{\text {int }}=\frac{c \lambda_{1}-c \lambda_{2}}{\lambda_{2} \lambda_{1}} \\
\Delta f_{\text {int }}=\Delta \lambda_{\text {int }}\left(\frac{-c}{\lambda_{2} \lambda_{1}}\right)
\end{gathered}
$$

The difference wavelength is the length of the interferometer delay and $\lambda_{1} \approx \lambda_{2}$ is approximately the center wavelength of the specific band. In this case for the L-Band laser, $\lambda \approx 1595 \mathrm{~nm}$.

The upper and midrange amplitude fluctuations for the interferometers are displayed in figures (3.16) through (3.19), where figure (3.15) depicts the corresponding measurement diagram. The upper range is used for the peak voltage in the previous equations and the midrange data is used for the voltage difference at the maximum slope. 


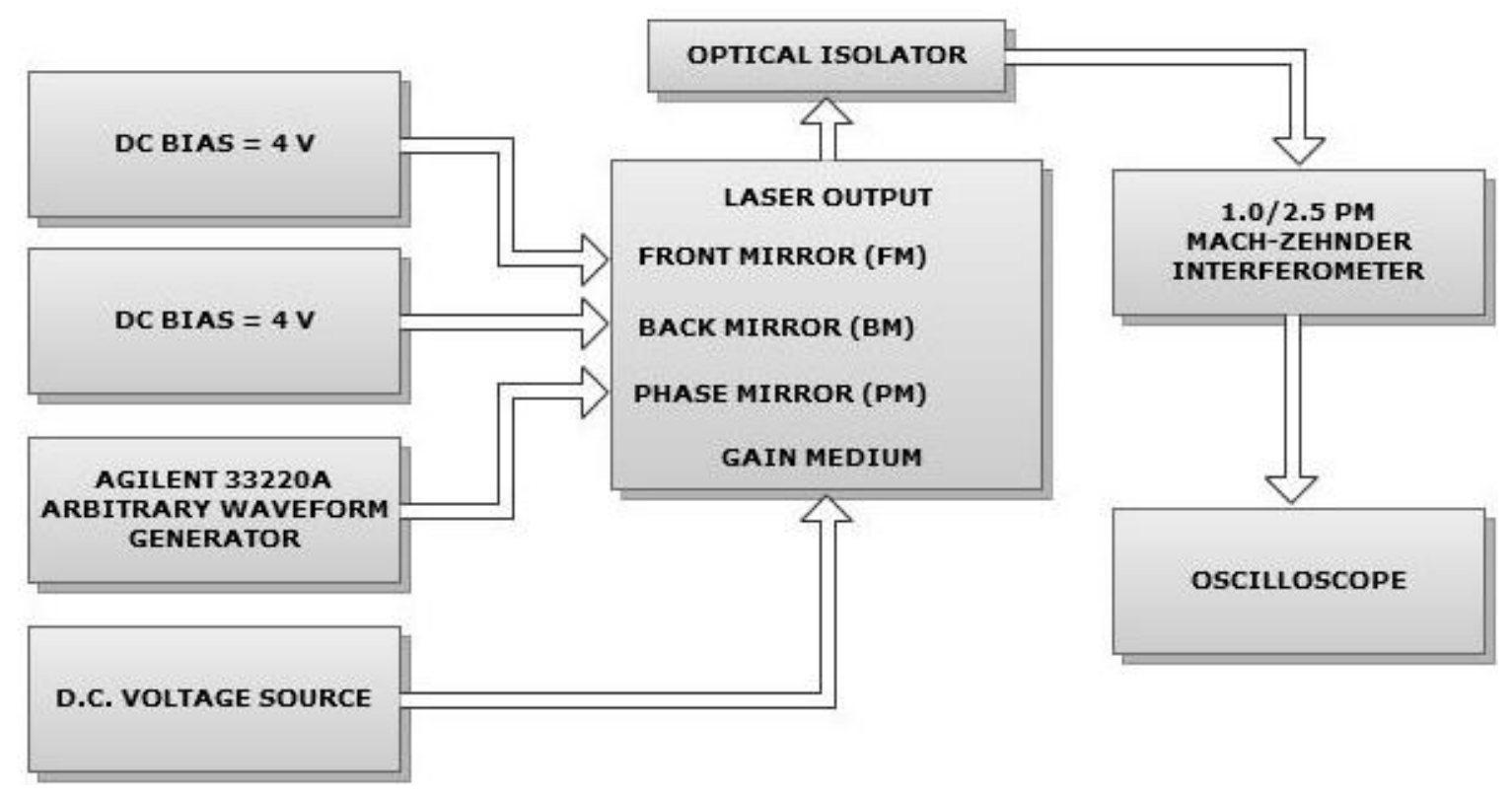

Figure 3.15: Frequency discriminator measurement diagram

Table 3.1: Difference wavelength and frequency data for two Mach-Zehnder Interferometers

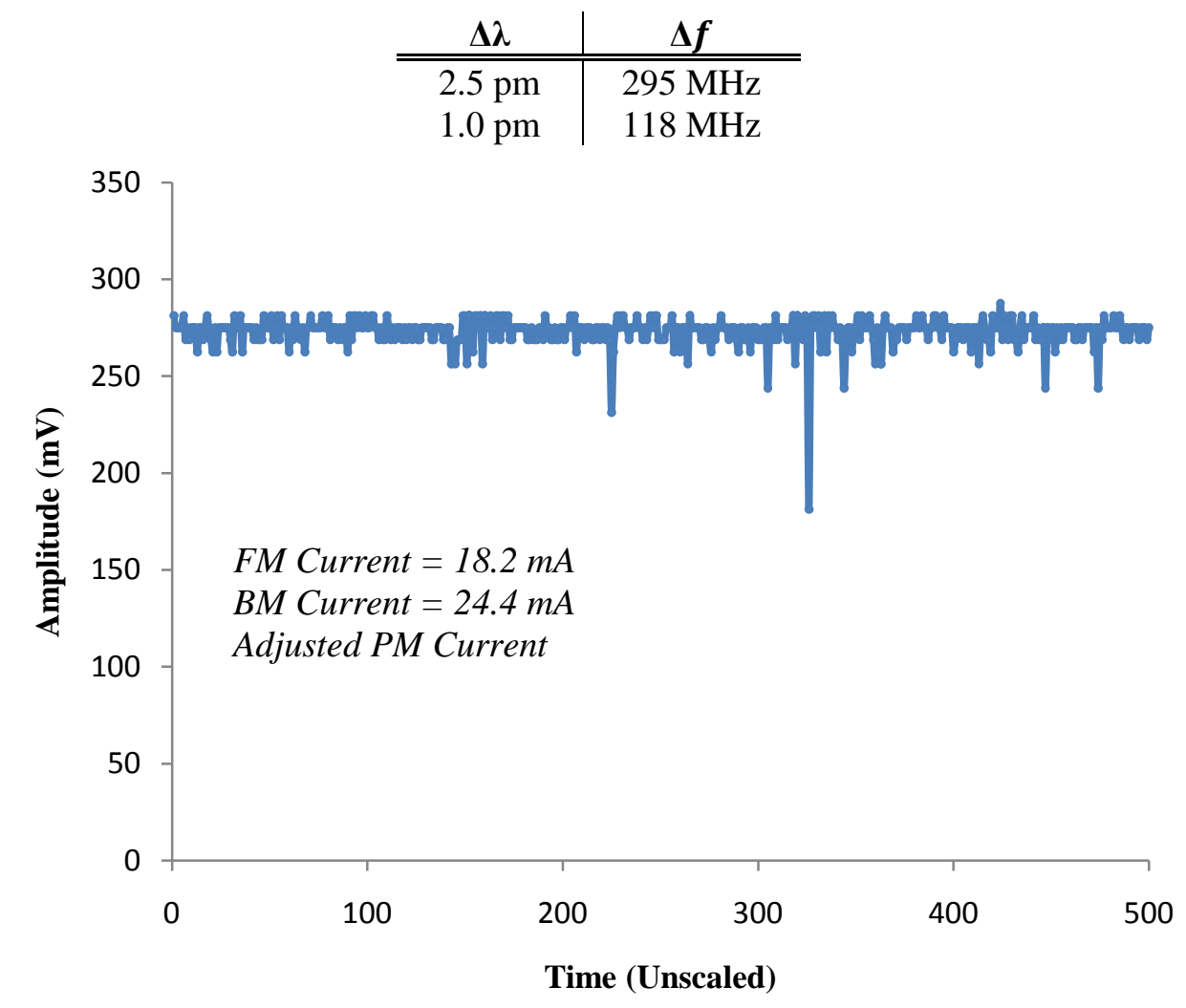

Figure 3.16: 2.5 pm Mach-Zehnder Interferometer upper range amplitude values, Vpeak $\approx 275 \mathrm{mV}$ 


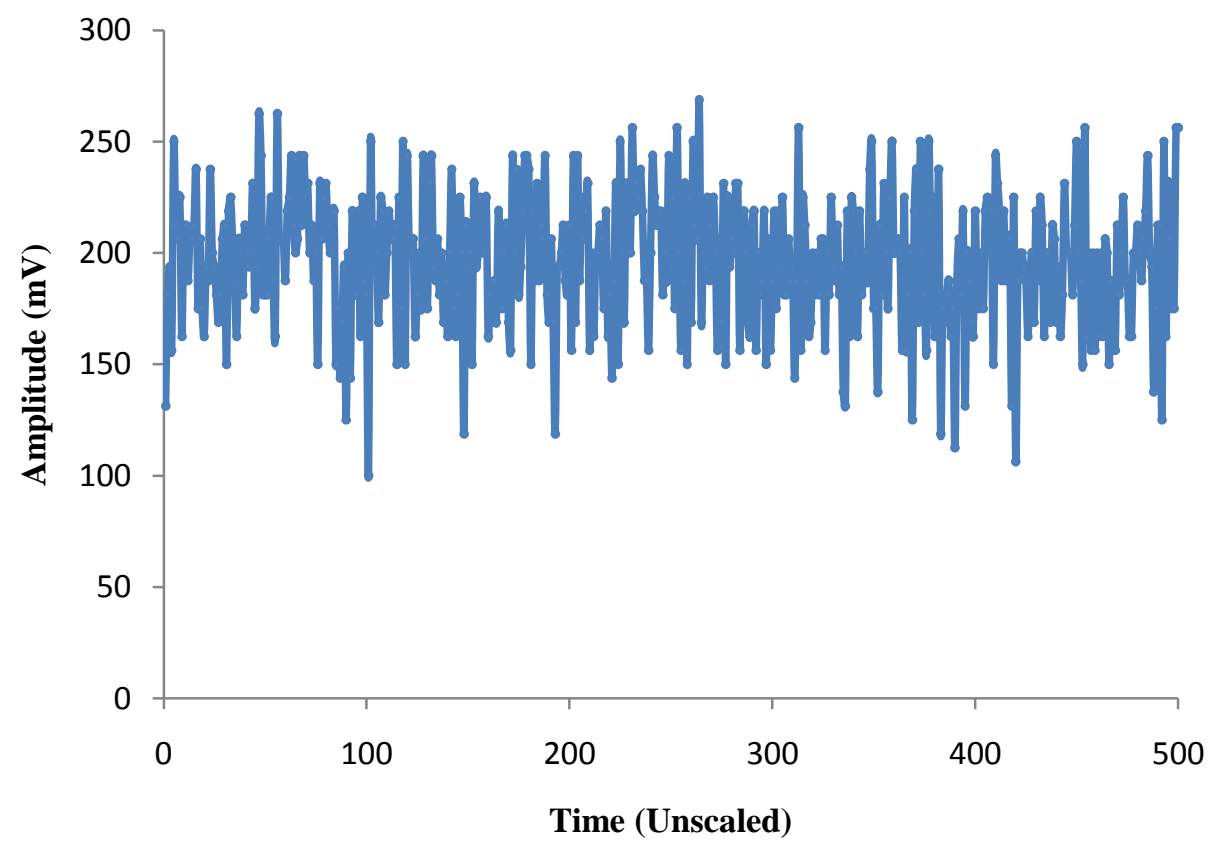

Figure 3.17: 2.5 pm Mach-Zehnder Interferometer midrange amplitude values, $\Delta \mathrm{V} \approx 100 \mathrm{mV}$

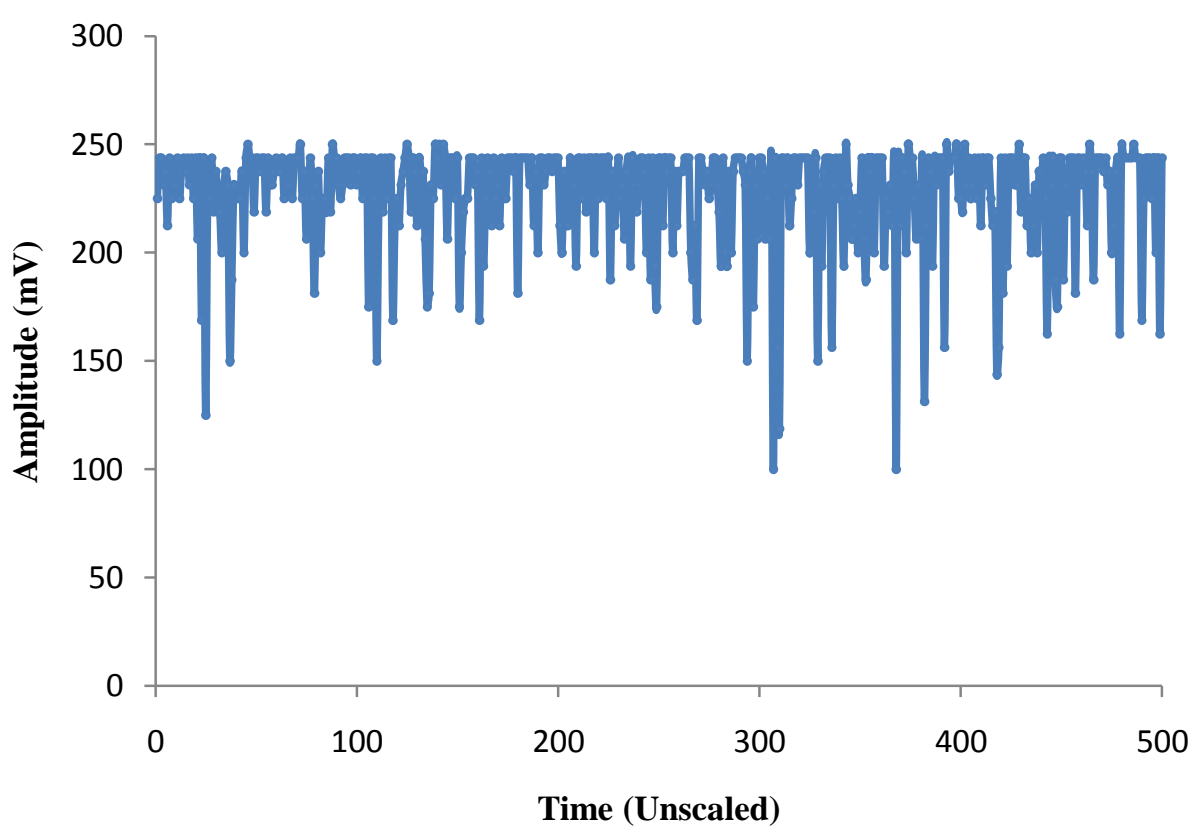

Figure 3.18: 1.0 pm Mach-Zehnder Interferometer upper range amplitude values, Vpeak $\approx 240 \mathrm{mV}$ 


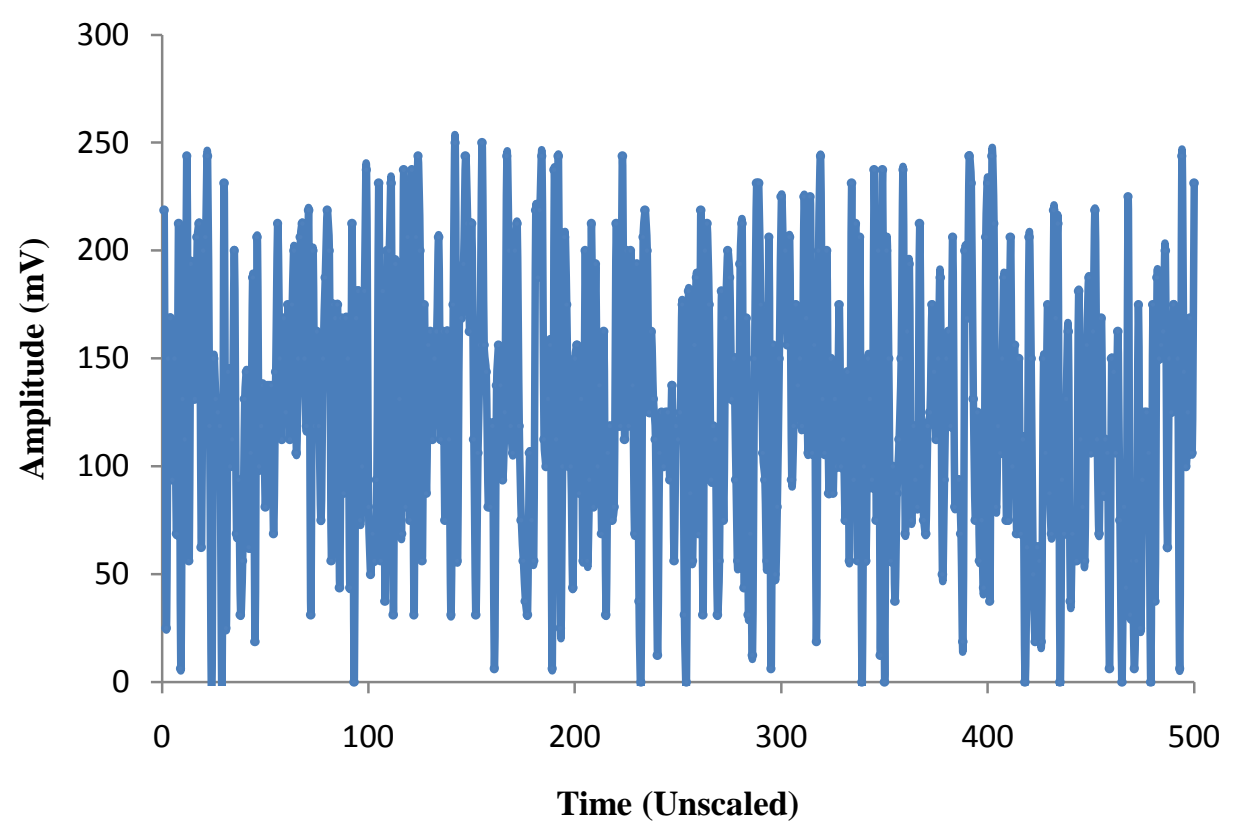

Figure 3.19: $1.0 \mathrm{pm}$ Mach-Zehnder Interferometer midrange amplitude values, $\Delta \mathrm{V} \approx 210 \mathrm{mV}$

2.5 pm Line Width Calculation:

$$
\begin{gathered}
\text { line width } \equiv \Delta f=\frac{100 \mathrm{mV}}{\left(275 \mathrm{mV} \frac{2 \pi}{295 \mathrm{MHz}}\right)} \\
\text { line width } \equiv \Delta f \approx 17.1 \mathrm{MHz}
\end{gathered}
$$

1.0 pm Line Width Calculation:

$$
\begin{gathered}
\text { line width } \equiv \Delta f=\frac{240 \mathrm{mV}}{\left(210 \mathrm{mV} \frac{2 \pi}{118 \mathrm{MHz}}\right)} \\
\text { line width } \equiv \Delta f \approx 21.5 \mathrm{MHz}
\end{gathered}
$$

By averaging the results from the interferometers, the linewidth is tested to be approximately $20 \mathrm{MHz}$. SG-DBR lasers typically have a narrow line width; therefore, this value is a reasonable characterization. This value also imposes an interpolation restraint important to a proper calibration which is evident in chapter 5 where a $100 \mathrm{MHz}$ frequency spacing interpolation is implemented. 


\subsection{2 - Tuning Pad Side Mode Suppression Ratio (SMSR)}

The side mode suppression ratio of a laser with a particular center wavelength is defined as the amplitude difference in $\mathrm{dB}$ between the main spectral component and the largest side mode [19]. The power in the sidebands occurs from a second order interference pattern produced from a larger transmission through each mirror. As the light travels through the gain medium, the electromagnetic wave reflects off the mirrors and also interferes with the previously reflected wave, which creates an exponentially higher loss spectrum above and below the center wavelength.

With a SG-DBR laser, it is important to tune the front and back mirror currents to operate in a mode with equal upper and lower side bands. A normally weak mode becomes stronger as the currents are increased, which moves the point of interest away from the optimum SMSR. At a mode intersection, the SMSR is at its worst and any further tuning will cause the laser to change modes; the weak mode takes over and becomes the new main mode [20].

To characterize the appropriate point on a constant wavelength tuning pad, the upper and lower side mode suppression is examined as a function of linear FM and BM current. Theoretically, the equal side mode suppression occurs at the midpoint of currents, which lies directly in the center of the pads.

To calculate the SMSR of the L-Band laser, path number 5 from figure (3.21) was chosen. The front and back mirror currents were linearly increased along the line on the third tuning pad from the right. This was automated with a LabVIEW program that changed the currents based on a set number of samples and recorded each trace from the optical spectrum analyzer. 
The upper and lower sideband fluctuated in amplitude as initially expected. As current was linearly increased along the tuning pad as seen in figure (3.21), the lower sideband increased, until the current reached the next wavelength mode. At this point, the spectrum frequently jittered back and forth between modes. Likewise, as the current decreased, the upper sideband increased.

To achieve a complete tuning pad characterization, the sideband magnitudes are plotted against a straight line sweep of FM and BM current. The side mode suppression ratio is the difference between the main spectral component strength $(10 \mathrm{dBm})$ and the magnitude data on the following page.

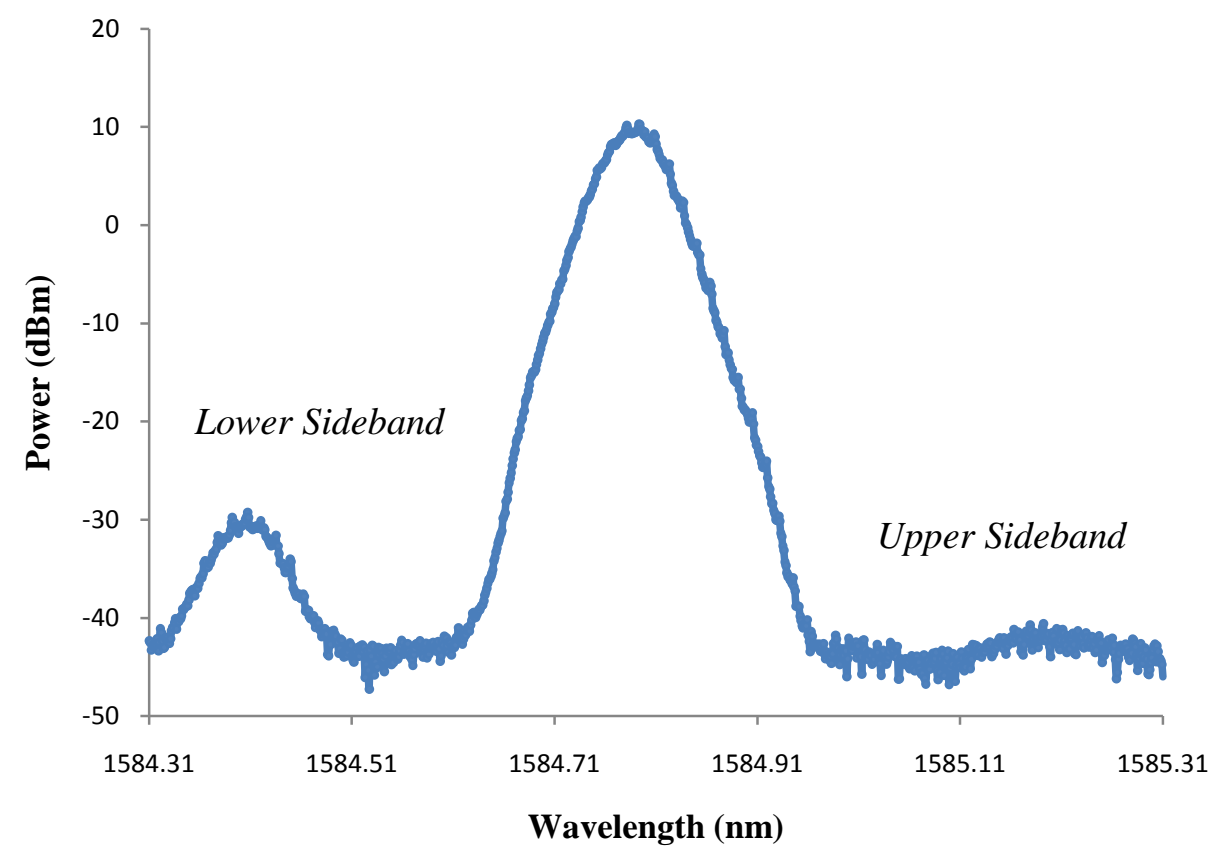

Figure 3.20: Example of a lower sideband with small side mode suppression ratio. Both upper and lower peaks should ideally be equal. 


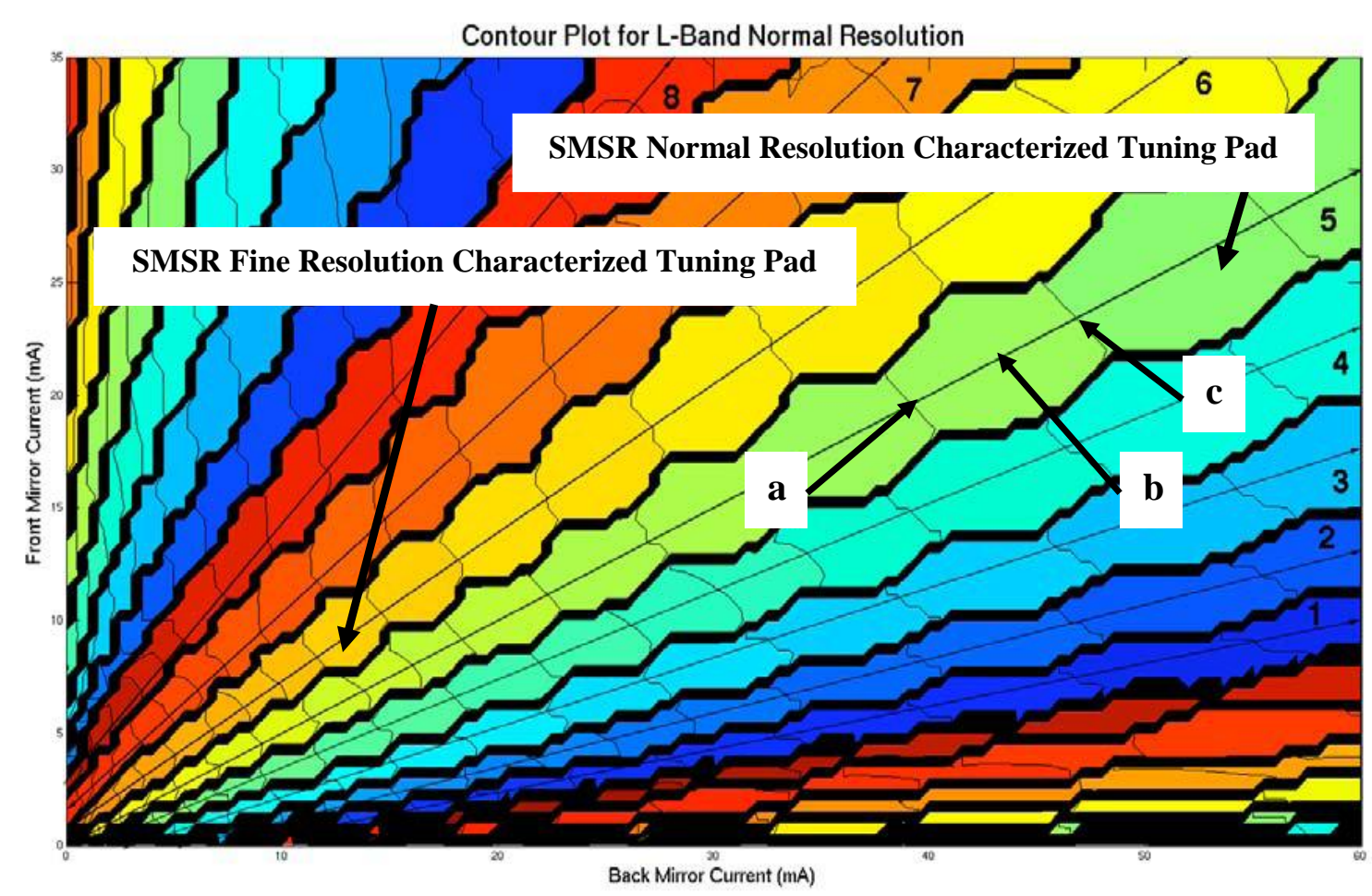

Figure 3.21: Contour plot for L-band laser showing normal and fine resolution characterization up to the maximum current in the mirrors (the various colors represent specific wavelength modes) [15]

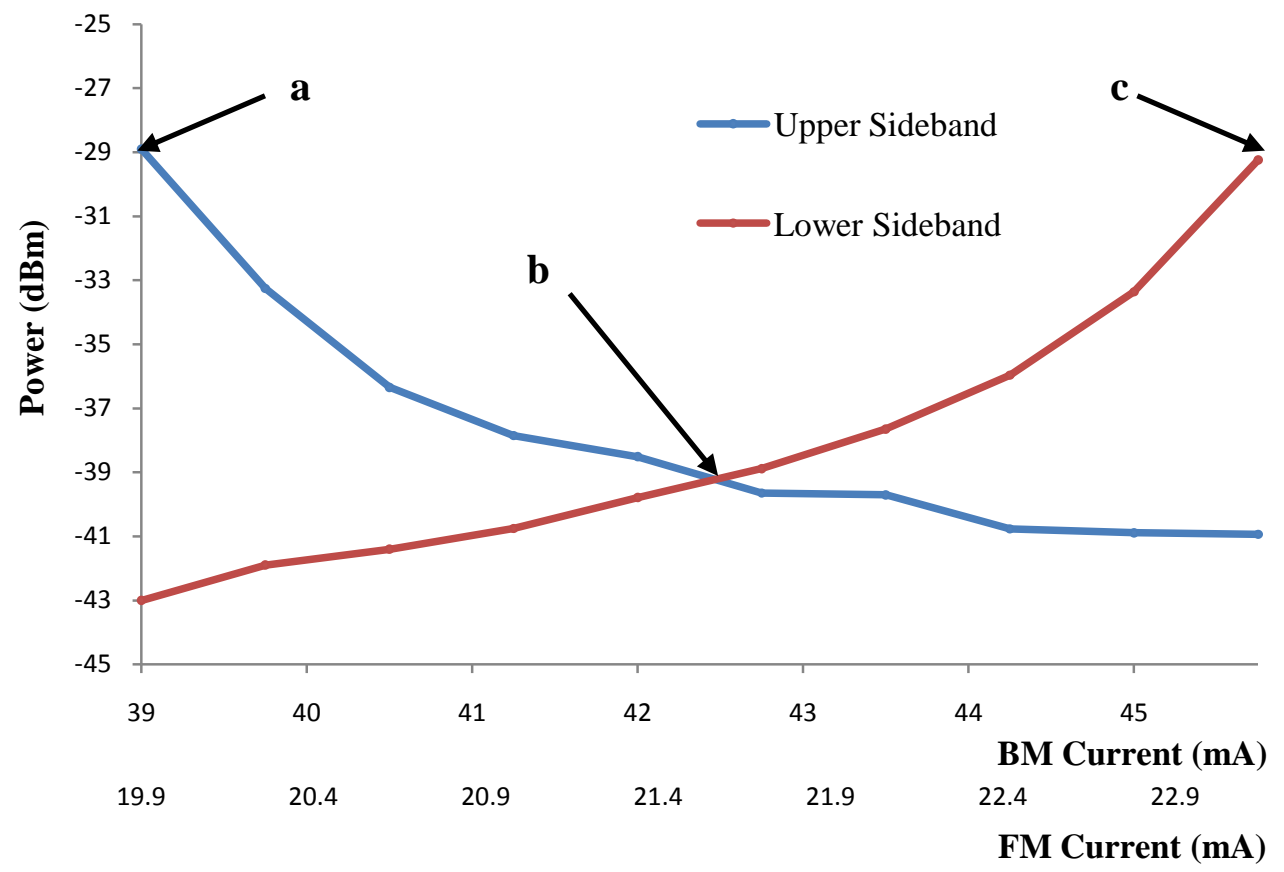

Figure 3.22: Graph of sideband magnitude as a function of front and back mirror current. This data is taken along path 5 for the $L$-Band laser where points $a, b$, and $c$ represent the corresponding locations. 
The deviation can be modeled by a straight line. The sidebands respond inversely to each other; therefore, it isn't necessary to solve for each band sensitivity. Equation (3.23) represents the SMSR as a function of FM current for figure (3.22).

$$
\begin{gathered}
\left|\frac{45.96 \mathrm{~dB}-51.40 \mathrm{~dB}}{22.42 m A-20.62 m A}\right|=3.02 \mathrm{~dB} / \mathrm{mA} \\
\left(\frac{1.8 m A}{24.0 m A-18.4 m A}\right) * 100 \%=32.1 \% \\
\left(\frac{3.02 \mathrm{~dB}}{m A}\right) *\left(\frac{1.8 m A}{32.1 \%}\right)=0.169 \mathrm{~dB} / \%
\end{gathered}
$$

If the SMSR restraint is set to $45 \mathrm{~dB}$, it allows a deviation from the difference of the SMSR at equal suppression away from the center by a percentage range. SMSR at equal suppression is equal to $50 \mathrm{~dB}$. Therefore, this allows a $5 \mathrm{~dB}$ deviation. In terms of a percentage away from the center based on current, this results in a $30 \%$ deviation along the straight line path for figure (3.21).

Table 3.2: Data representing the sideband deviation as a function of current

\begin{tabular}{c|c|c|c|c} 
FM (mA) & BM (mA) & LSB (dBm) & USB $(\mathbf{d B m})$ & $\mathbf{C W}(\mathbf{d B m})$ \\
\hline \hline 19.90 & 39.00 & -43.00 & -28.90 & 10.69 \\
20.26 & 39.75 & -41.89 & -33.26 & 10.37 \\
20.62 & 40.50 & -41.40 & -36.34 & 10.35 \\
20.98 & 41.25 & -40.75 & -37.85 & 10.28 \\
\hline 21.34 & 42.00 & -39.79 & -38.51 & 10.51 \\
21.70 & 42.75 & -38.88 & -39.65 & 10.57 \\
\hline 22.06 & 43.50 & -37.65 & -39.70 & 10.24 \\
22.42 & 44.25 & -35.96 & -40.77 & 10.29 \\
22.78 & 45.00 & -33.36 & -40.89 & 10.28 \\
23.14 & 45.75 & -29.24 & -40.94 & 10.30
\end{tabular}


This value is in accordance with a $100 \%$ deviation relating to the edge of a tuning pad. For example, equation (3.26) relates the full deviation in terms of SMSR (dB):

$$
100 \% *\left(\frac{0.169 d B}{1.0 \%}\right)=16.9 d B
$$

As expected, the $16.9 \mathrm{~dB}$ deviation relates to the difference between the equal SMSR and the case where one of the sidebands is dominate.

$$
-23.0 \mathrm{dBm}+39.34 \mathrm{dBm}=16.34 \mathrm{~dB}
$$

The SMSR deviates by approximately $0.17 \mathrm{~dB} / 1.0 \%$ from the center. Equal SMSR occurs at the middle of the tuning pad and is approximately equal to $50 \mathrm{~dB}$. 


\subsection{3 - Fine Resolution SMSR}

When compared to the normal resolution current SMSR characterization, the fine resolution (lower bias current modes) data has the same characteristics. The graph below shows the SMSR of the upper and lower sidebands versus a linear progression through a smaller tuning pad on path 6 for the L-Band laser. It's important to note that this graph provides the actual side mode suppression ratio as opposed to the normal characterization where the sideband magnitudes are included.

In the following chapter, the SMSR characterization will also be compared to the gain medium voltage response to further verify an optimal tuning path for automatic calibration.

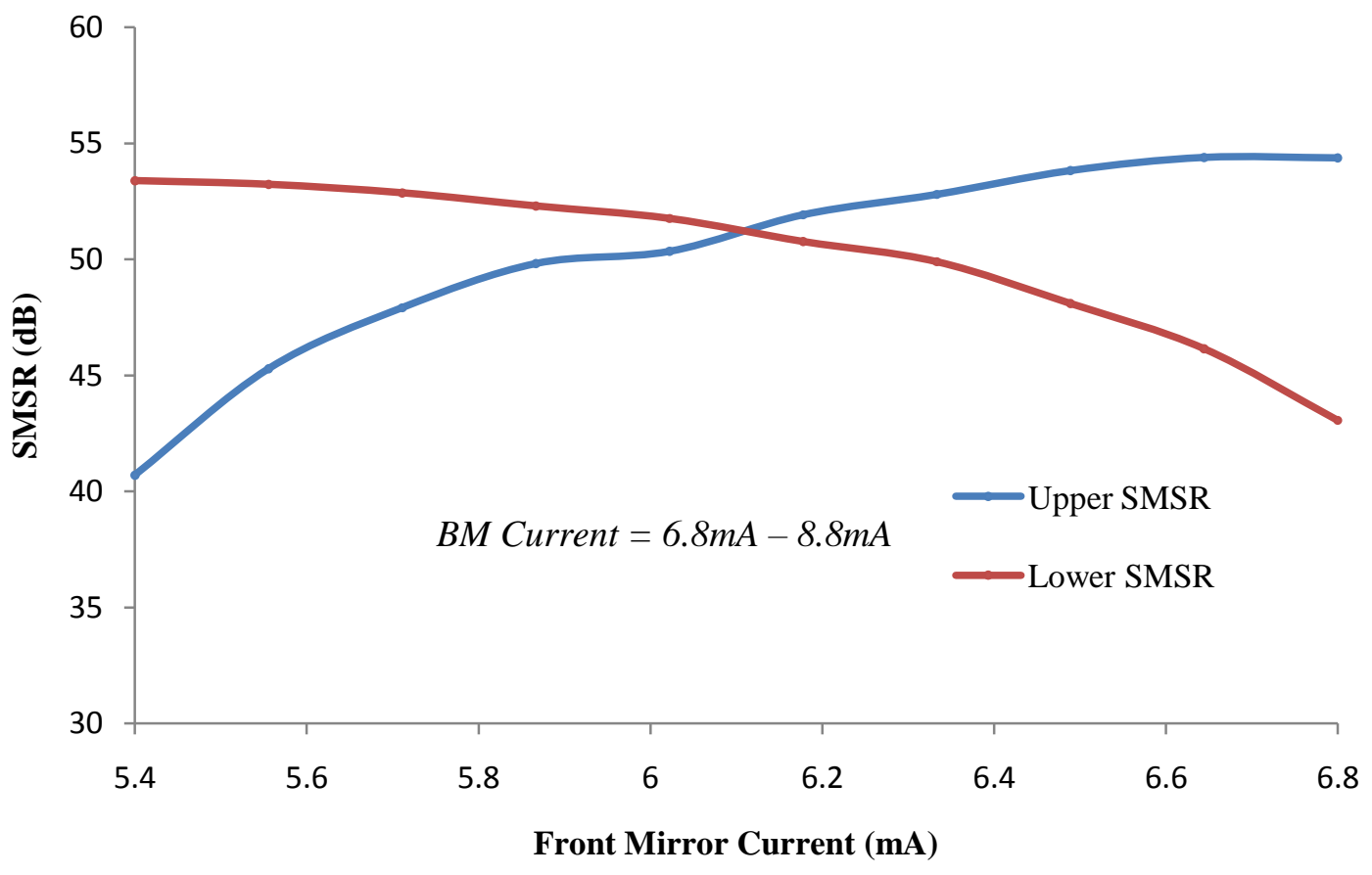

Figure 3.23: Graph of sideband magnitude as a function of front and back mirror current for fine resolution. This data is taken along path 6 for the L-Band laser as seen in figure (3.21). 


\section{CHAPTER 4 - Gain Medium Voltage Implications}

Now that the operation of the SG-DBR laser has been explained, the gain medium voltage response is analyzed. The results of this analysis provide the needed platform to allow for an automated calibration procedure that can be performed in a time efficient manner. Throughout this chapter, a qualitative background will support the subsequent voltage characterizations and a determination of threshold levels will be provided.

\section{1 - Instantaneous Voltage Change at Wavelength Mode Transitions}

Instantaneous voltage changes occur within the gain medium of the laser due to wavelength mode hops. As previously discussed, depending on the characterization dimensionality (single mirror, or simultaneous front and back mirror) the laser will output discrete increments of constant wavelengths. These constant wavelength modes versus front and back mirror currents are provided as a contour plot in figure (4.1). The different colors represent corresponding wavelengths. Each outlined pad corresponds to a constant wavelength. Tuning from path to path results in $5 \mathrm{~nm}$ mode hops (onedimensional characterization), whereas tuning along the numbered paths results in $0.3 \mathrm{~nm}$ mode hops (two-dimensional characterization). These contour plots provide the needed framework to visually validate the mirror tuning background sections in chapter 3 and provide a comparison to the voltage responses within this chapter.

Each pad corresponds to a longitudinal cavity mode of the laser. For example, as the phase current is tuned along the continuous $0.2 \mathrm{~nm}$ path, the internal cavity length changes. The laser is continuously attempting to maintain an integer number of half wavelengths of light within the cavity, such that equation (4.1), where $\mathrm{N}$ is an integer, $\lambda$ is the wavelength, and $\mathrm{L}$ is the cavity length, is always satisfied. 


$$
N\left(\frac{\lambda}{2}\right)=N(L)
$$

While this condition is satisfied, the threshold current remains low, but as mode competition (wavelength mode hops) occur, the laser becomes unstable. This instability causes significant output power and gain medium voltage fluctuations which are related to the mirror loss. Once the mode hop occurs, the current increases transiently for a brief period of time before the adjacent mode begins to dominate, which changes the required gain. Since gain is proportional to carrier density in the active region and the gain medium voltage is dependent on carrier density, this transient increase in laser current is what leads to instantaneous voltage changes at mode transitions. This occurrence ultimately leads to voltage thresholds used for automating calibration. The experimental voltage responses will now be presented throughout the remainder of this chapter.

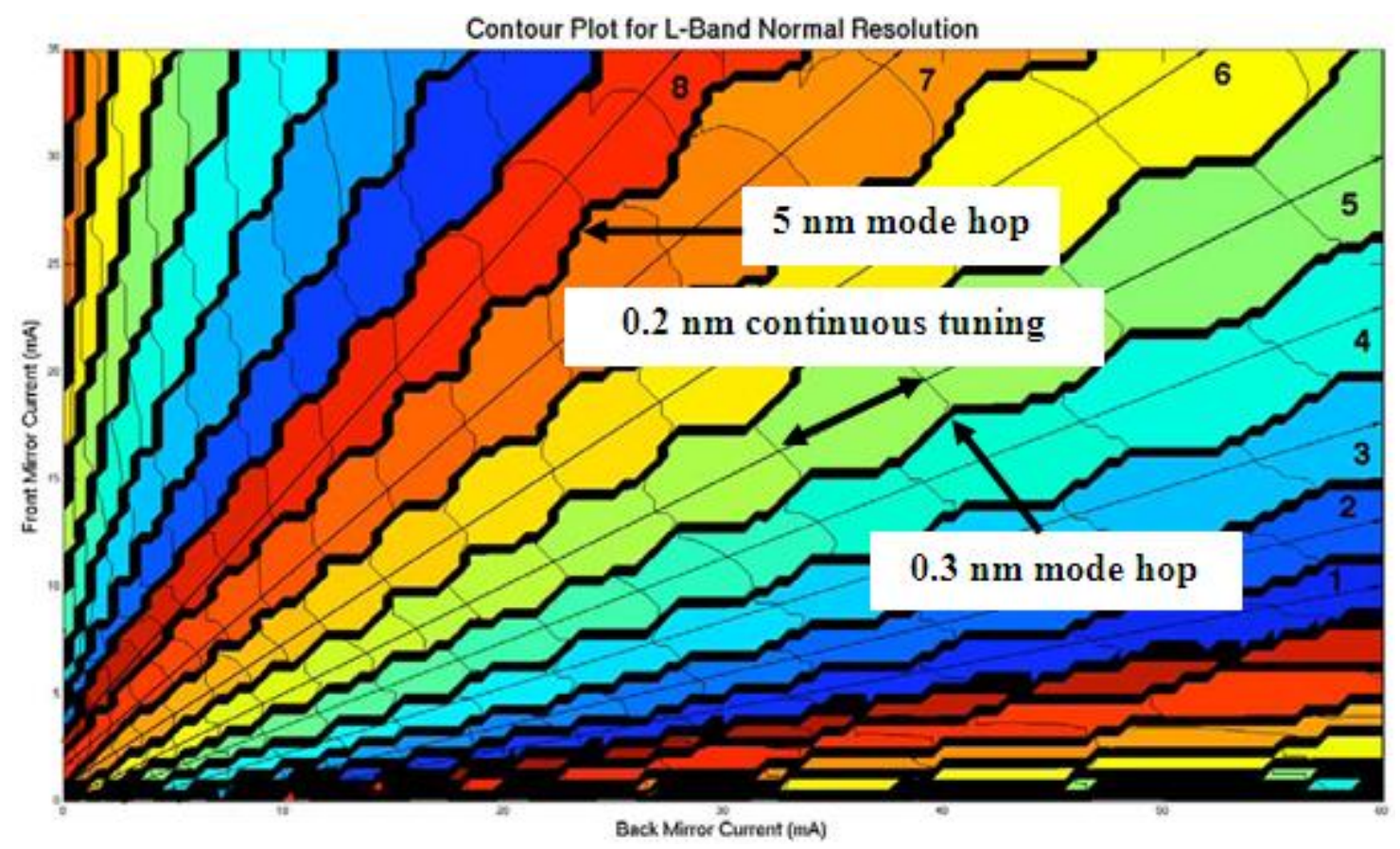

Figure 4.1: Contour plot for $L$-band laser showing normal resolution characterization up to the maximum current in the mirrors. Note the eight tuning paths covering low wavelengths to high wavelengths [15]. 


\section{2 - Two-Dimensional Characterization}

The two-dimensional voltage response is tested with the front and back mirror currents changing simultaneously. The first tested response is the linear progression over one wavelength pad. The same pad used in subsection (3.8.2) in path 5 is tested for SMSR comparison purposes which will be analyzed in subsection (4.2.1). The purpose of this test is to further understand the voltage relationship with the various mode changes. The results for a single two-dimensional pad change will be provided as well as voltage responses for path 1 and path 5 as seen in figure (3.21) within both C and L-Band lasers. Also, a hysteresis analysis will be included to gain a better understanding of the automated capabilities. Figure (4.2) demonstrates a single pad two-dimensional voltage response characteristic. Figures (4.3-4.12) show the two-dimensional voltage responses and their respective first and second order derivative characteristics.

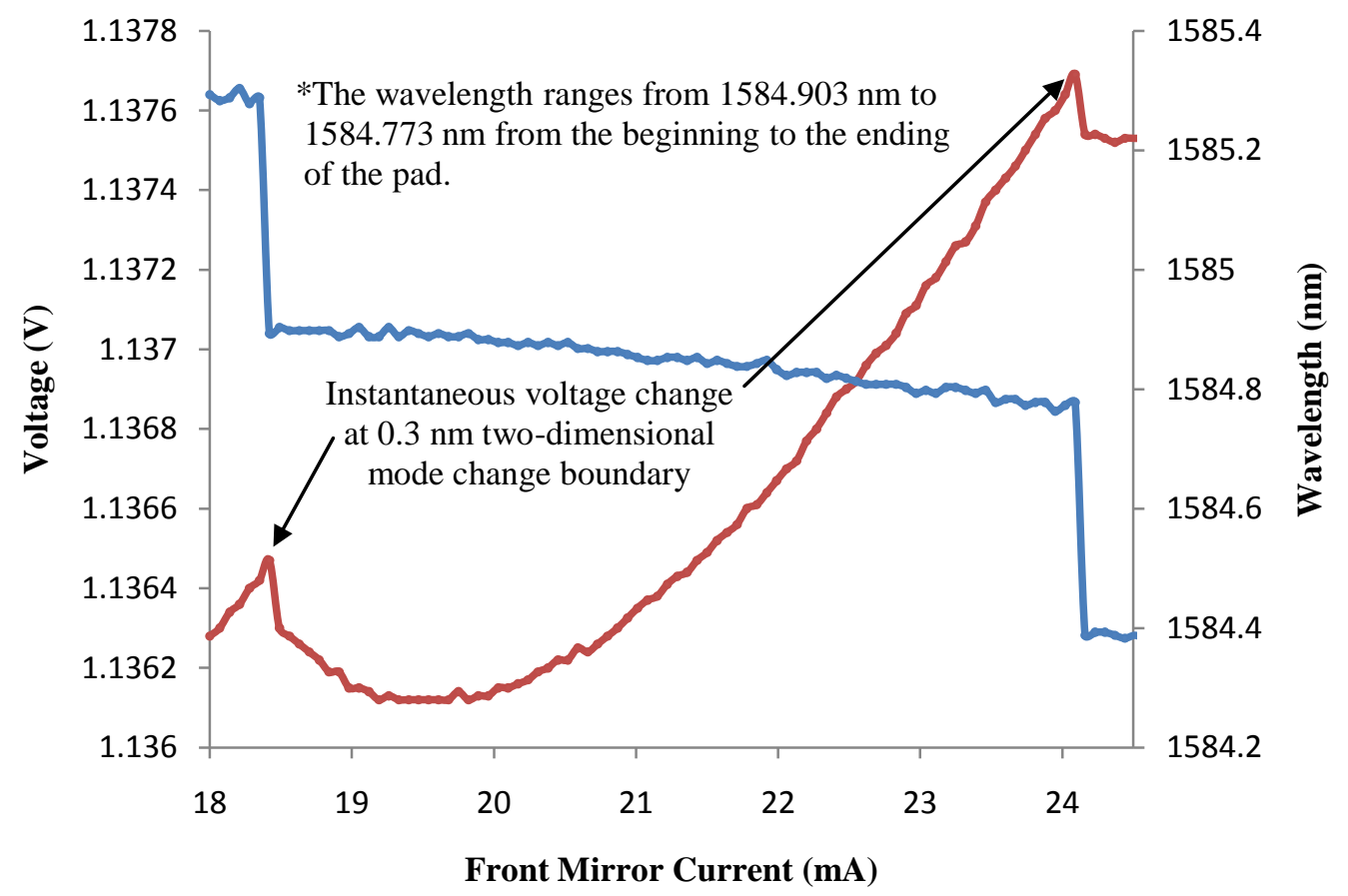

Figure 4.2: Voltage response and wavelength with a linear progression of increasing front and back mirror current within the corresponding pad in path 5 of the L-Band laser depicted in figure (3.21) 


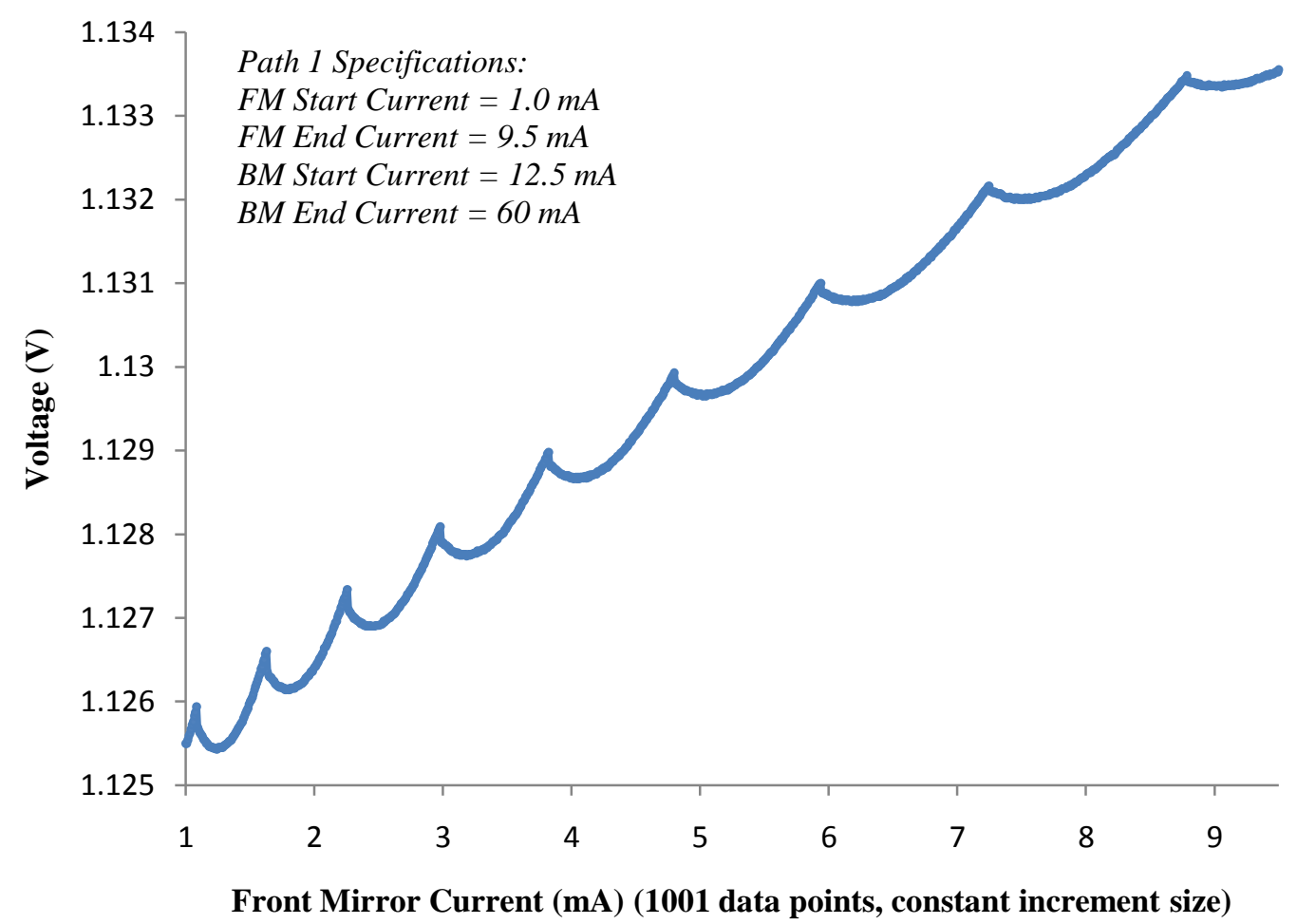

Figure 4.3: Voltage response for L-Band path 1 depicted in figure (4.18)

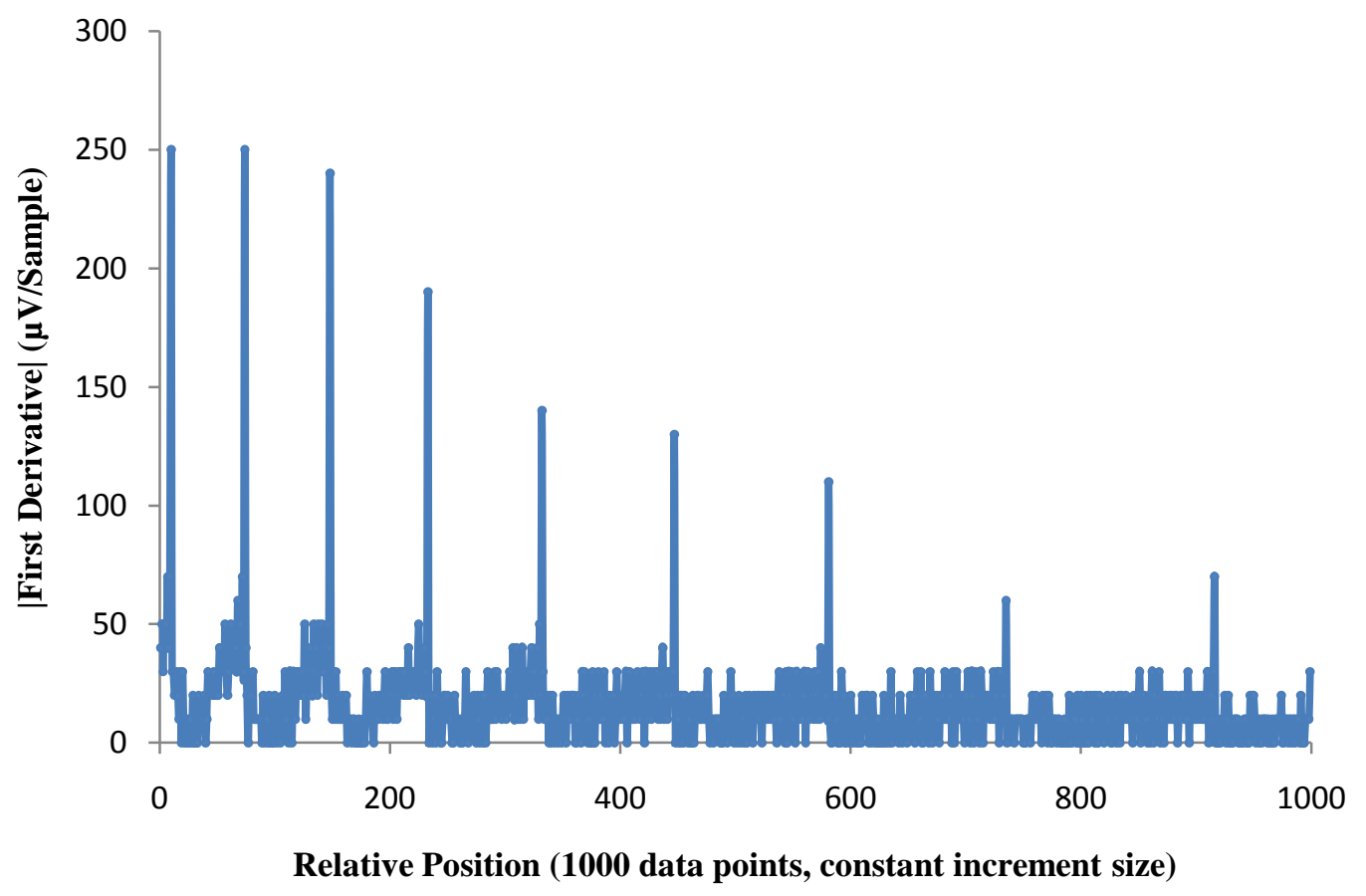

Figure 4.4: Absolute value of first derivative voltage response for $L$-Band path 1 


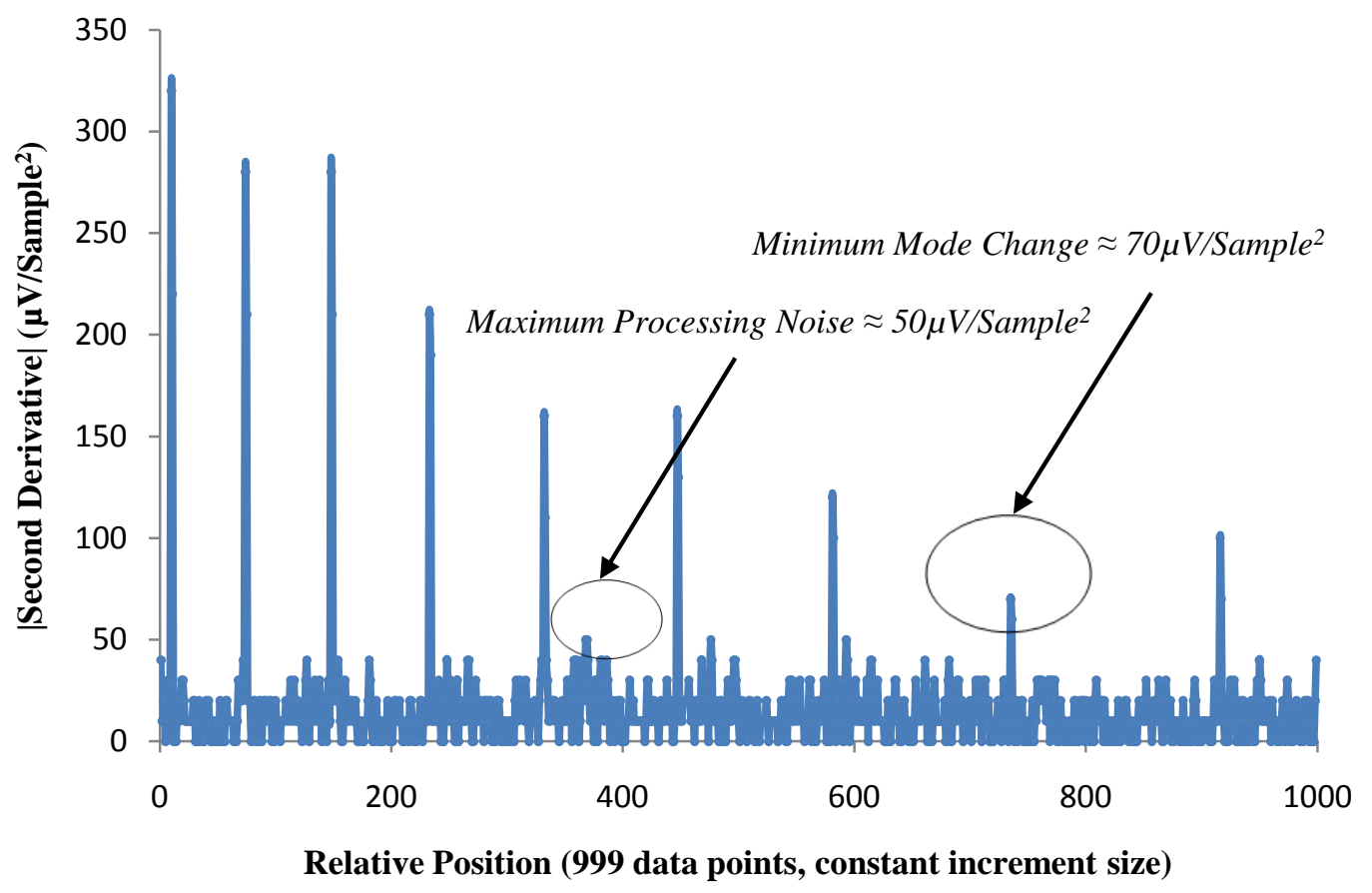

Figure 4.5: Absolute value of second derivative voltage response for L-Band path 1

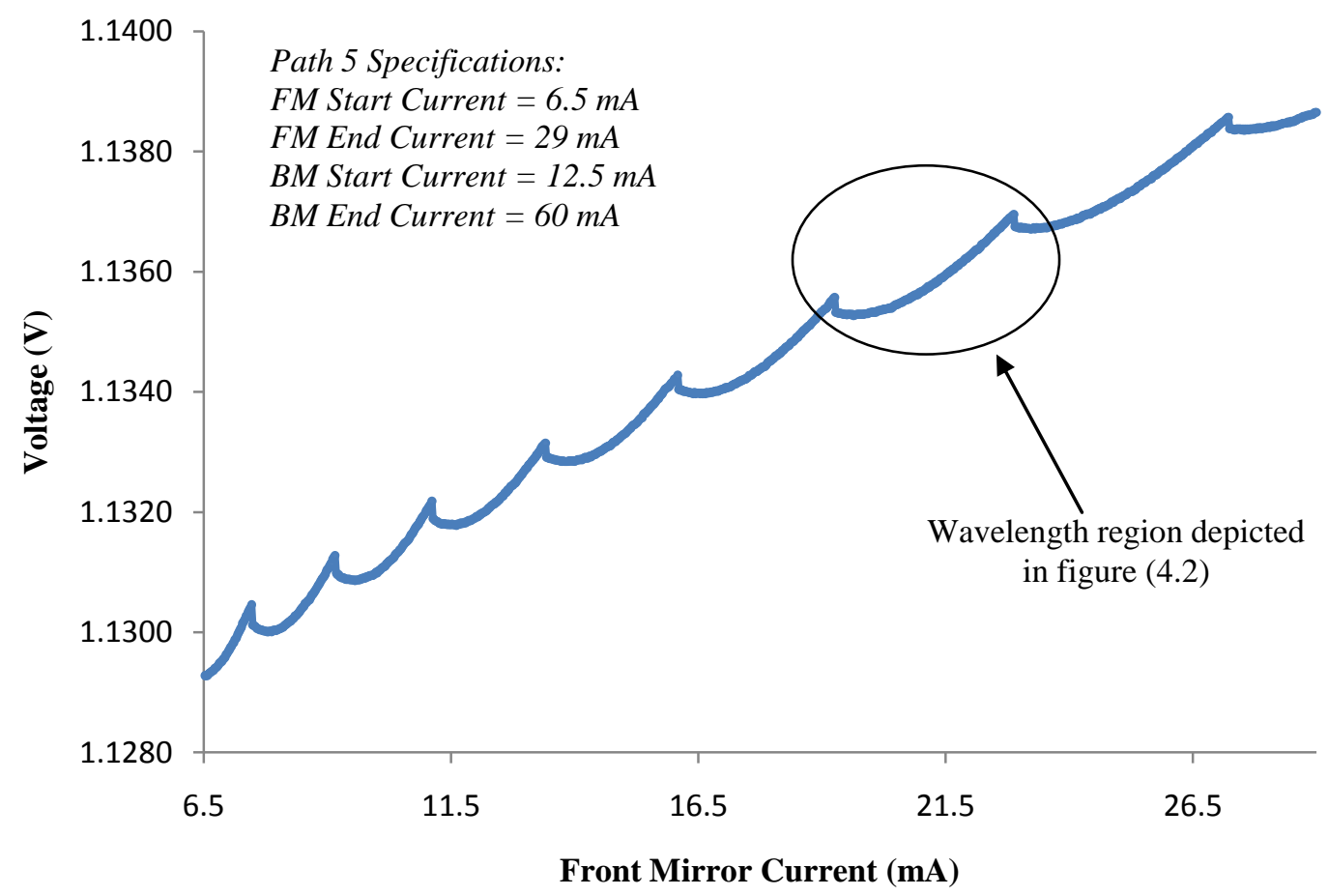

Figure 4.6: Voltage response for L-Band path 5 depicted in figure (4.18) 


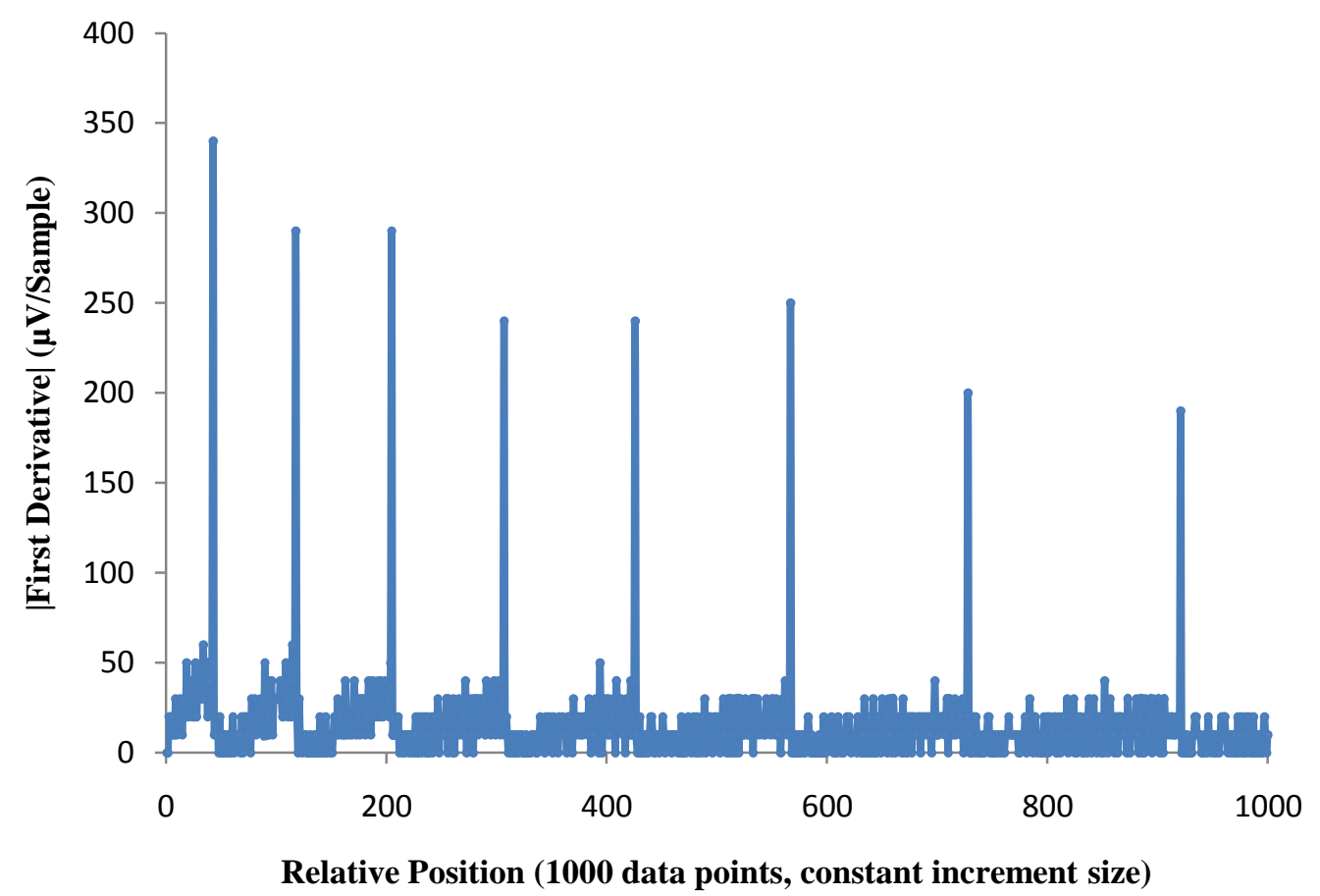

Figure 4.7: Absolute value of first derivative voltage response for L-Band path 5

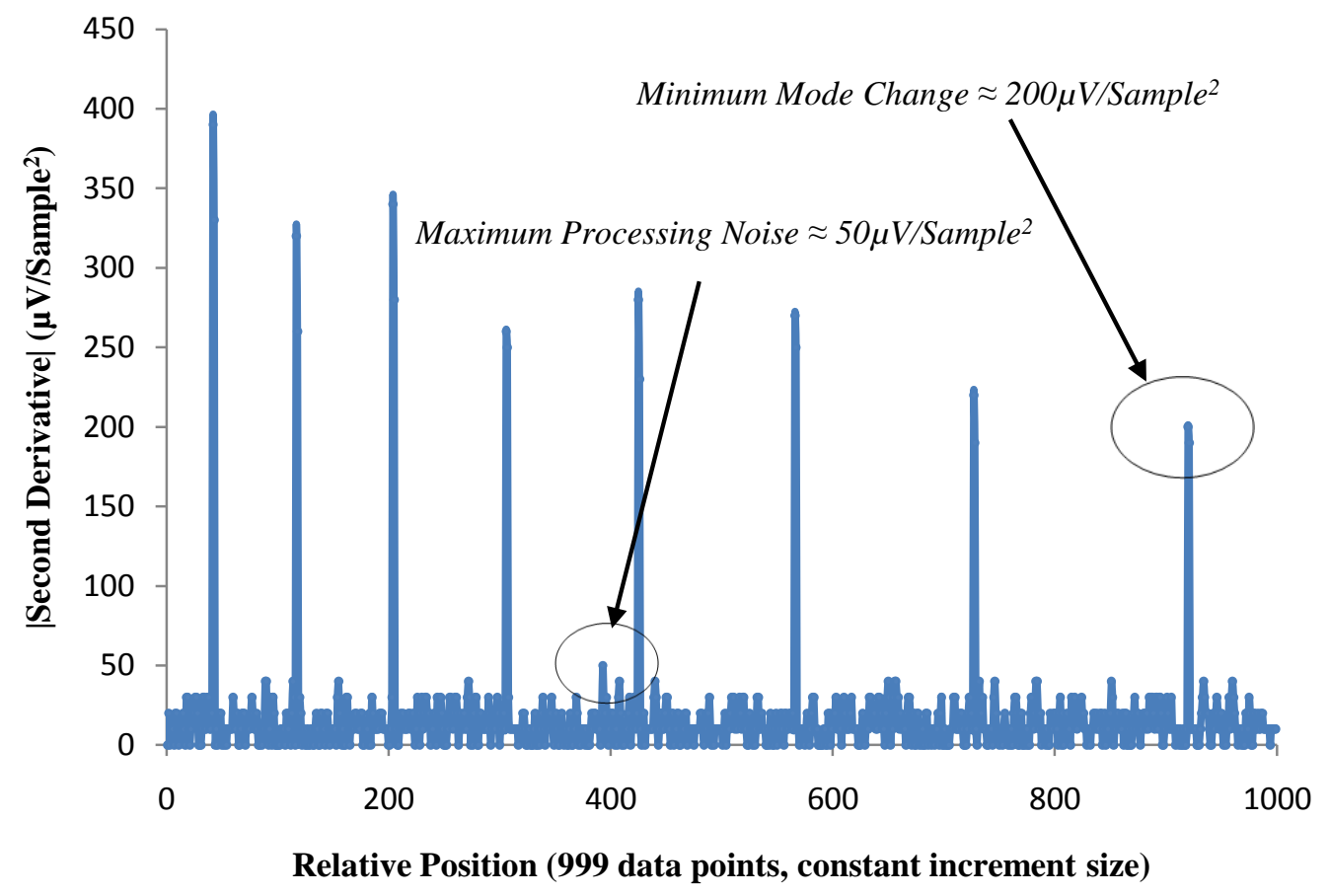

Figure 4.8: Absolute value of second derivative voltage response for L-Band path 5 


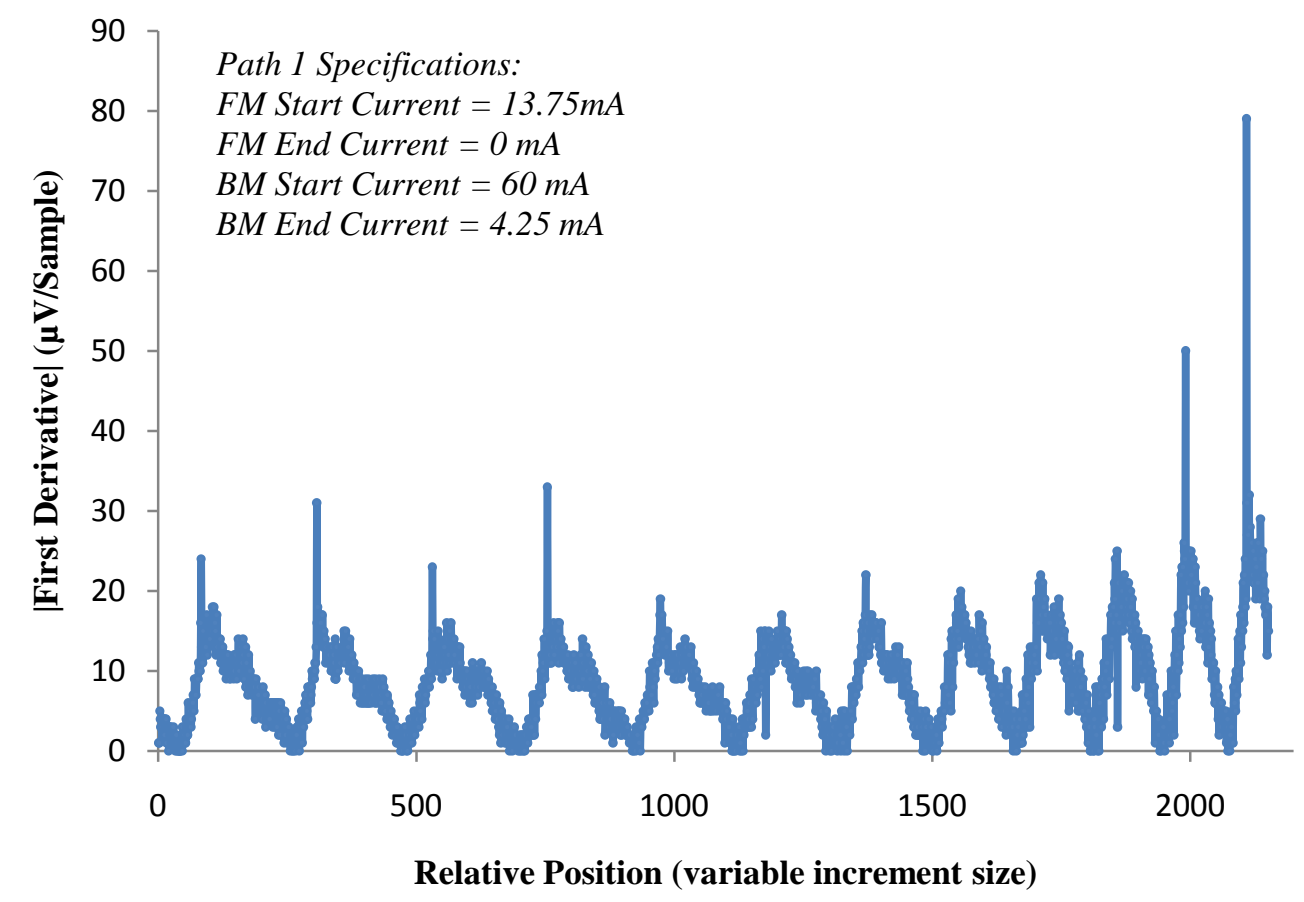

Figure 4.9: Absolute value of first derivative voltage response for $\mathrm{C}$-Band path 1 which corresponds to the same general location depicted in figure (4.18)

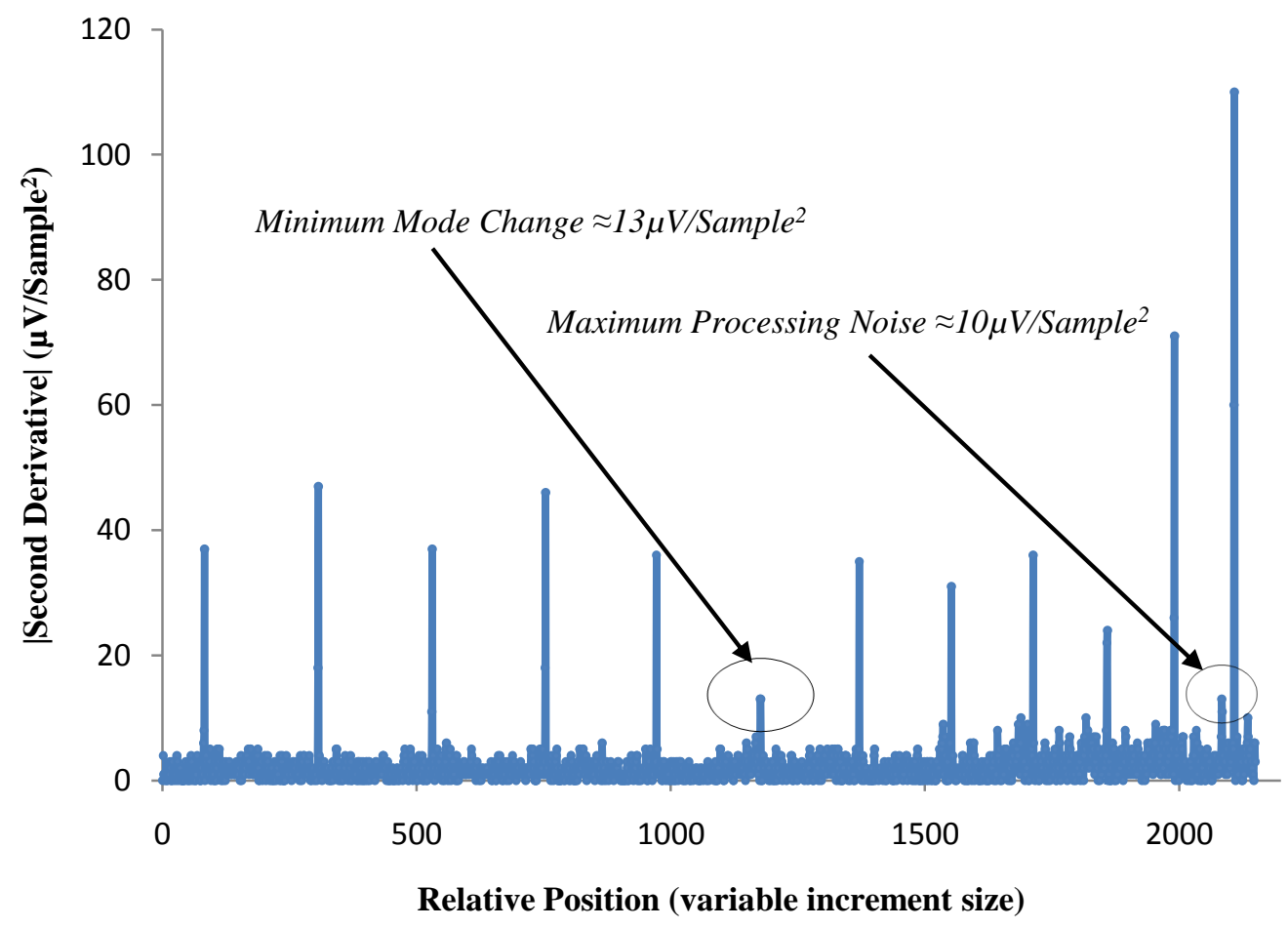

Figure 4.10: Absolute value of second derivative voltage response for C-Band path 1 


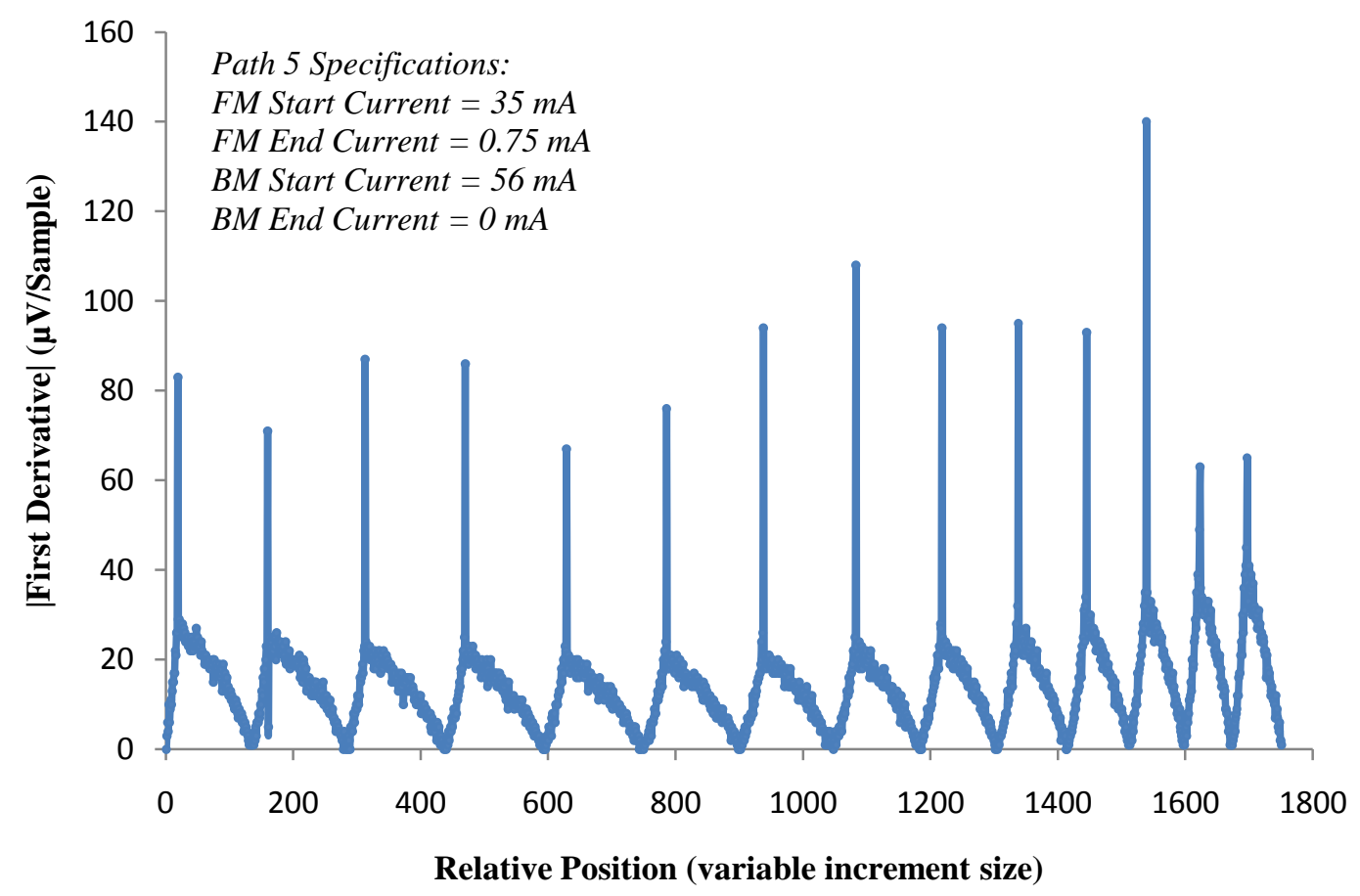

Figure 4.11: Absolute value of first derivative voltage response for C-Band path 5

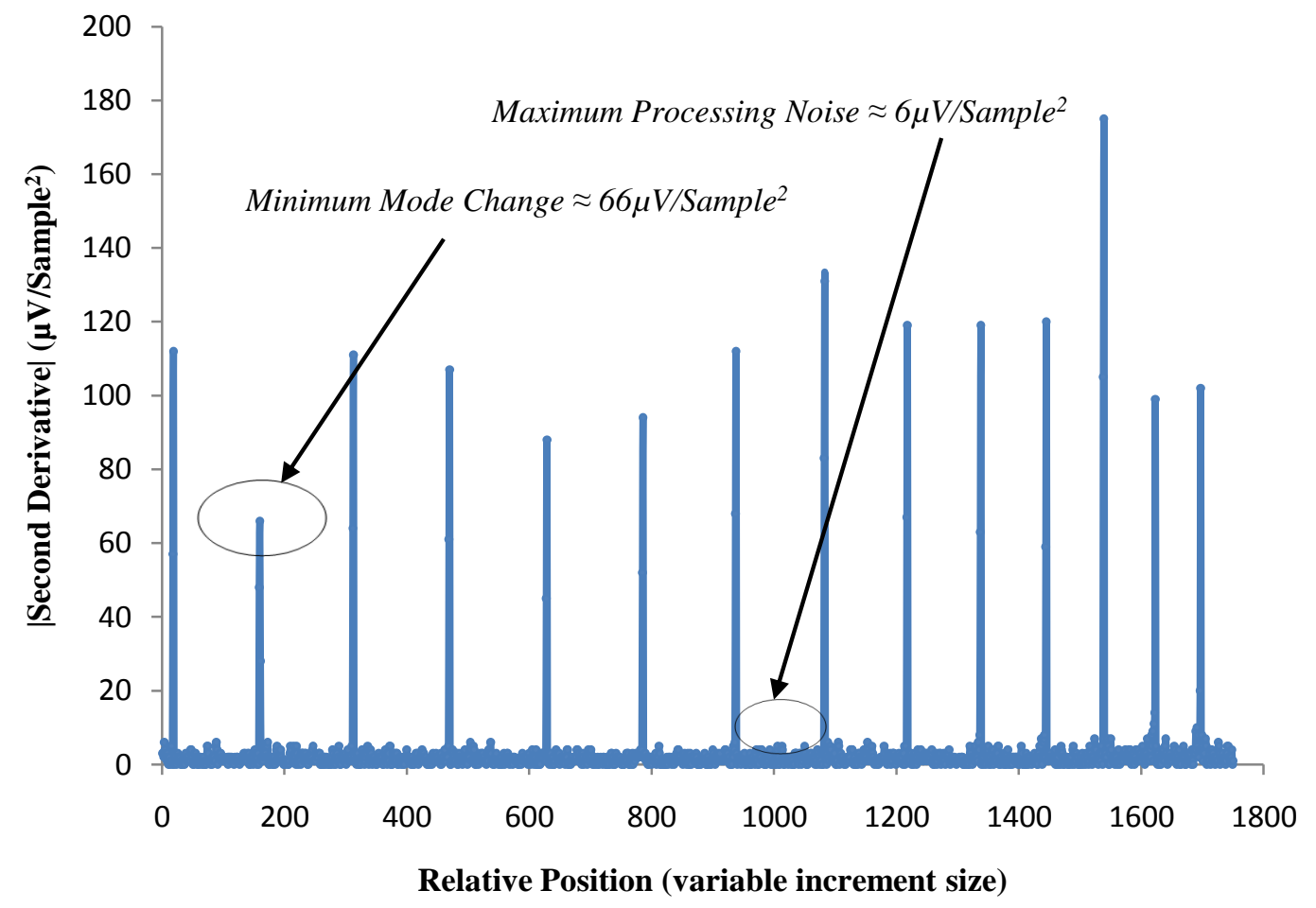

Figure 4.12: Absolute value of second derivative voltage response for C-Band path 5 
The first and second order voltage derivatives are analyzed to better understand how to sense the instantaneous voltage changes. Figures (4.3-4.8) demonstrate the LBand characteristic with a constant increment size (1001 points) along the paths, whereas figures (4.9-4.12) demonstrate the C-Band characteristic with a variable increment size along the paths based on equation (4.2). A variable step size reduces the possibility of skipping a mode change and also reduces the noise in the second derivative response.

$$
\Delta V=\frac{F M(V) B M(V)}{F M(V)_{M A X} B M(V)_{M A X}}
$$

As seen in the gain medium voltage characteristics, an appropriate threshold level can be chosen to sense a wavelength mode change. However, to enable a fully automated calibration, the actual threshold value itself must be calculated internally. This is possible through equation (4.3) observed from the data depicted in tables (4.1-4.2).

$$
\text { Path Threshold } \stackrel{\text { def }}{=} \bar{x}+2 \sigma_{x}+1.0 \mu V
$$

It is seen in figures (4.13) and table (4.1) that a constant increment size along a path doesn't accurately produce reliable threshold values. Therefore, a second derivative voltage sensing procedure with a variable increment size was chosen which enhances the sharp transitions, reduces the noise and fully predicts mode transitions for all paths for any tested SG-DBR laser. It was observed for the C-Band that a sufficient threshold level can be calculated by performing a statistical analysis of the second derivative voltage sweep. A threshold which is two standard deviations away from the mean accurately falls within the maximum noise value and minimum detectable wavelength mode change as described in expression (4.4). An additional $1.0 \mu \mathrm{V} /$ Sample $^{2}$ is also added to the calculated threshold to provide a slight room for error.

$$
\text { Maximum Noise < Threshold < Minimum Mode Change }
$$


Table 4.1: Summary of calculated threshold and corresponding ranges for L-Band with a constant increment size along paths

\begin{tabular}{|c|c|c|c|c|c|c|c|c|}
\hline $\begin{array}{c}\mathrm{L}-\text { Band } \\
\left(\mu \mathrm{V} / \text { Sample }^{2}\right) \\
\end{array}$ & $\begin{array}{c}\text { Path } \\
1 \\
\end{array}$ & $\begin{array}{c}\text { Path } \\
2 \\
\end{array}$ & $\begin{array}{c}\text { Path } \\
\mathbf{3} \\
\end{array}$ & $\begin{array}{c}\text { Path } \\
4 \\
\end{array}$ & $\begin{array}{c}\text { Path } \\
5 \\
\end{array}$ & $\begin{array}{c}\text { Path } \\
6\end{array}$ & $\begin{array}{c}\text { Path } \\
7 \\
\end{array}$ & $\begin{array}{c}\text { Path } \\
8 \\
\end{array}$ \\
\hline Max Noise & 50 & 50 & 60 & 50 & 50 & 50 & 50 & 50 \\
\hline Min Mode Change & 70 & 100 & 100 & 190 & 200 & 140 & 100 & 50 \\
\hline Average & 15.96 & 16.4 & 15.23 & 17.02 & 16.9 & 15.78 & 14.10 & 13.36 \\
\hline Standard Deviation & 25.08 & 26.37 & 24.92 & 35.71 & 34.04 & 22.89 & 14.50 & 11.70 \\
\hline Threshold & 67.12 & 70.15 & 66.06 & 89.42 & 85.98 & 62.60 & 44.20 & 37.77 \\
\hline
\end{tabular}

Table 4.2: Summary of calculated threshold and corresponding ranges for C-Band with a variable increment size along paths

\begin{tabular}{c|c|c|c|c|c|c|c} 
C- Band $\left(\boldsymbol{\mu V} /\right.$ Sample $\left.^{2}\right)$ & Path 1 & Path 2 & Path 3 & Path 4 & Path 5 & Path 6 & Path 7 \\
\hline \hline Max Noise & 10 & 8 & 11 & 7 & 6 & 15 & 7 \\
Min Mode Change & 13 & 29 & 58 & 76 & 66 & 74 & 45 \\
Average & 2.35 & 2.51 & 2.84 & 3.22 & 3.07 & 2.78 & 2.60 \\
Standard Deviation & 4.24 & 6.71 & 10.07 & 13.44 & 11.39 & 8.47 & 6.31 \\
\hline Threshold & $\mathbf{1 1 . 8 2}$ & $\mathbf{1 6 . 9 2}$ & $\mathbf{2 3 . 9 7}$ & $\mathbf{3 1 . 1 0}$ & $\mathbf{2 6 . 8 5}$ & $\mathbf{2 0 . 7 3}$ & $\mathbf{1 6 . 2 2}$
\end{tabular}

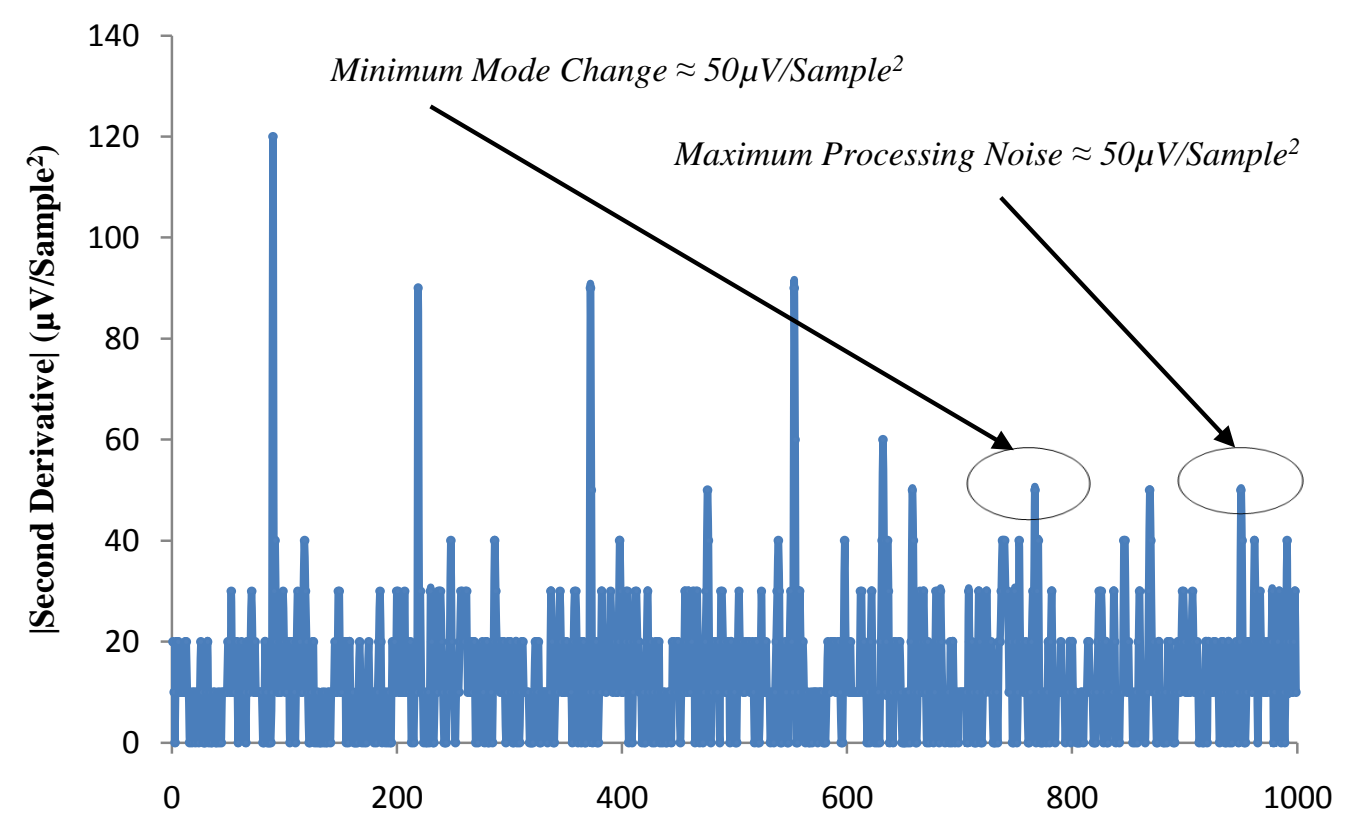

Relative Position (999 data points, constant increment size)

Figure 4.13: Absolute value of second derivative voltage response for $L$-Band path 8 where the wavelength mode transitions are indistinguishable from processing noise 


\subsection{1 - Hysteresis Characteristics}

The two-dimensional voltage response characteristics within section (4.2) provide the needed derivative threshold information to allow for automated calibration. However, it's necessary to investigate the hysteresis characteristics along a path. Figure (4.14) depicts how the voltage response within a path retains relatively the same magnitude regardless of the direction of progression. This feature simplifies the sensing procedure by enabling the use of an absolute threshold value based on the internally calculated path specific threshold.

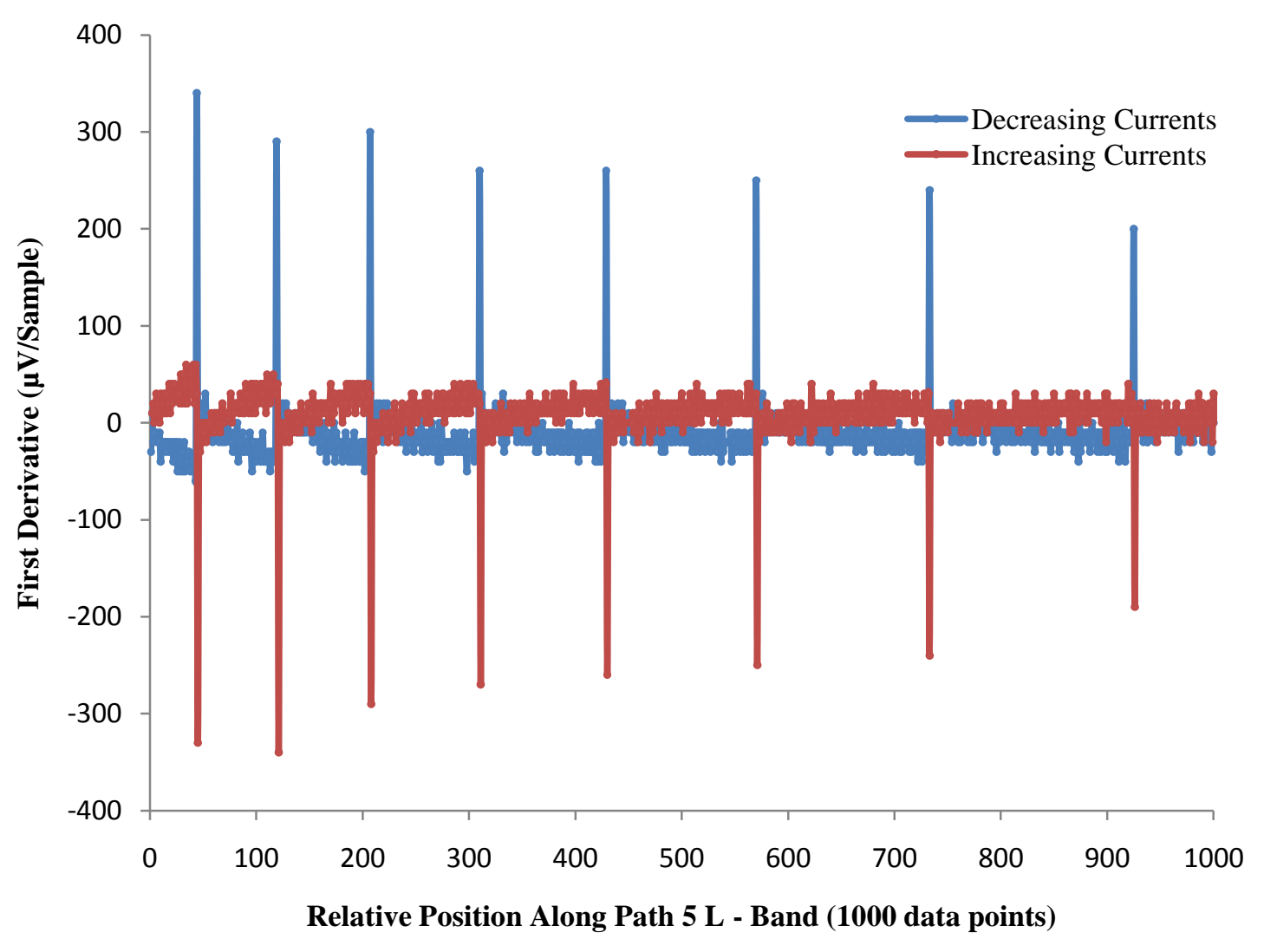

Figure 4.14: Hysteresis characteristics for two-dimensional mirror tuning 


\subsection{2 - SMSR Comparison}

As previously discussed in subsections (3.8.2 and 3.8.3) the optimal tuning path is characterized at the center of the tuning pads. To further verify this phenomenon, the SMSR for normal and fine resolution is plotted with the voltage response against the front mirror current along a linear two-dimensional progression of a wavelength pad. The following figures demonstrate that the center of the tuning pad is in fact the optimal area for the side mode suppression as expected. It's also important to note that the previous normal resolution SMSR data wasn't taken over a full pad as depicted in figure (4.15). These plots further exemplify the advantage of utilizing the gain medium voltage response as a wavelength pad sensing measurement.

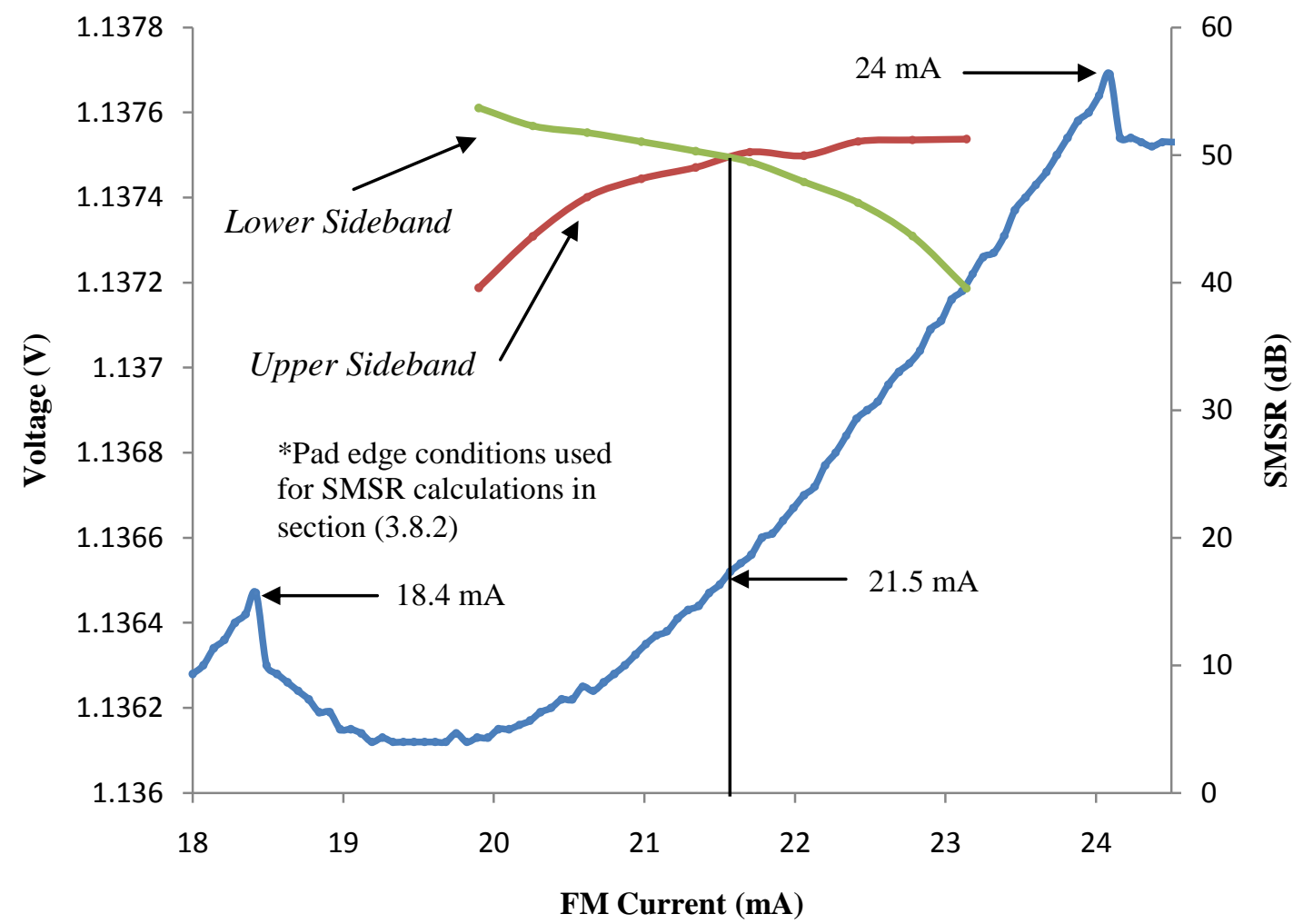

Figure 4.15: SMSR related to the voltage response across a single tuning pad. Equal SMSR occurs at the middle of the pad (path 5, L-Band) when the front mirror current is approximately $21.5 \mathrm{~mA}$. 


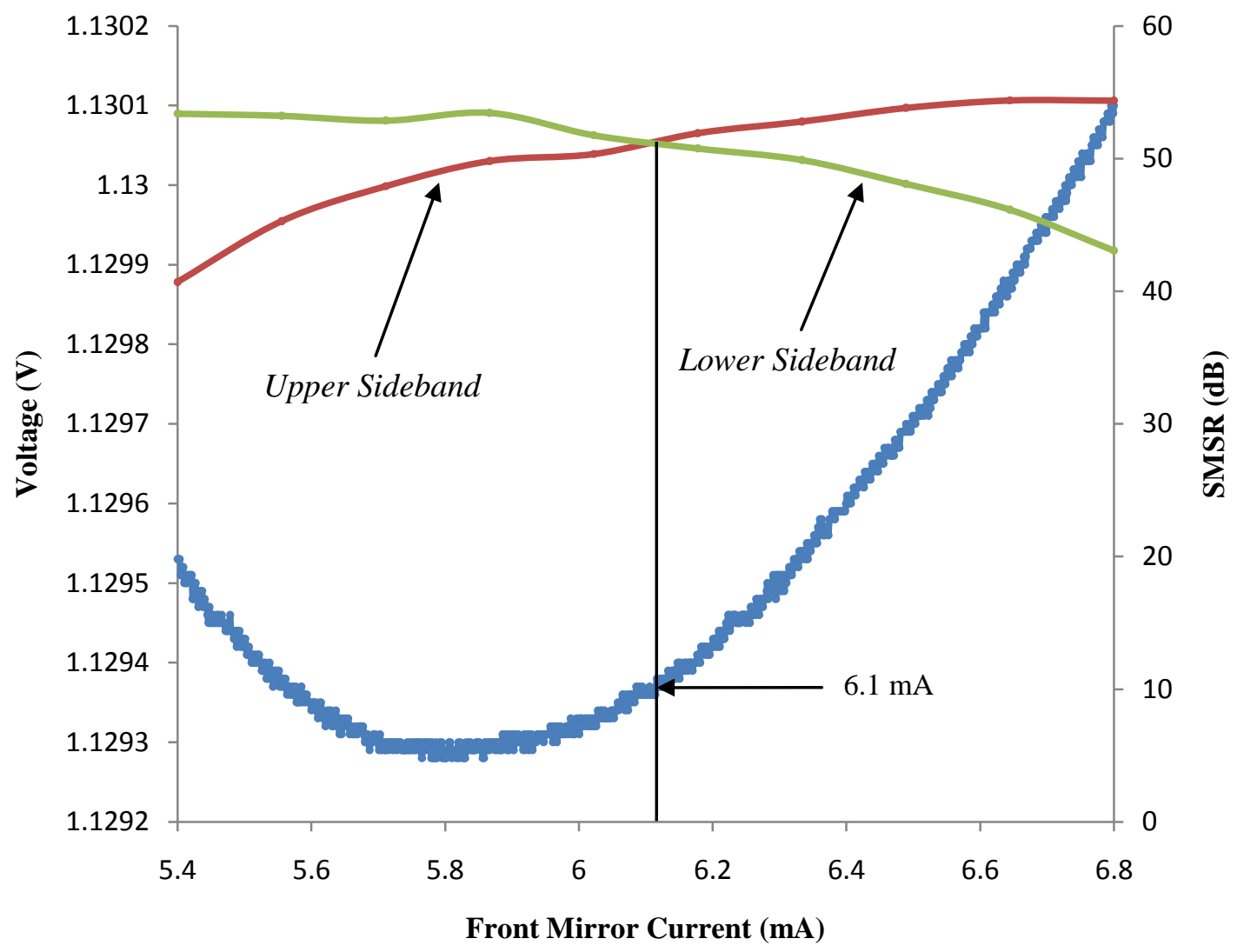

Figure 4.16: SMSR related to the voltage response across a single tuning pad. Equal SMSR occurs at the middle of the pad (path 5, L-Band, fine resolution) when the front mirror current is approximately $6.1 \mathrm{~mA}$. 


\section{3 - Three-Dimensional Characterization}

The three-dimensional characterization of the gain medium voltage response was qualitatively conducted to gain a better understanding of the wavelength pad propagation. In the same way that the previous voltage responses were measured, a LabVIEW program was written to sweep over all possible three-dimensional current mirror combinations. The phase mirror current is initialized to $0 \mathrm{~mA}$ while the front mirror current sweeps from $0 \mathrm{~mA}$ to $35 \mathrm{~mA}$ as the back mirror current also increments from 0 $\mathrm{mA}$ to $60 \mathrm{~mA}$. Once each current mirror combination voltage is recorded with that particular phase mirror current, the phase mirror current is slightly incremented. Once the phase mirror current reaches $20 \mathrm{~mA}$, the program ends. Figures (4.17 and 4.18) on the following pages show how the gain medium voltage response is related to the wavelength mode map whereas figures (4.19-4.22) demonstrate how the phase mirror current is directly related to wavelength pad movement. This three-dimensional feature will be implemented and discussed in greater detail in chapter 5. 


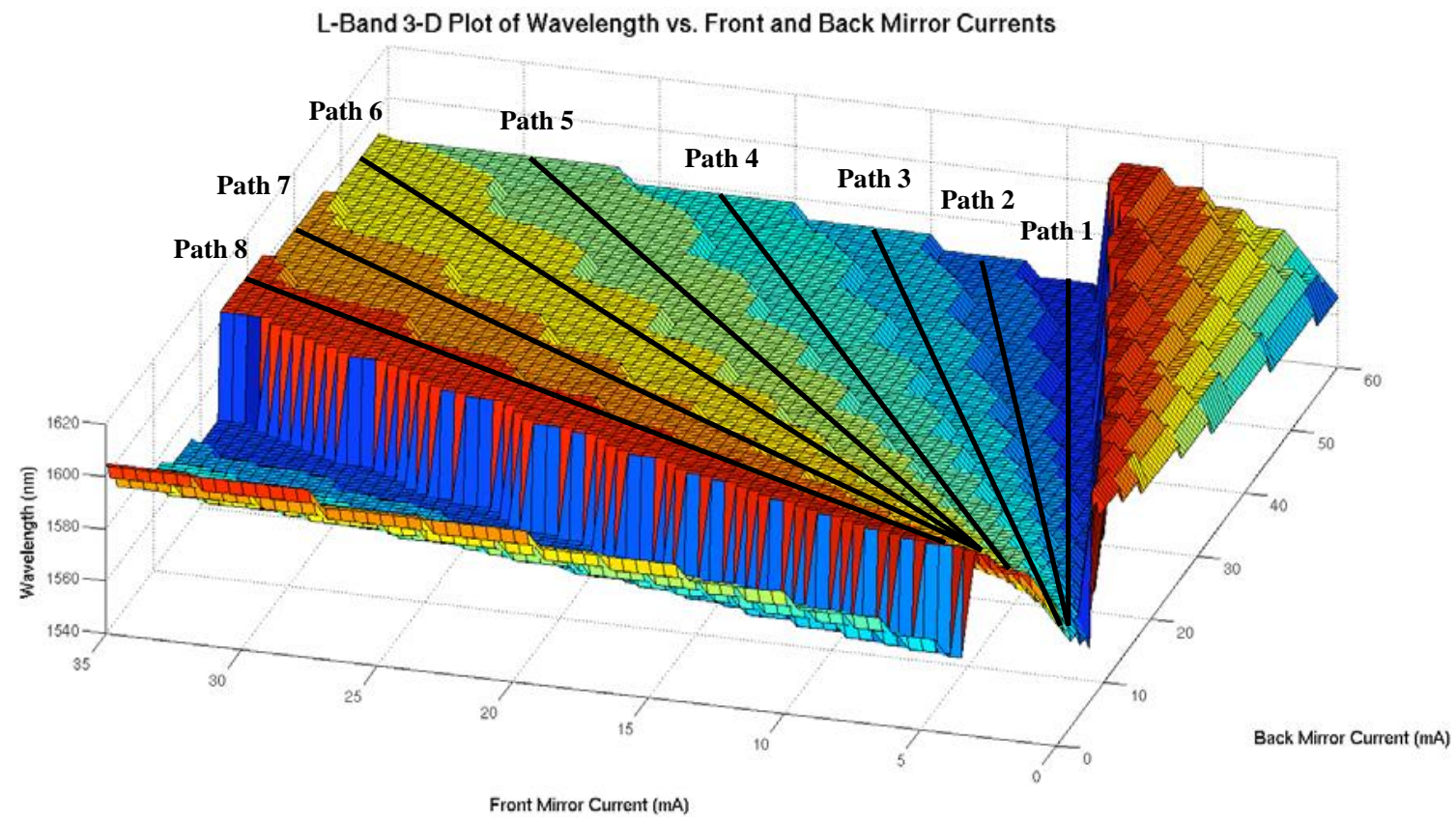

Figure 4.17: Three-dimensional (mode map) plot of wavelength vs. mirror currents for the L-band laser [15]

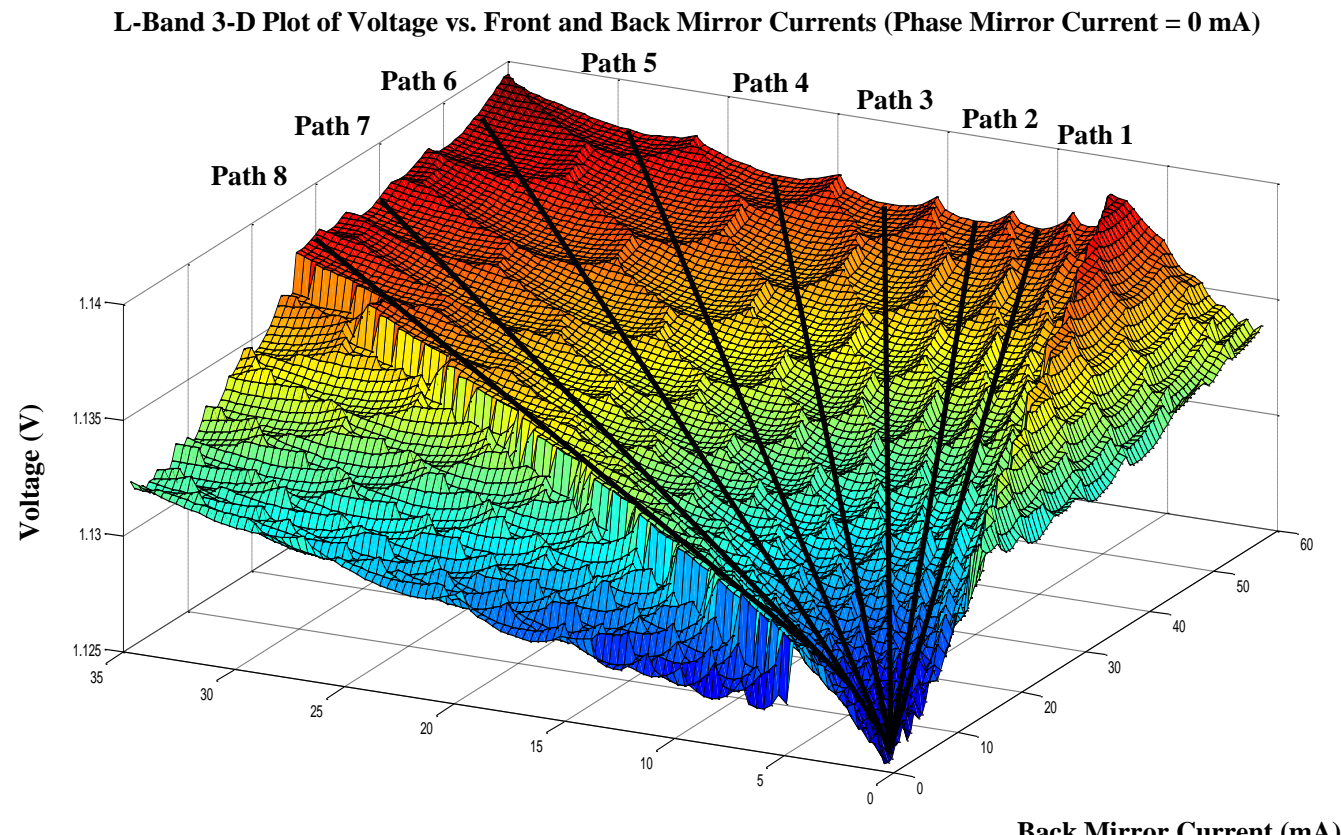

Front Mirror Current (mA)

Figure 4.18: Three-dimensional (mode map) plot of voltage vs. mirror currents (phase mirror current $=0 \mathrm{~mA}$ ) for the $\mathrm{L}$-band laser. 
L-Band 3-D Plot of Voltage vs. Front and Back Mirror Currents (Phase Mirror Current $=5 \mathrm{~mA}$ )

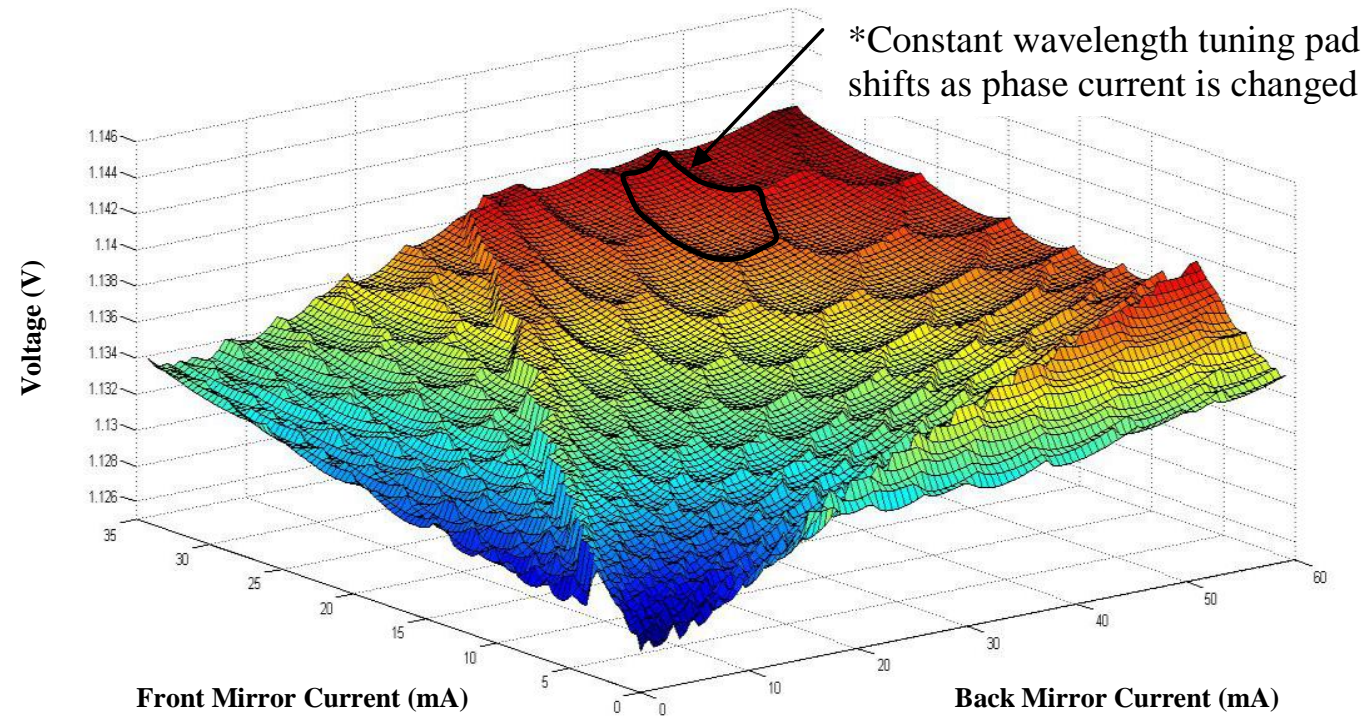

Figure 4.19: Three-dimensional (mode map) plot of voltage vs. mirror currents (phase mirror current $=5 \mathrm{~mA}$ ) for the $L$-band laser.

L-Band 3-D Plot of Voltage vs. Front and Back Mirror Currents (Phase Mirror Current $=10 \mathrm{~mA}$ )

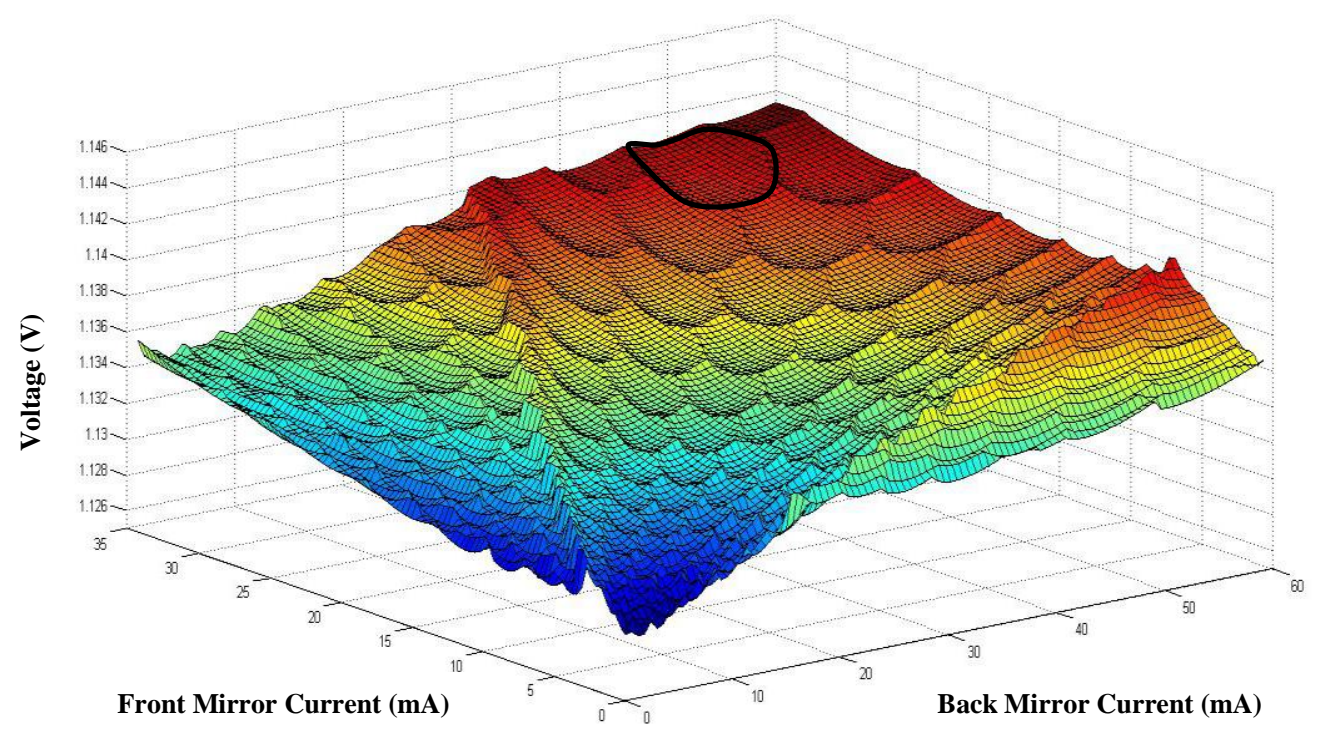

Figure 4.20: Three-dimensional (mode map) plot of voltage vs. mirror currents (phase mirror current $=10 \mathrm{~mA}$ ) for the $L$-band laser. 
L-Band 3-D Plot of Voltage vs. Front and Back Mirror Currents (Phase Mirror Current $=15 \mathrm{~mA}$ )

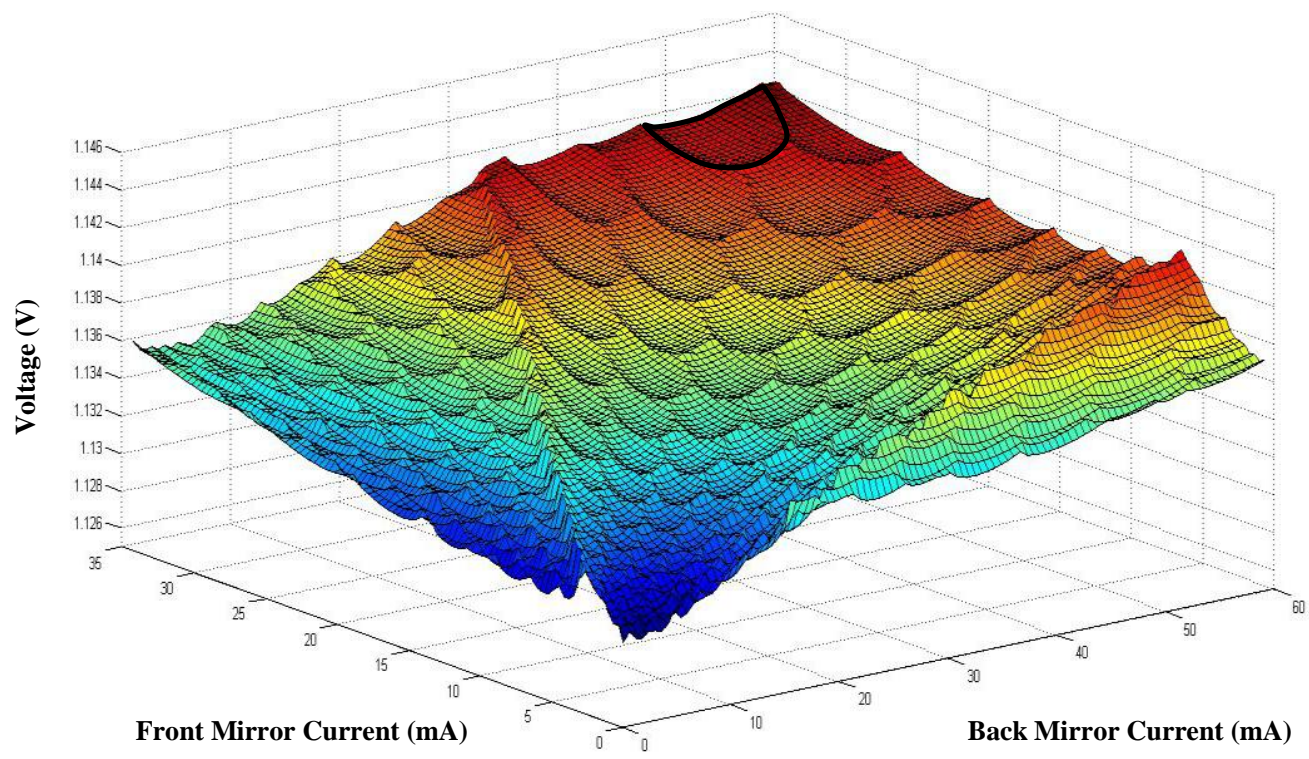

Figure 4.21: Three-dimensional (mode map) plot of voltage vs. mirror currents (phase mirror current $=15 \mathrm{~mA}$ ) for the $L$-band laser.

L-Band 3-D Plot of Voltage vs. Front and Back Mirror Currents (Phase Mirror Current $=20 \mathrm{~mA}$ )

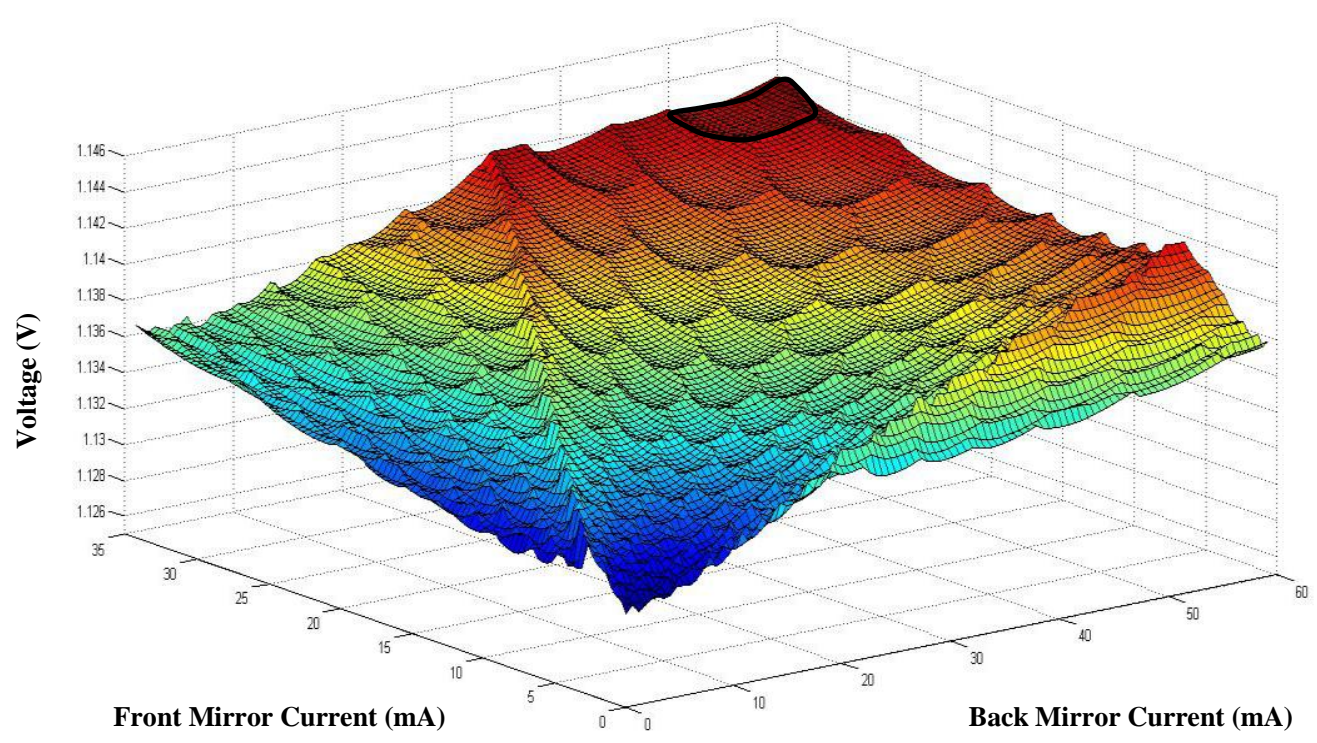

Figure 4.22: Three-dimensional (mode map) plot of voltage vs. mirror currents (phase mirror current $=20 \mathrm{~mA}$ ) for the $L$-band laser. 


\section{CHAPTER 5 -Automated Laser Calibration}

The previous chapters provide the foundation for creating an automated laser calibration procedure. Throughout this chapter the implemented algorithm and calibration results are presented. The core functionality of this program depends largely on the second derivative threshold sensing discussed in chapter 4. The most straight forward solution with an automated OSA based calibration approach would be too timeconsuming; therefore, a more efficient measurement methodology is implemented with the gain medium voltage. The objective in this process is to create a fully automated laser calibration program which produces the necessary synchronized input waveforms to produce a linear frequency ramp over time. The automated process should dramatically reduce calibration time, increase bandwidth, and improve power leveling, when compared to the previous manual efforts.

A high level algorithm will be outlined as well as specific low level program functionality, in particular how to locate paths and centers of wavelength tuning pads. An implemented solution for pad edge cases is provided as well as the data processing methodology. The calibration results which include the previously mentioned program expectations are also presented.

\section{1 - Outline of Algorithm}

The automated calibration program is written in Python to allow for communication with the measurement instruments through a General Purpose Interface Bus (GPIB - IEEE 488). The program begins with the graphical user interface (GUI) depicted in figure (5.2). This interface allows the user to connect to the specific instruments displayed in figure (5.1), which enables the program to be location and 
device adaptive as shown in figure (5.3). It also has an initial "Laser Type" dropdown choice shown in figure (5.4) with initialization values based on the example settings file depicted in figure (5.5). This feature allows the user to add new lasers as shown in figures (5.6-5.7). It's important to note that the only part of the calibration program that is specific to the laser of interest is within the settings file. This enables a completely automated calibration process regardless of the laser type.

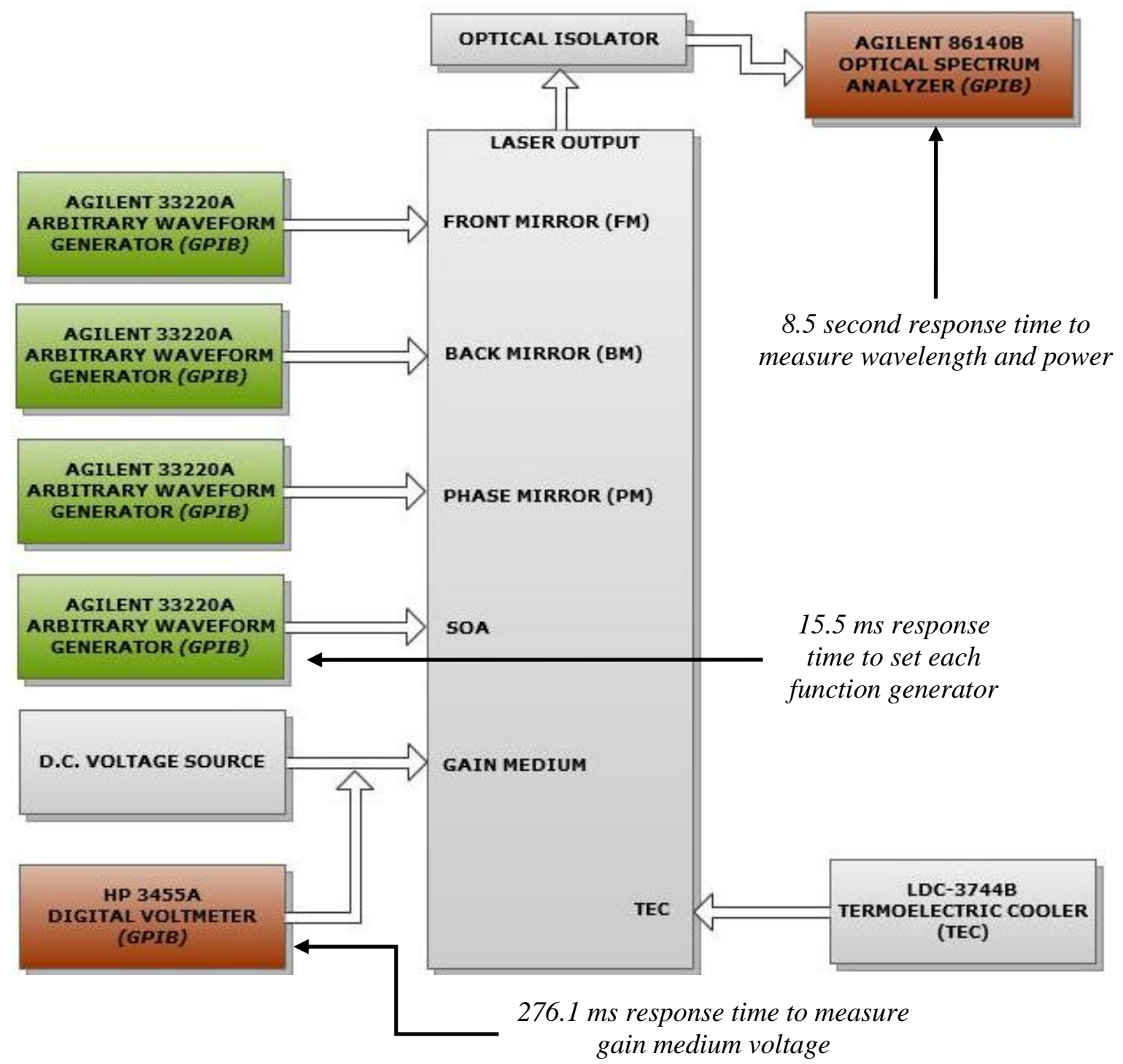

Figure 5.1: Block diagram of automated calibration instrumentation setup 


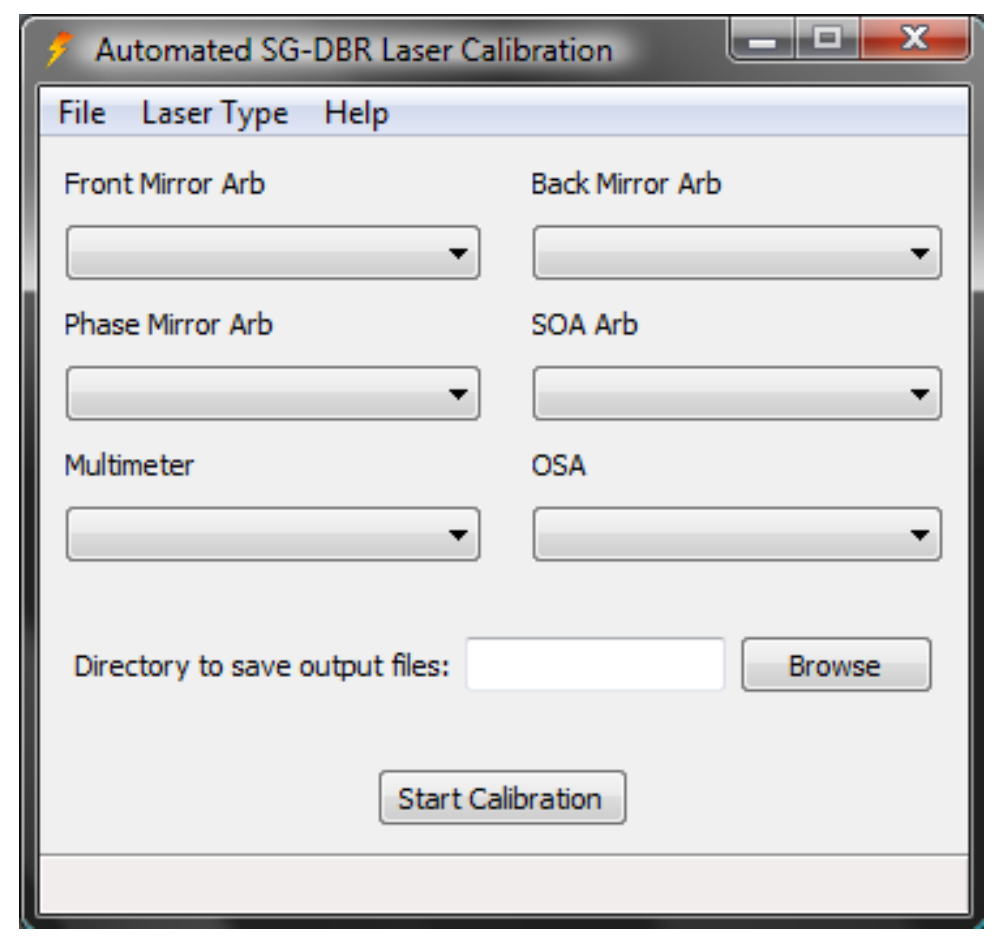

Figure 5.2: Front panel GUI at program initialization

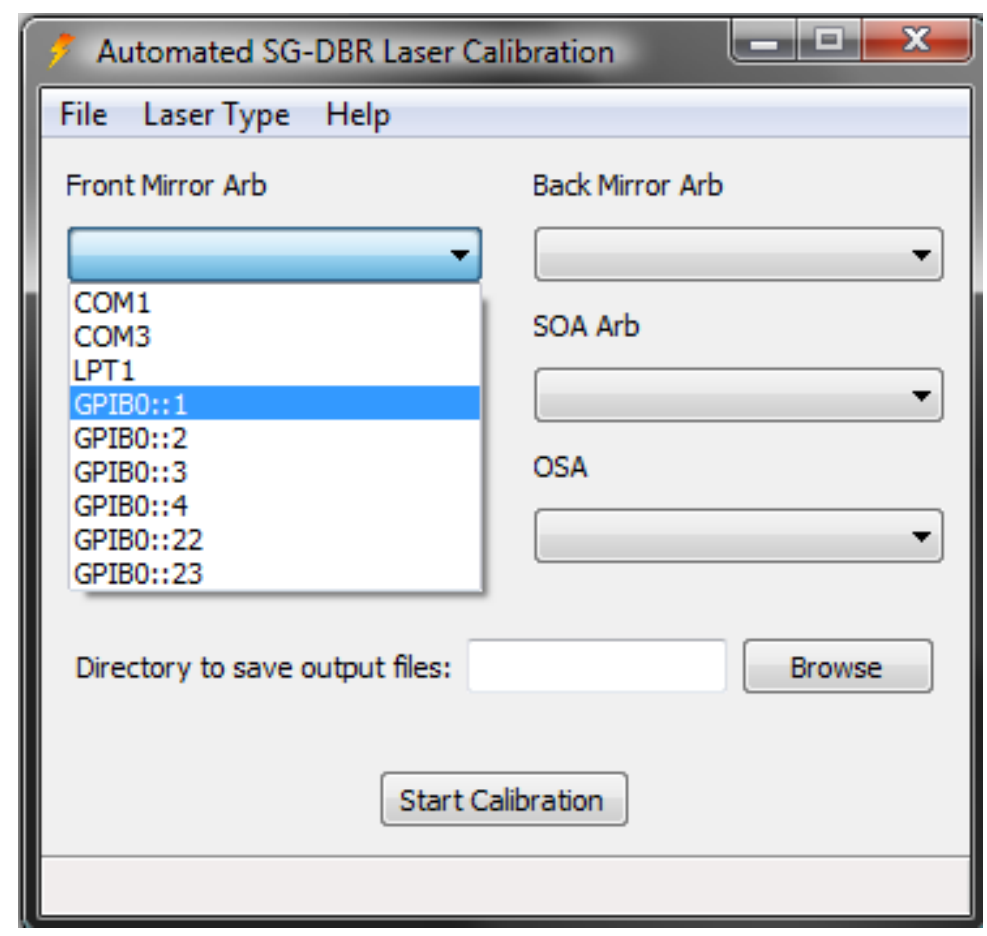

Figure 5.3: Front panel GUI with device address dropdown choices 


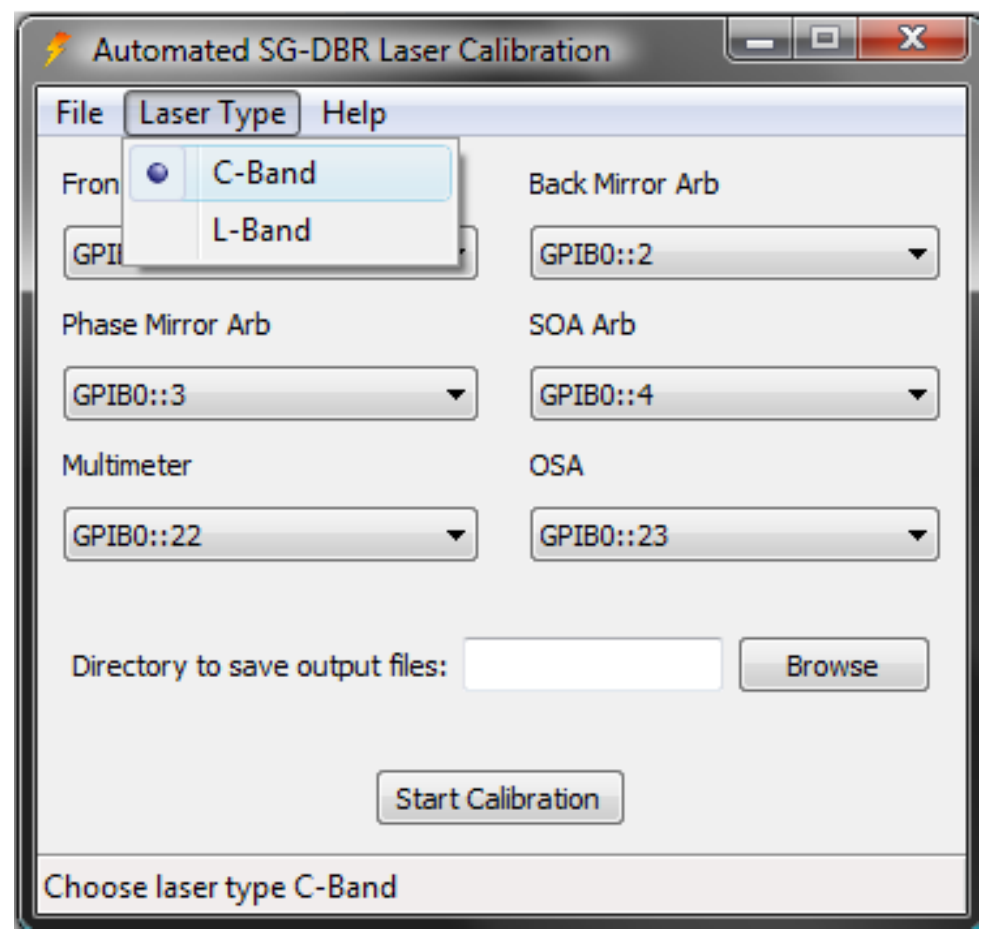

Figure 5.4: Front panel GUI with laser type dropdown choices

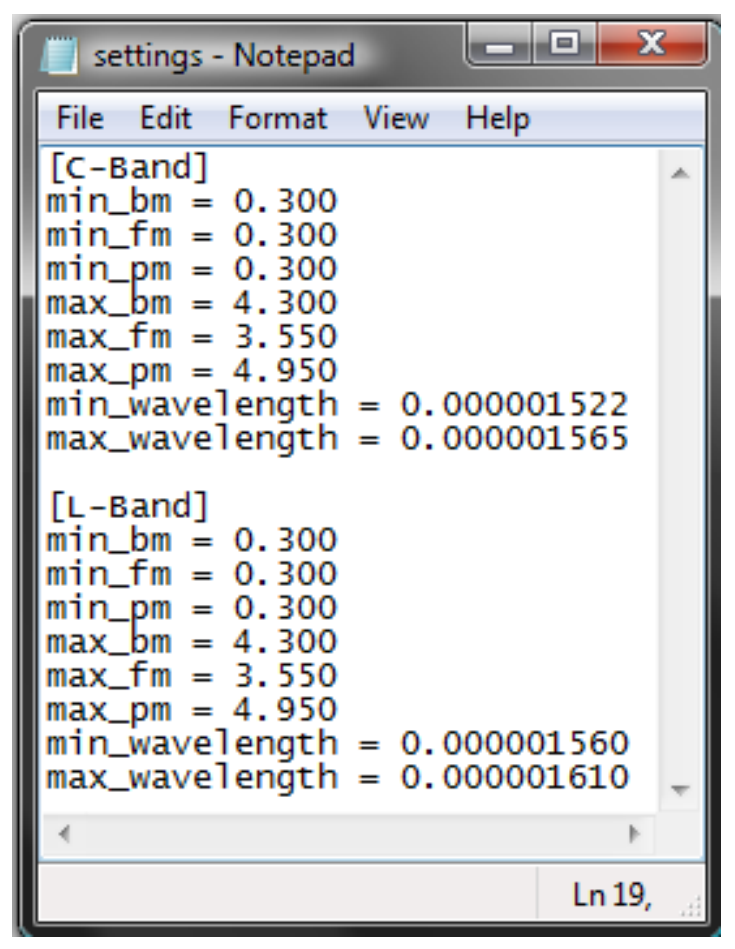

Figure 5.5: Laser type settings file corresponding to the choices in figure (5.4) 


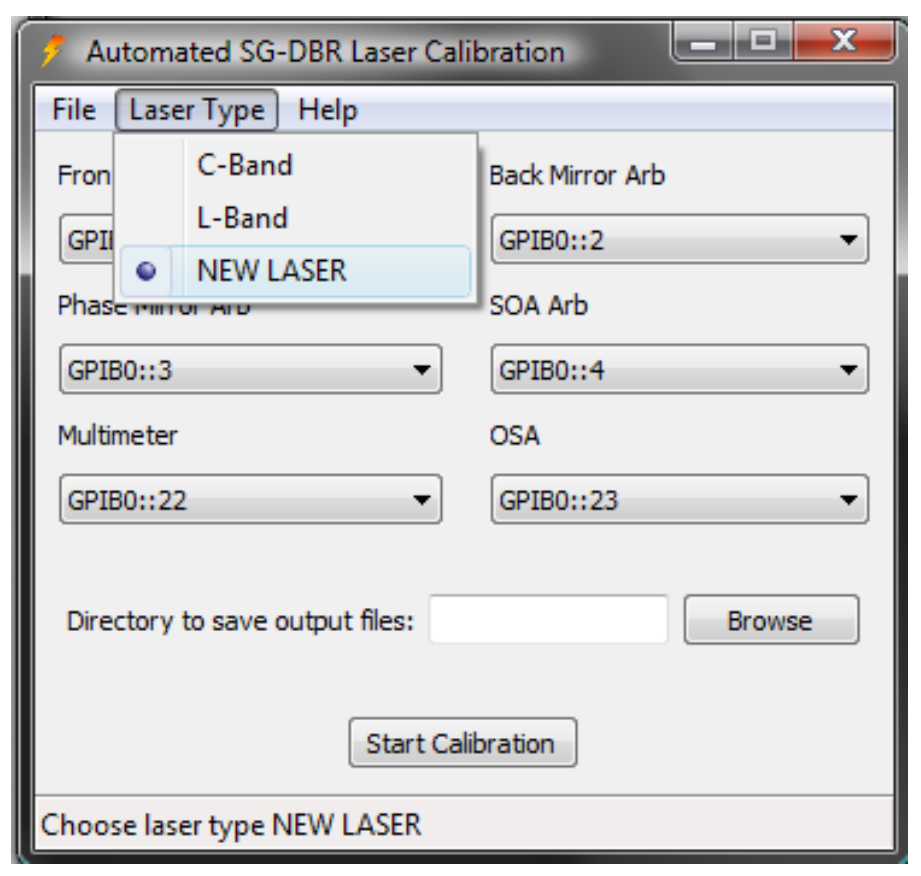

Figure 5.6: Front panel GUI with additional laser type dropdown choice

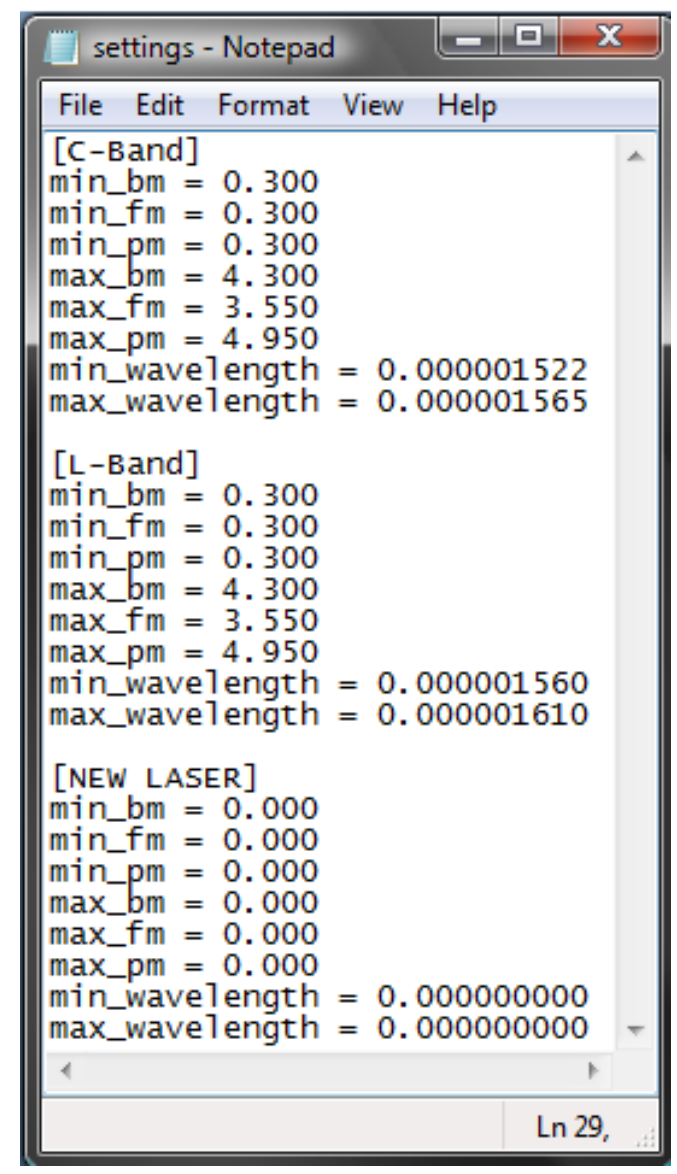

Figure 5.7: Laser type settings file corresponding to the new choice in figure (5.6) 
Once the directory for the output files is chosen and the "Start Calibration" button is pressed, the program obtains the device addresses and settings information specified in the setup process. After device initialization, start and stop points for all tunable paths are located. With these points, the path slope is calculated and used to collect the second derivative gain medium voltage for the entire path as seen in chapter 4 . The appropriate path specific threshold is calculated based on the internal statistical analysis of that data. At this point, enough information regarding the path of interest has been obtained to begin traversing the path for wavelength pad centers. Once traversal has completed, the process is repeated for all remaining paths. The program finishes by stitching and power leveling the linear frequency interpolated data and returning the synchronized waveform data to the directory specified by the user. Figure (5.8) provides a high level flowchart of the entire automated calibration process. 


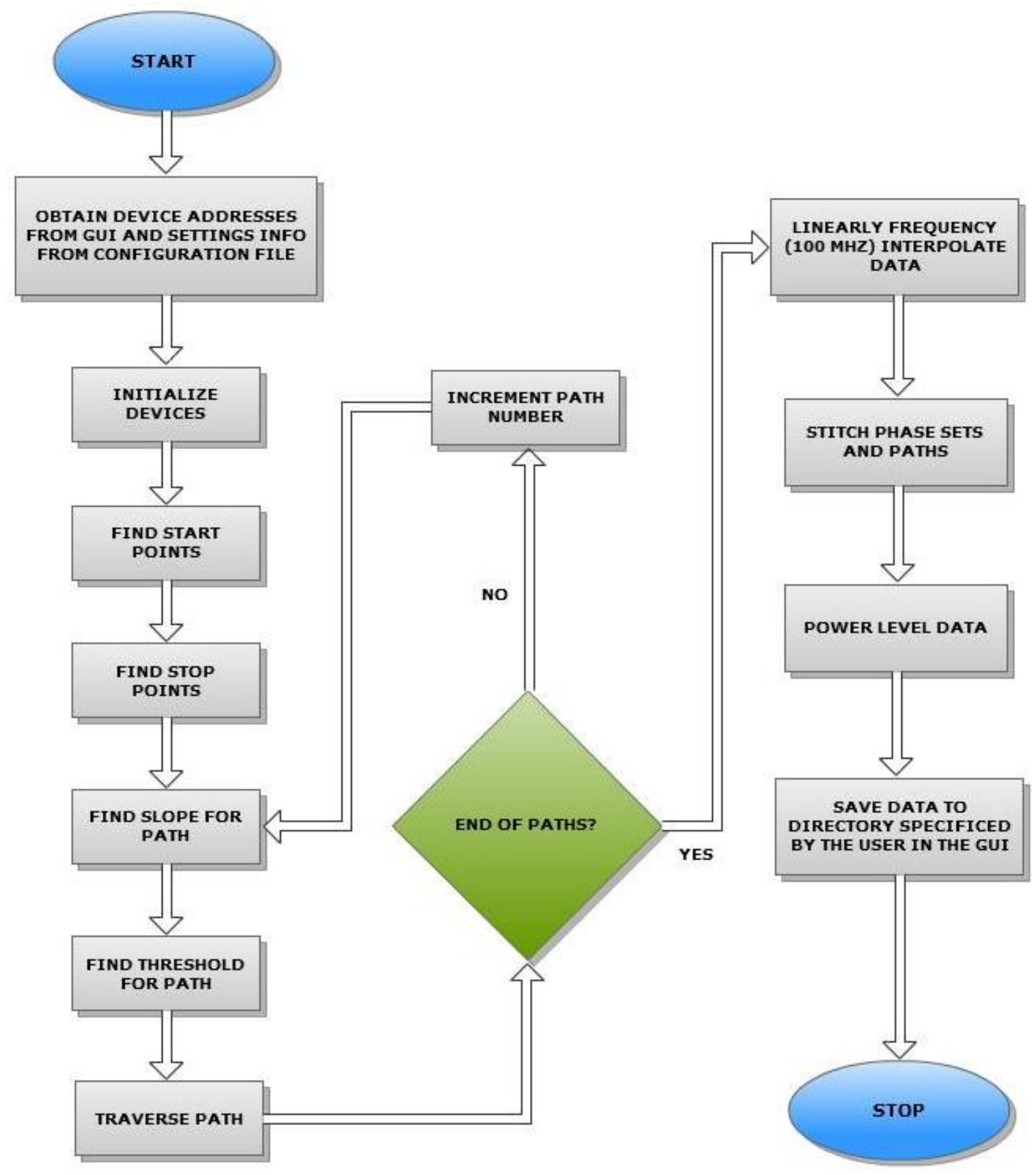

Figure 5.8: High level software flowchart for the automated calibration process 


\section{2 - Locating Start and Stop Path Points}

Locating the beginning and ends of paths is extremely important to the automation procedure. To begin locating the start points of paths, the function initializes to the minimum back mirror (BM) and front mirror (FM) voltage values ("min_bm" and "min_fm") according to the settings file. As seen in figure (5.9) by the arrows, BM is increased while FM is kept constant. The wavelength is continuously recorded by the OSA to sense a $5 \mathrm{~nm}$ mode hop. Figure (5.10) demonstrates this process in a low level software flowchart. Each set of path edge pairs are averaged to return the path start point until the last path wavelength ( $3 \mathrm{~nm}+$ "min_wavelength") is sensed. This process is repeated with FM increasing and BM kept constant until the maximum wavelength is sensed (no additional tunable paths exist).

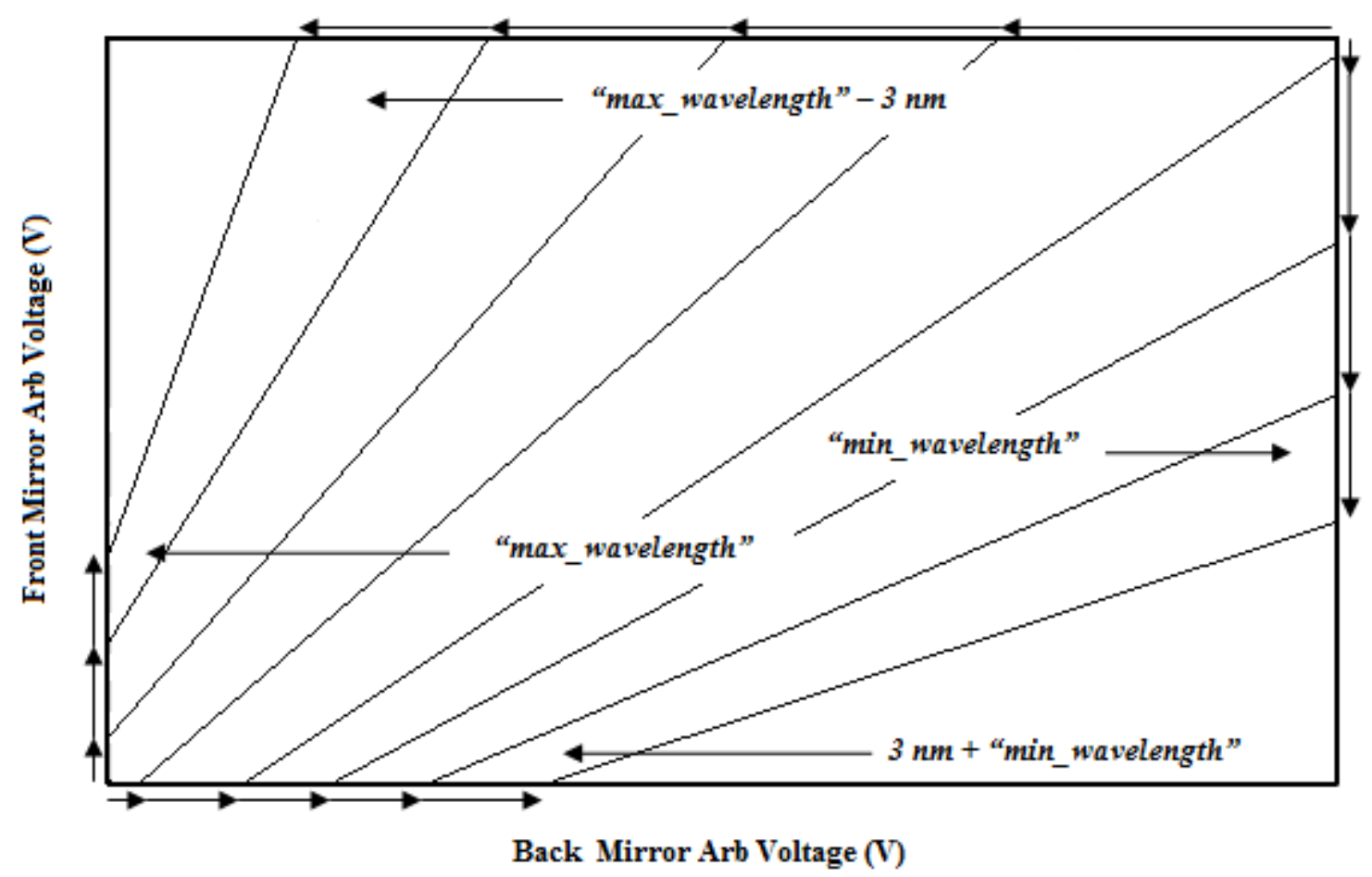

Figure 5.9: Visual representation of locating the start and stop points of paths 
This same algorithm is implemented for locating the ends of paths with slight initialization and sensing differences as seen in the flowchart differences in figures (5.115.12). Rather than incrementing $\mathrm{BM}$ and $\mathrm{FM}$, the function initializes the values to "max_bm" and "max_fm" and searches for pad edges by decreasing the position. It's important to note that an OSA wavelength sensing procedure is necessary to insure extremely accurate start and stop values as opposed to a second derivative onedimensional voltage threshold. If the beginning or end values of a path and subsequently the path slope is slightly off, then the calibration for that particular path won't be as accurate.

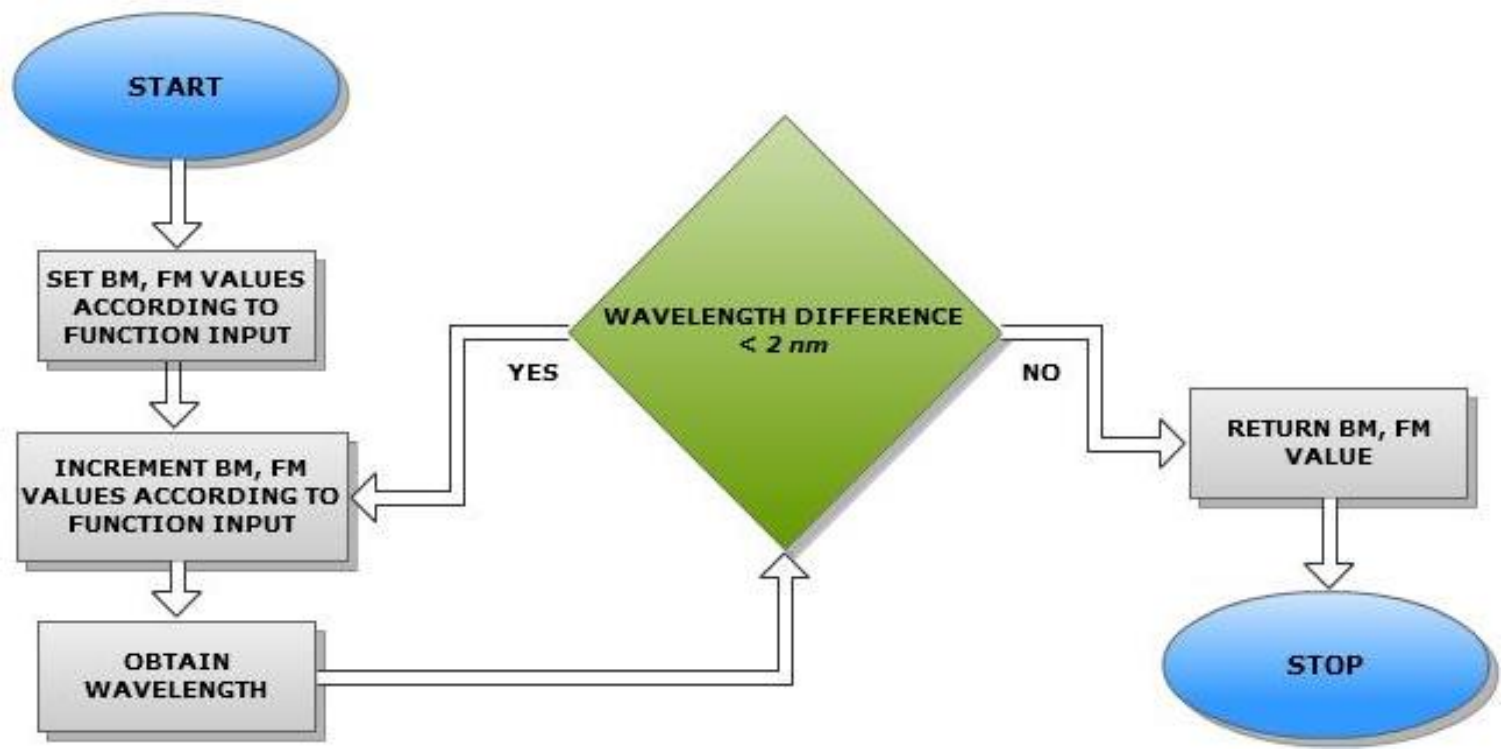

Figure 5.10: Low level software flowchart for path edge detection 


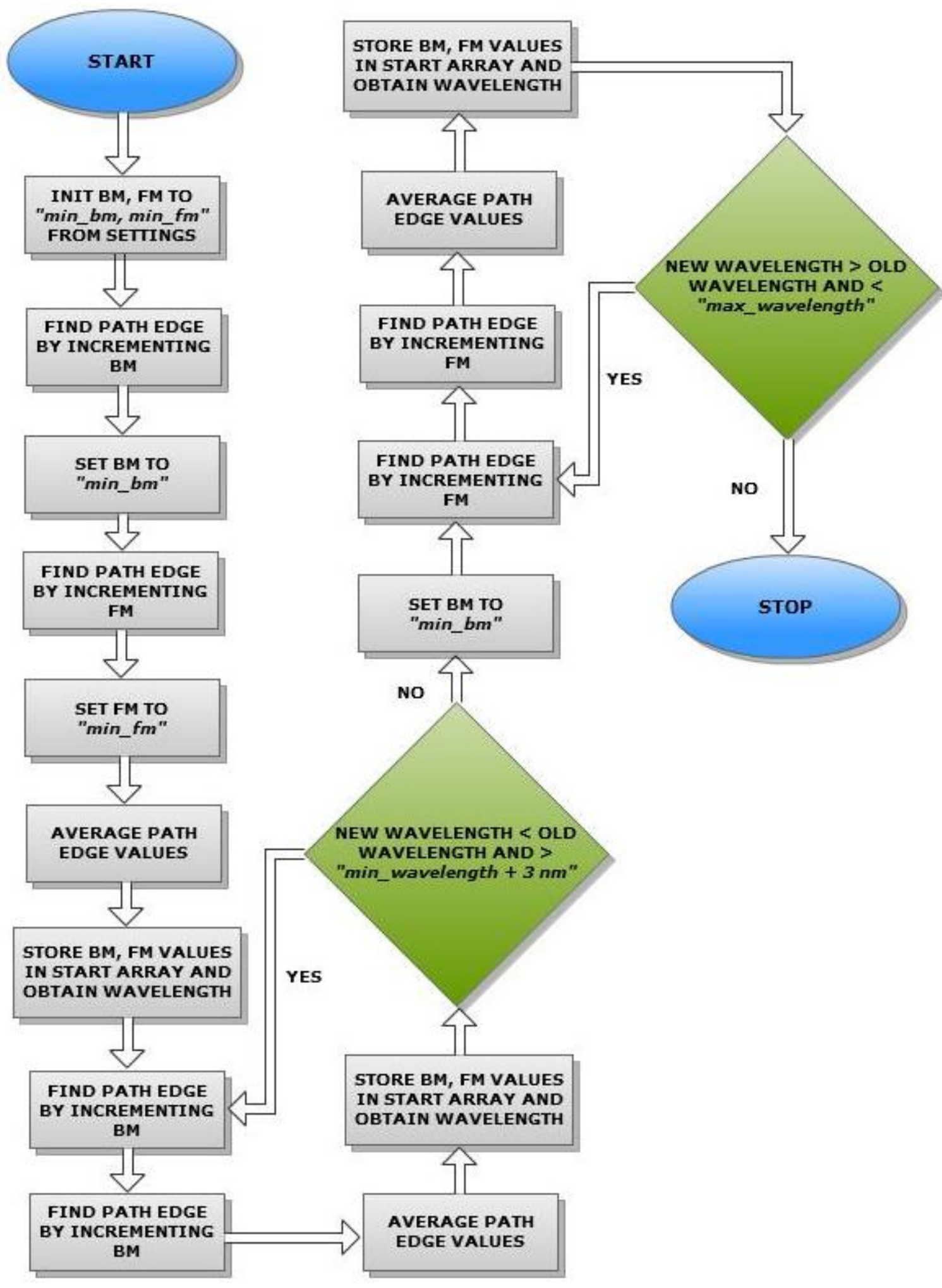

Figure 5.11: Software flowchart for locating the start points of a path 


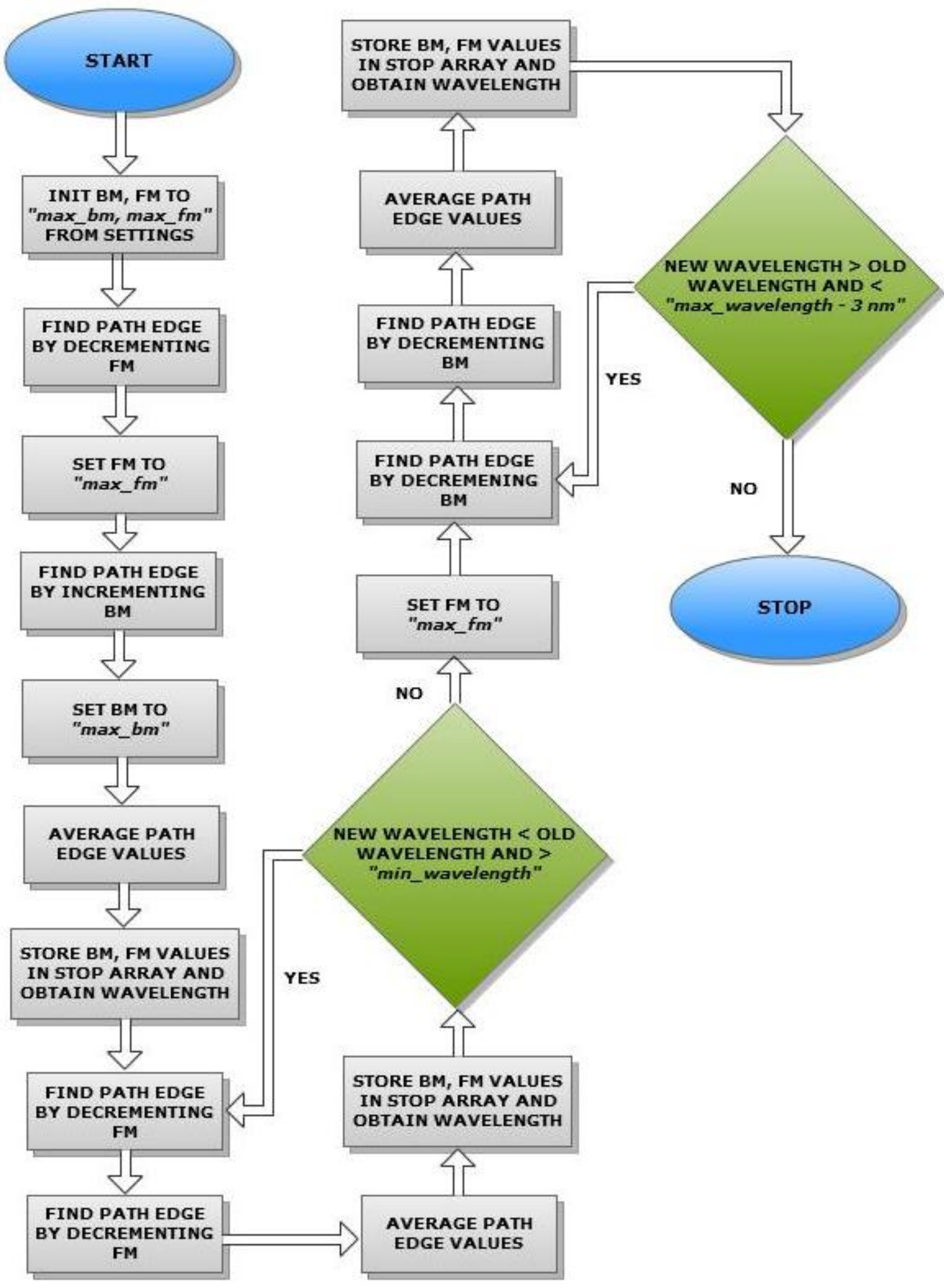

Figure 5.12: Software flowchart for locating the stop points of a path 


\section{3 - Traversing a Path}

Once the path locations and specific path slope is determined, the path specific threshold is found before traversing a path for wavelength pad centers. As previously mentioned, chapter 4 provided the proof of concept of finding a path specific threshold and is implemented in software demonstrated by figure (5.13). The gain medium voltage over the entire path is recorded until either BM or FM reaches "min_bm" or "min_fm" respectively. The second derivative threshold is then calculated and recorded for the path.

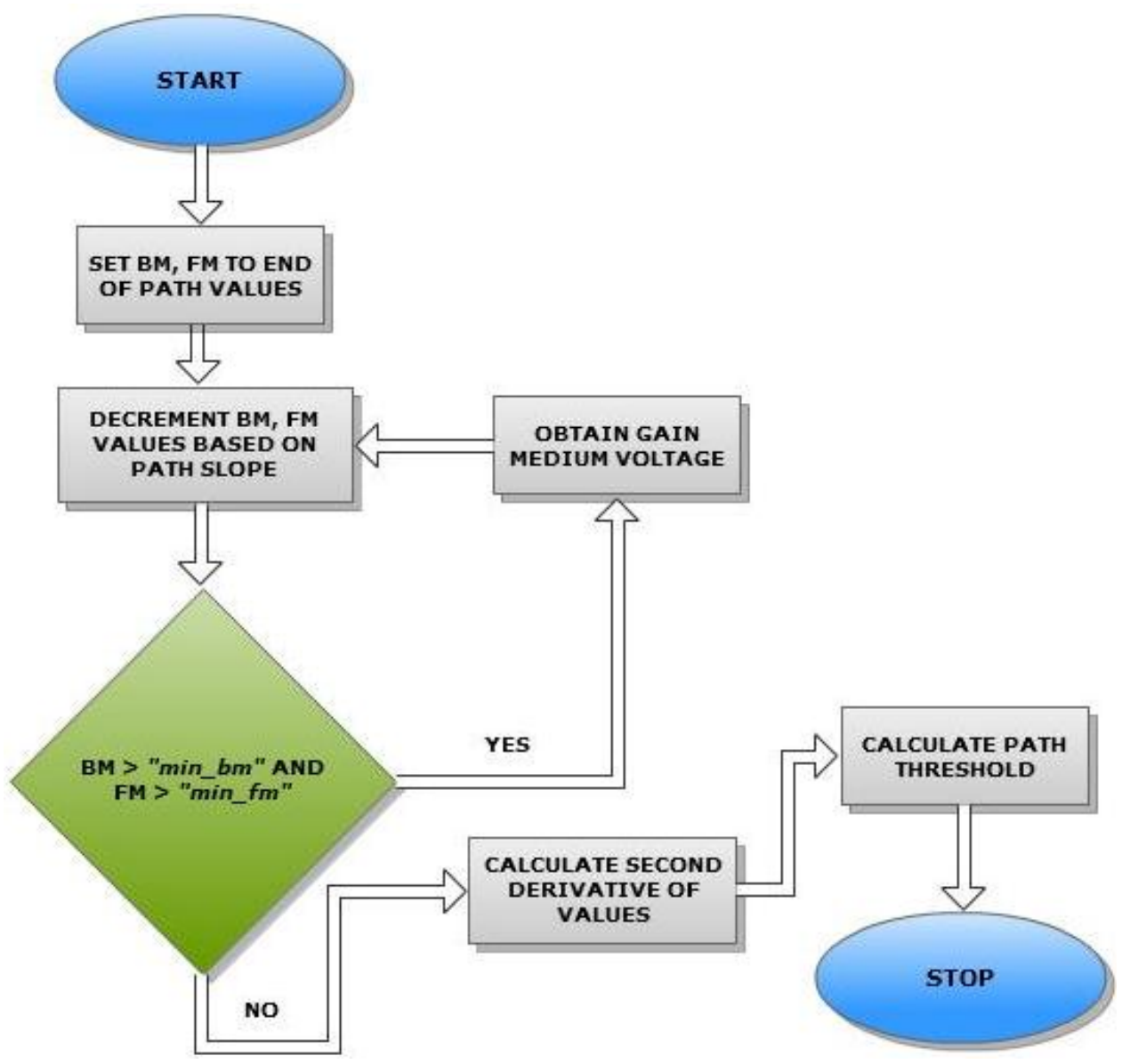

Figure 5.13: Software flowchart for threshold calculation 
After the path location, slope, and threshold values are found for the current path, the program begins to traverse the path to search for the wavelength pad centers. The traverse path function is initialized with $\mathrm{BM}$ and FM set to the start points. The values are incremented based on the path slope until a mode change is detected, which is described in figure (5.14). The values are set to a projected center based on the previous center and the mode change value (derivative peak) by equation (5.1).

$$
\text { projected }=\text { previous }+\frac{\text { peak-previous }}{2}
$$

The center of the first pad is found with the phase mirror (PM) set to "max_pm" by detecting and averaging both pad edges parallel to the path progression. Figure (5.15) visually represents an example of this implementation. The location of each pad (BM, FM, and PM) and the corresponding wavelength and power is stored, which is processed when the program ends.

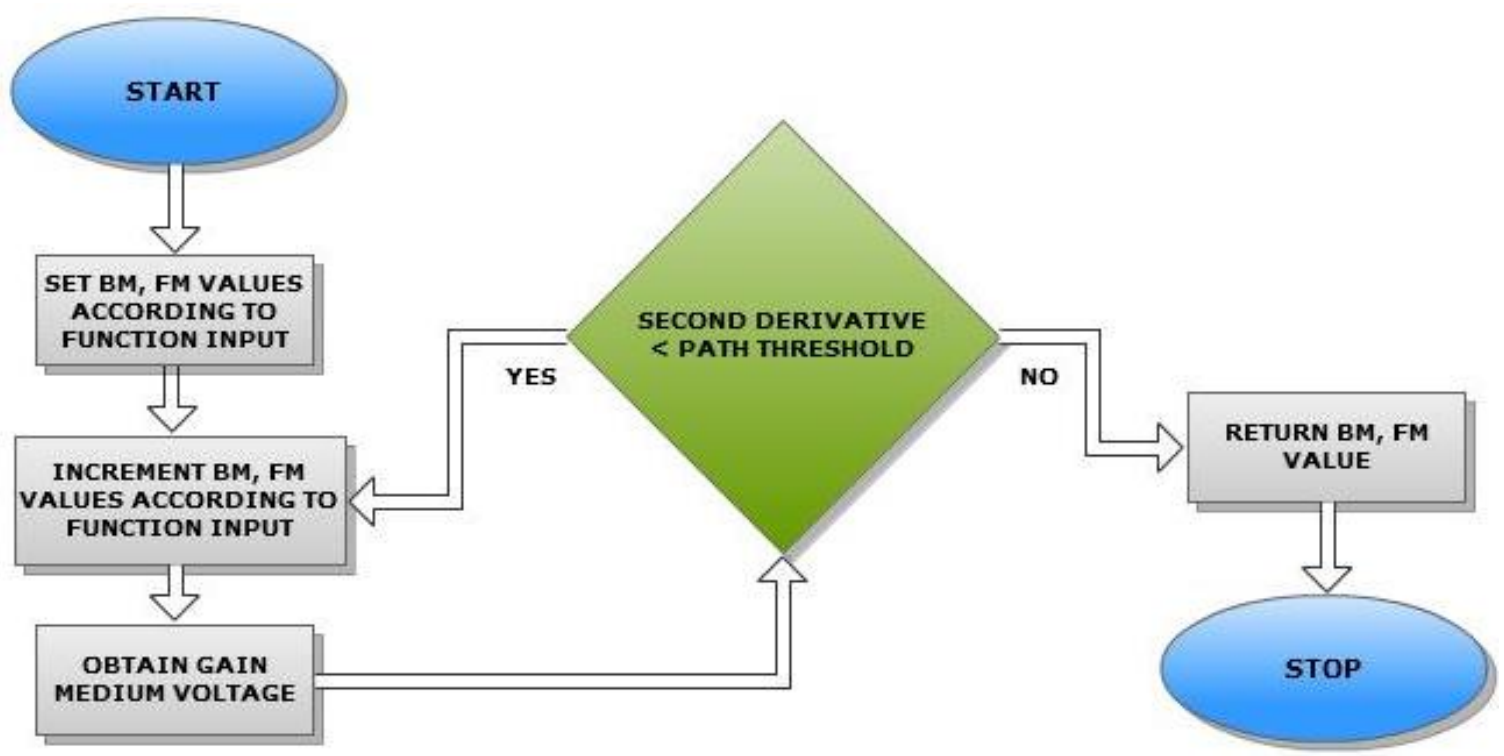

Figure 5.14: Software flowchart for mode change detection 
As seen in figure (5.16), the program begins to traverse the low region of the path. This is accomplished by finding centers while decrementing PM and moving towards the start points within the path. Once either BM or FM reaches "min_bm" or "min_fm" respectively, low region path extension is attempted, which will be explained in detail in subsection (5.3.1). After the possible extension, the BM and FM values are reinitialized to the first center with PM set to "min_pm". Subsequent centers are found while incrementing PM and moving towards the stop points within the path. As before, once either BM or FM reaches “max_bm” or “max_fm” respectively, high region path extension is attempted. Figure (5.17) visually represents the various functions during the traversal of a path.

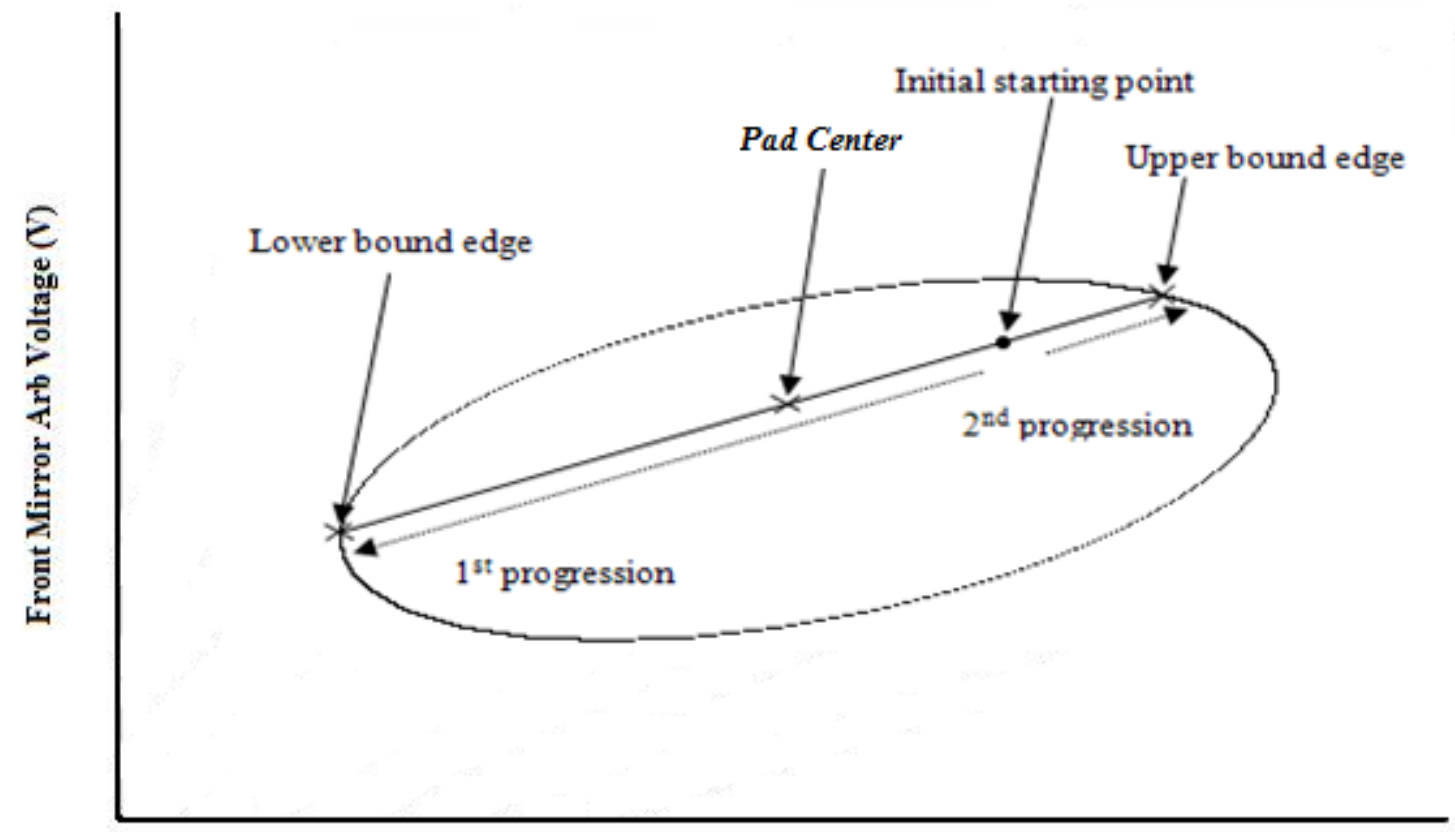

Back Mirror Arb Voltage (V)

Figure 5.15: Visual representation of locating the center of a pad 


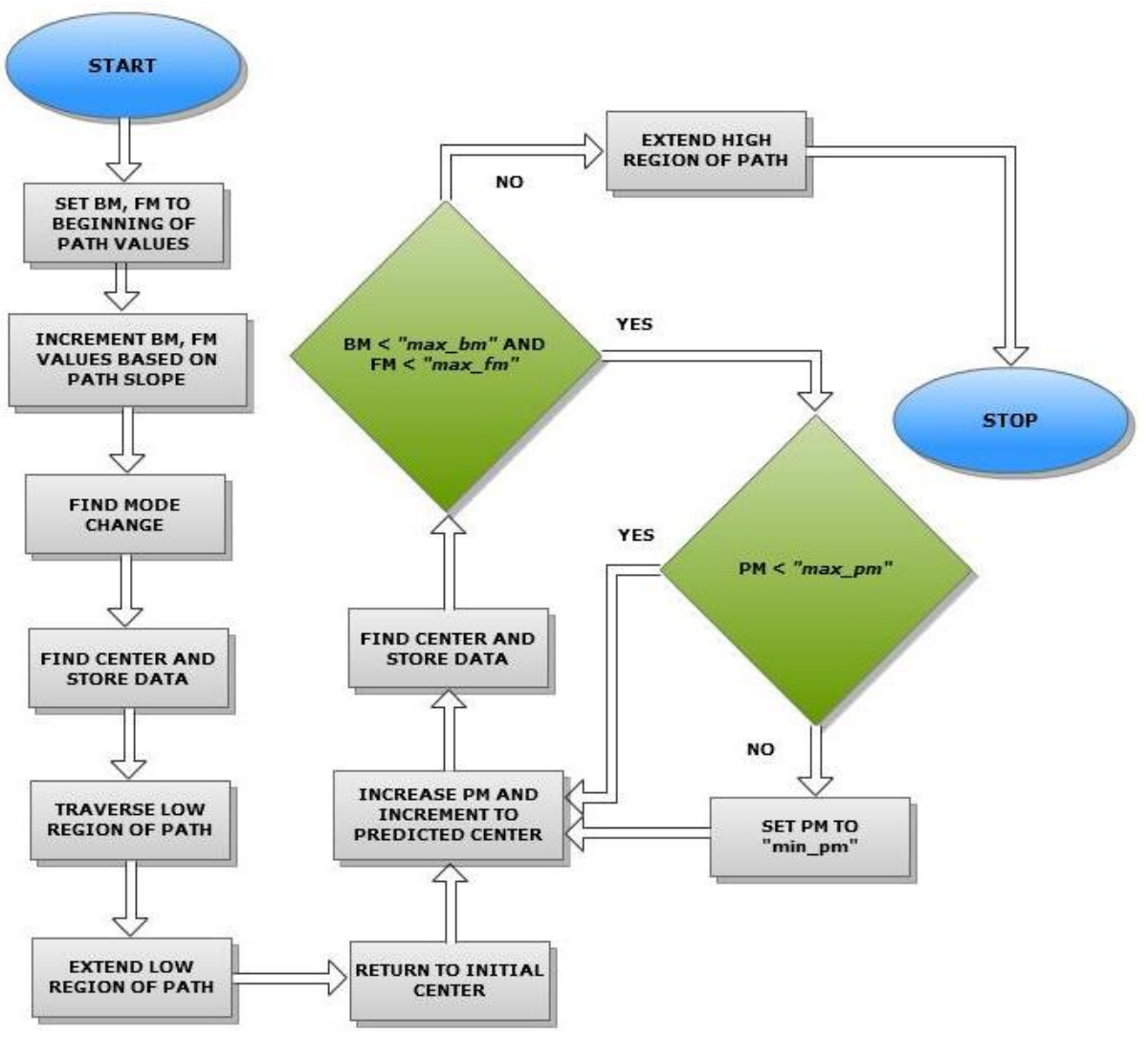

Figure 5.16: Software flowchart for traversing a path

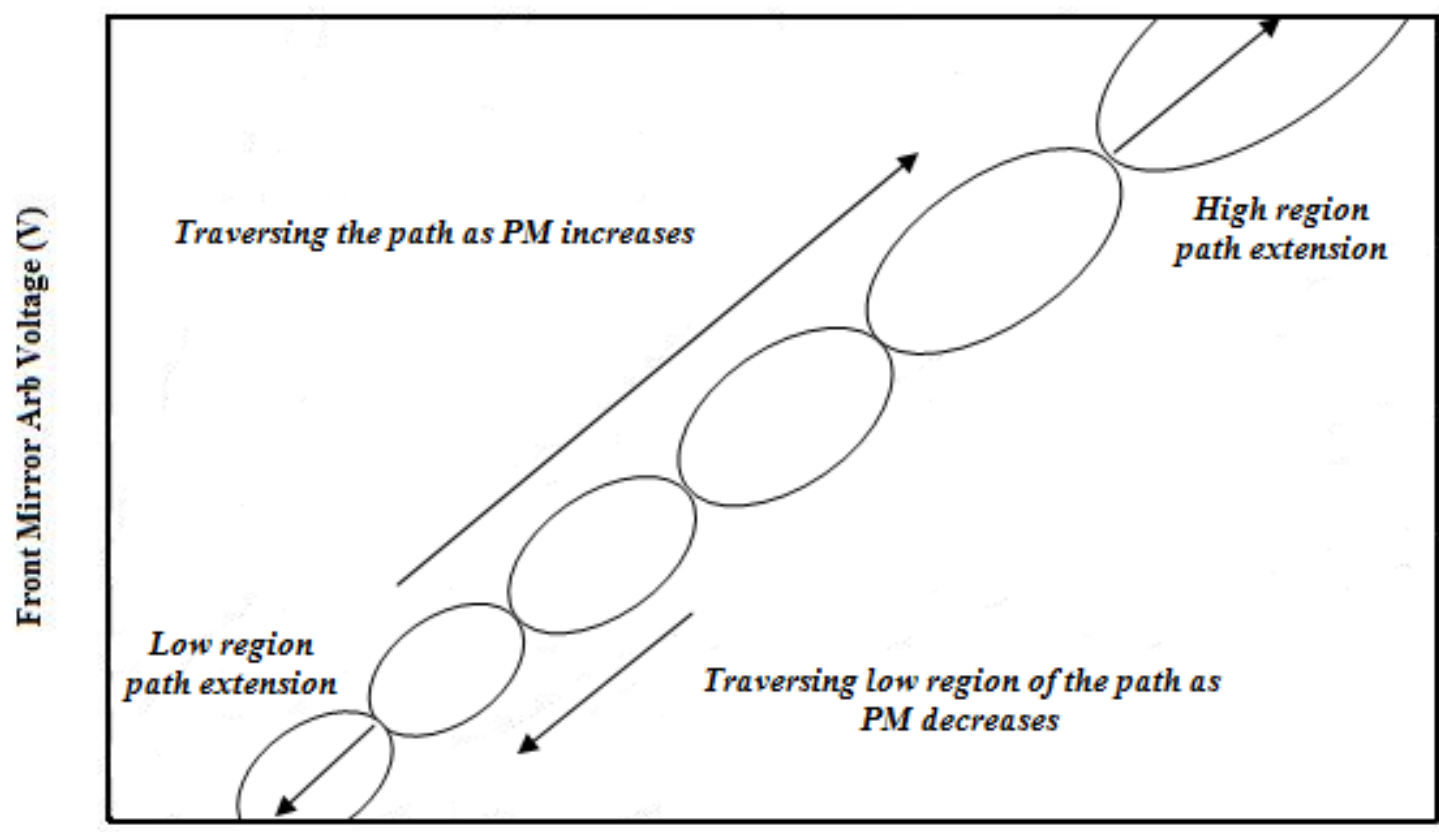

Back Mirror Arb Voltage (V)

Figure 5.17: Visual representation of various functions while traversing a path 


\subsection{1 - Path Extension}

It's important to perform path extension so wavelength overlap exists between paths. However, this poses a challenge of finding the correct centers because two pad edges are needed, but only one exists. Each diode within the laser is limited to a specific maximum current that should not be exceeded. As depicted in figure (5.17), only part of a pad might exist within the confines of the operating region. Therefore, the following algorithm is implemented to project centers that are undetectable with the typical methodology.

Over the progression from the beginning to the end of the path, the pads grow in size. Once the extension begins, the size of the extended pad is projected based on the growth rate from the previous pads. Therefore, the extended center can be predicted based on the measured lower mode change and the projected distance from that mode change. Figure (5.18) visually represents this implementation.

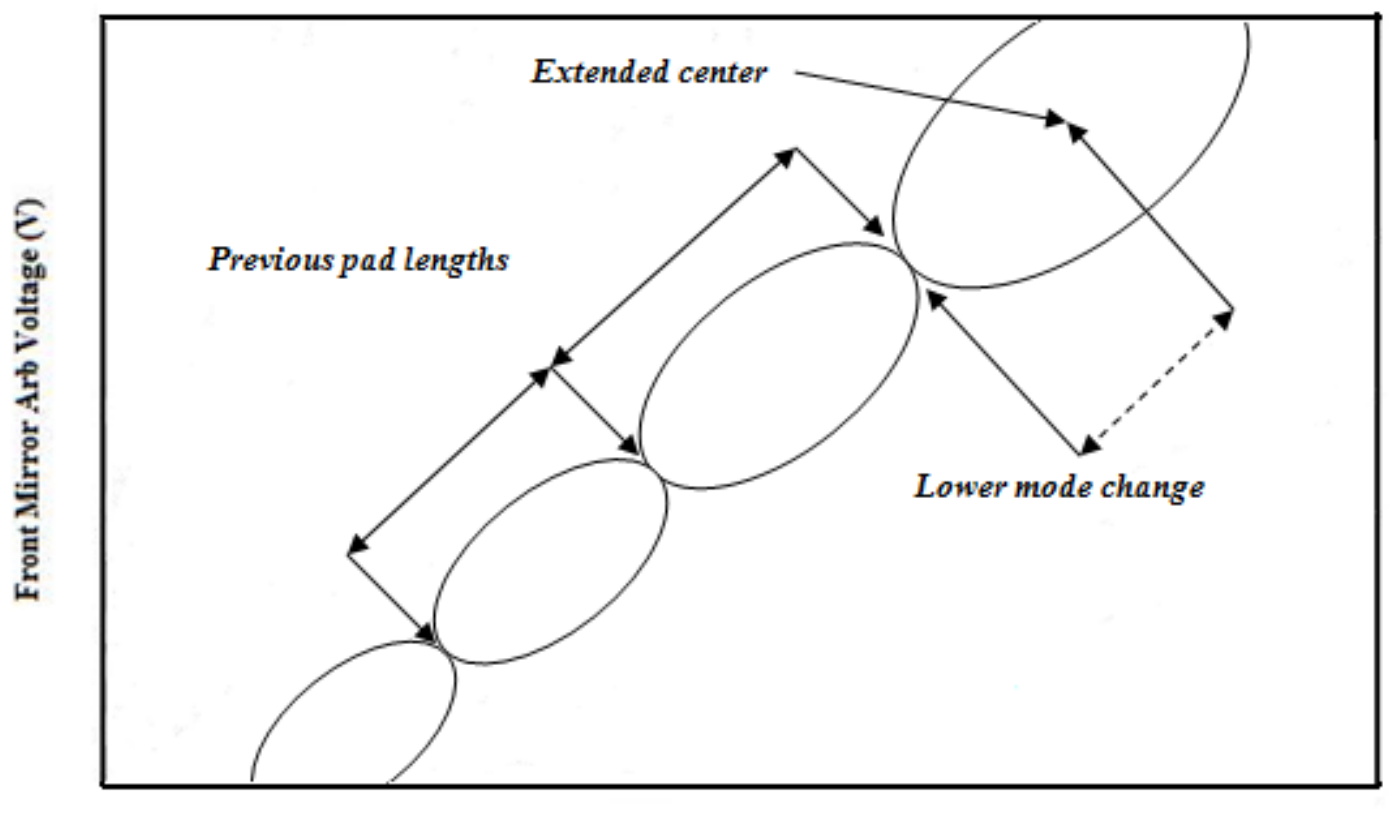

Back Mirror Arb Voltage (V)

Figure 5.18: Visual representation of path extension 


\section{4 - Data Processing}

Once path traversal has completed for every path, data processing begins. This process is threefold: linearly frequency interpolating data, stitching phase sets and paths, and power leveling. Each center is recorded throughout the searching algorithm and stored in a data structure similar to table (5.1). A "phase set" is denoted as a set of center values found within the same progression of PM. Once PM is reset to "min_pm", the phase set is incremented unless it is part of a new path. In that case, "path" is incremented within the data structure. This numbering system simplifies the data stitching and insures that overlap between phase sets and paths exist.

Table 5.1: Example data structure used to process and save data

\begin{tabular}{c|c|c|c|c|c|c|c} 
Path & Phase Set & Pt. & BM (V) & FM (V) & PM (V) & Wavelength (m) & Power (dBm) \\
\hline \hline 0 & 4 & 0 & 3.2780 & 1.3382 & 0.3000 & $1.52426 \mathrm{E}-06$ & 4.7682 \\
0 & 4 & 1 & 3.5509 & 1.4398 & 0.5325 & $1.52399 \mathrm{E}-06$ & 4.5699 \\
0 & 4 & 2 & 3.8312 & 1.5442 & 0.7650 & $1.52374 \mathrm{E}-06$ & 4.3615 \\
0 & 4 & 3 & 4.0426 & 1.6229 & 0.9975 & $1.52357 \mathrm{E}-06$ & 4.1938 \\
0 & 4 & 4 & 4.2075 & 1.6843 & 1.2300 & $1.52344 \mathrm{E}-06$ & 4.0414 \\
1 & 0 & 0 & 0.5432 & 0.3613 & 0.7650 & $1.53547 \mathrm{E}-06$ & 7.2624 \\
1 & 0 & 1 & 0.5691 & 0.3732 & 0.9975 & $1.53533 \mathrm{E}-06$ & 7.1767 \\
1 & 0 & 2 & 0.5945 & 0.3848 & 1.2300 & $1.53521 \mathrm{E}-06$ & 7.0981 \\
1 & 0 & 3 & 0.6152 & 0.3943 & 1.4625 & $1.53511 \mathrm{E}-06$ & 7.0585 \\
1 & 0 & 4 & 0.6328 & 0.4024 & 1.6950 & $1.53503 \mathrm{E}-06$ & 7.0054
\end{tabular}

As previously mentioned in chapter 3 , the approximate 650 center values are linearly $100 \mathrm{MHz}$ frequency interpolated to approximately 52,000 points. This is performed before phase and path stitching, which creates a higher continuity between transition points. After interpolation, stitching, and power leveling, the program produces seven text files: "BM.txt”, "FM.txt”, "PM.txt”, "SOA.txt”, "scaling.txt”, “calibration_data.txt”, and "interp_calibration_data.txt”. The first four listed files contain the interpolated normalized synchronized waveform data where the appropriate 
scaling constants are available in "scaling.txt". The last two files contain preinterpolated and interpolated data with the specific phase set and path number corresponding to center locations. The preinterpolated file is created whether or not a successful calibration completes. This allows the user to understand why an error might have occurred.

Wavelength stitching is extremely important to insure that overlaps exist between adjacent phase sets and paths. This is accomplished by removing significant overlap data. For example, for a path to path stitch, data from the end of path 3 is removed and stitched to the same or slightly greater wavelength in the beginning of path 2 . This same process is also implemented between phase sets. The lower current data is always retained to improve the power consumption within the laser.

\subsection{1 - Power Leveling with the SOA}

A property of the SG-DBR laser is that the output power of the laser is dependent on the input currents that tune the laser. A frequency ramp with level output power versus frequency is needed for various types of optical sensing techniques, including OCT [15]. As previously discussed, the SOA current controls the output power and therefore needs to be included in the calibration. A full characterization at one wavelength of the SOA seen in figure (5.19) was obtained before the power leveling is implemented. This is necessary because the SOA has a nonlinear input current vs. output power relationship as well as a nonlinear relationship with respect to frequency. The graph depicted is taken by sweeping the SOA from 2 to 5 volts (66 mA to $166 \mathrm{~mA})$ at the center wavelength of the laser. The desired power level for calibrated lasers is $10 \mathrm{dBm}$ where the trend line equation shown provides an SOA value for each data point based on the previous power difference. 


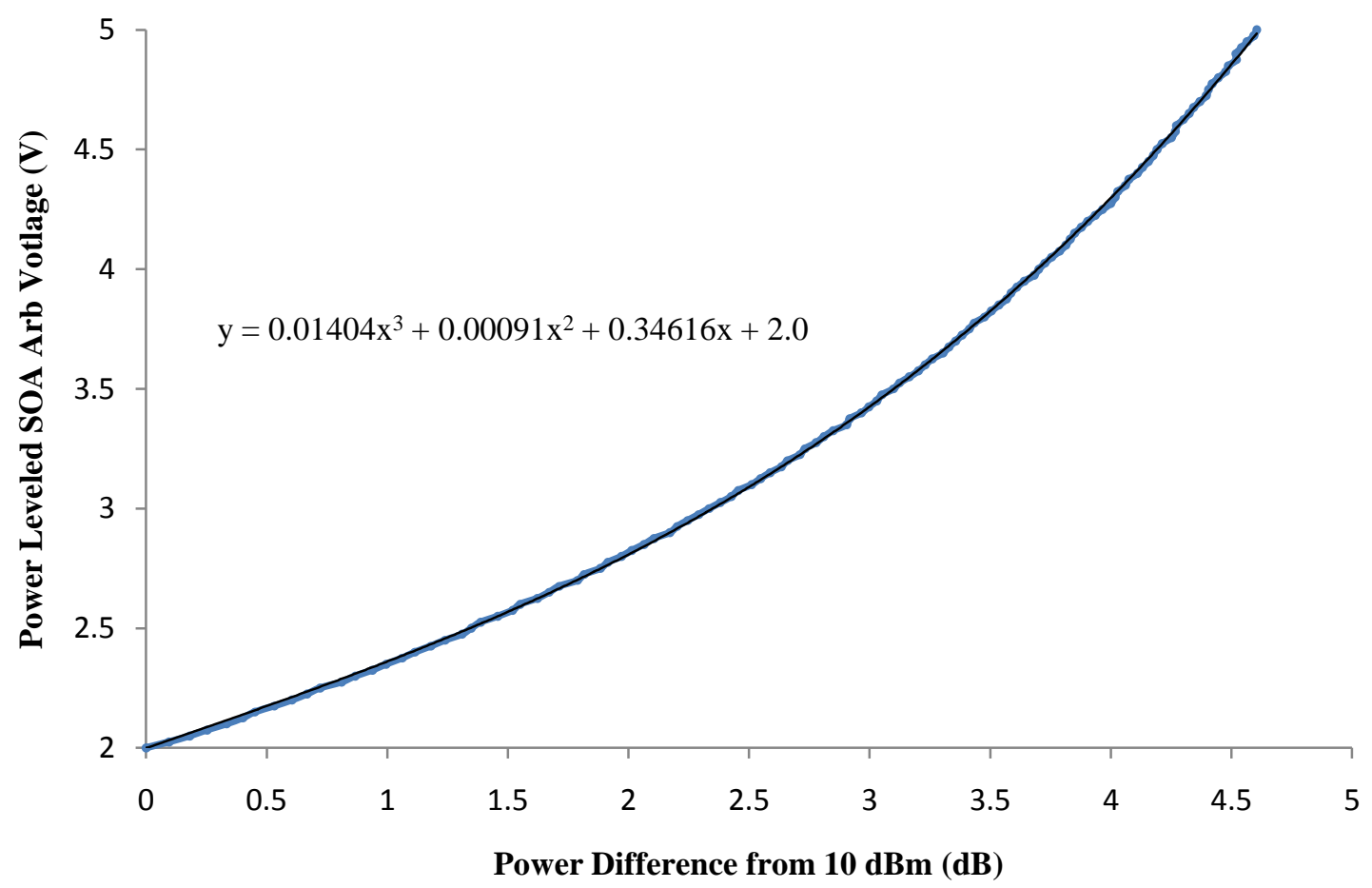

Figure 5.19: Graph depicting the relationship between the gain needed to adjust the power level to 10 $\mathrm{dBm}$ and the corresponding SOA value 


\section{5 - Output Data and Results}

Once the full linear power leveled frequency ramp is assembled, the BM, FM, PM, and SOA sets of data (DATA (DATA $_{\text {NORMALIZED }}$ ) between 0 and 1 with their corresponding scale factors $(\mathrm{SF})$. This is done according to equations (5.2-5.3). This is necessary because the Agilent Waveform editor software which allows the user to input arbitrary waveform data to the function generators requires this range at initialization. Figures (5.20-5.23) show the normalized arbitrary waveforms for the C-Band laser that the automated calibration program generated and figure (5.24) shows the corresponding linear frequency ramp.

$$
\begin{gathered}
\text { DAT } A_{\text {NORMALIZED }}=\frac{D A T A_{\text {UNNORMALIZED }}}{5} \\
S F=5\left(M A X\left(D A T A_{\text {NORMALIZED }}\right)-\operatorname{MIN}\left(D A T A_{\text {NORMALIZED }}\right)\right)
\end{gathered}
$$

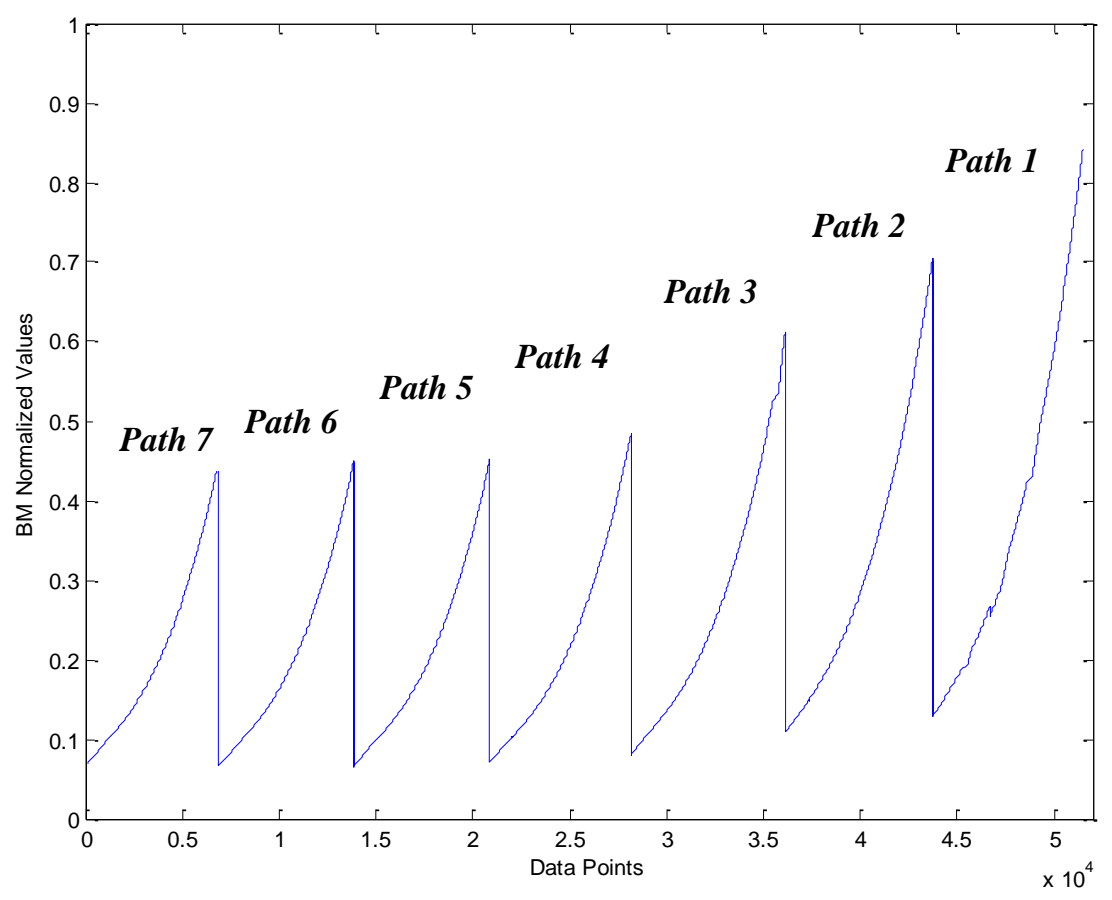

Figure 5.20: Input normalized BM arbitrary waveform data used to control the linear frequency ramp for the $\mathrm{C}$-Band laser 


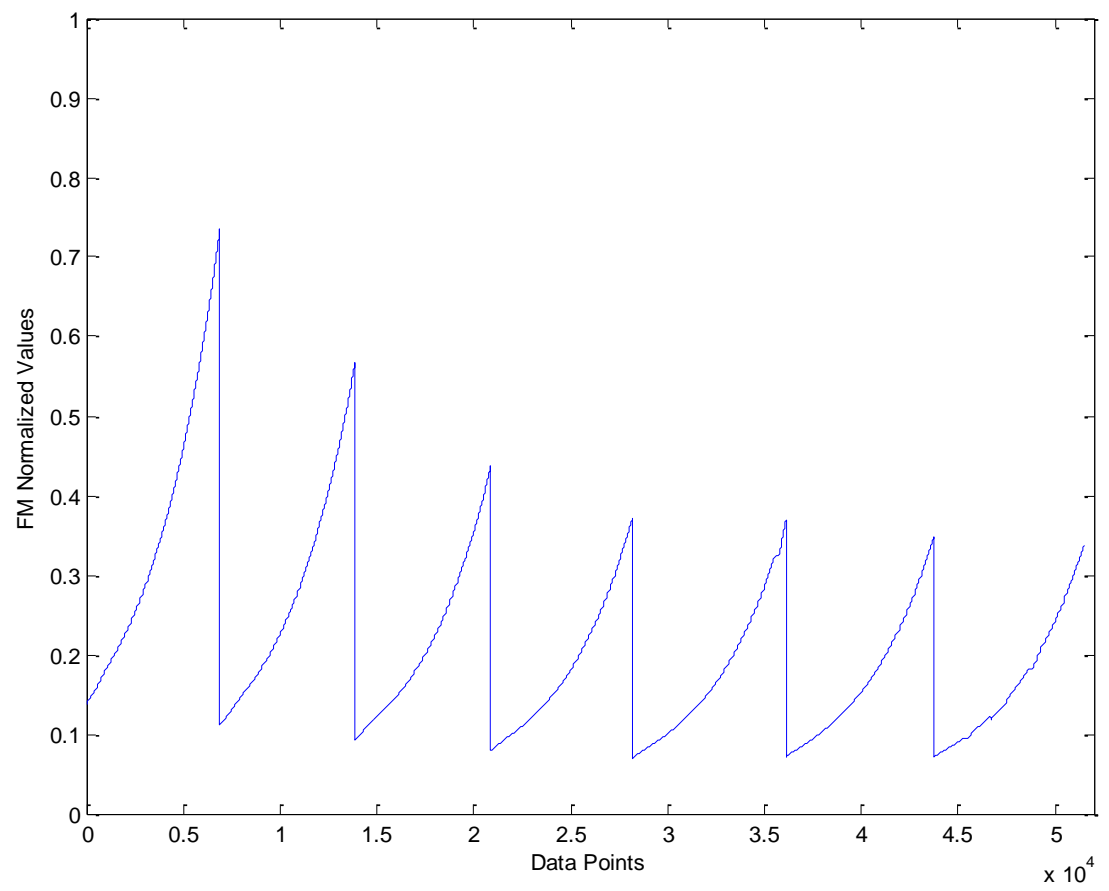

Figure 5.21: Input normalized FM arbitrary waveform data used to control the linear frequency ramp for the $\mathrm{C}$-Band laser

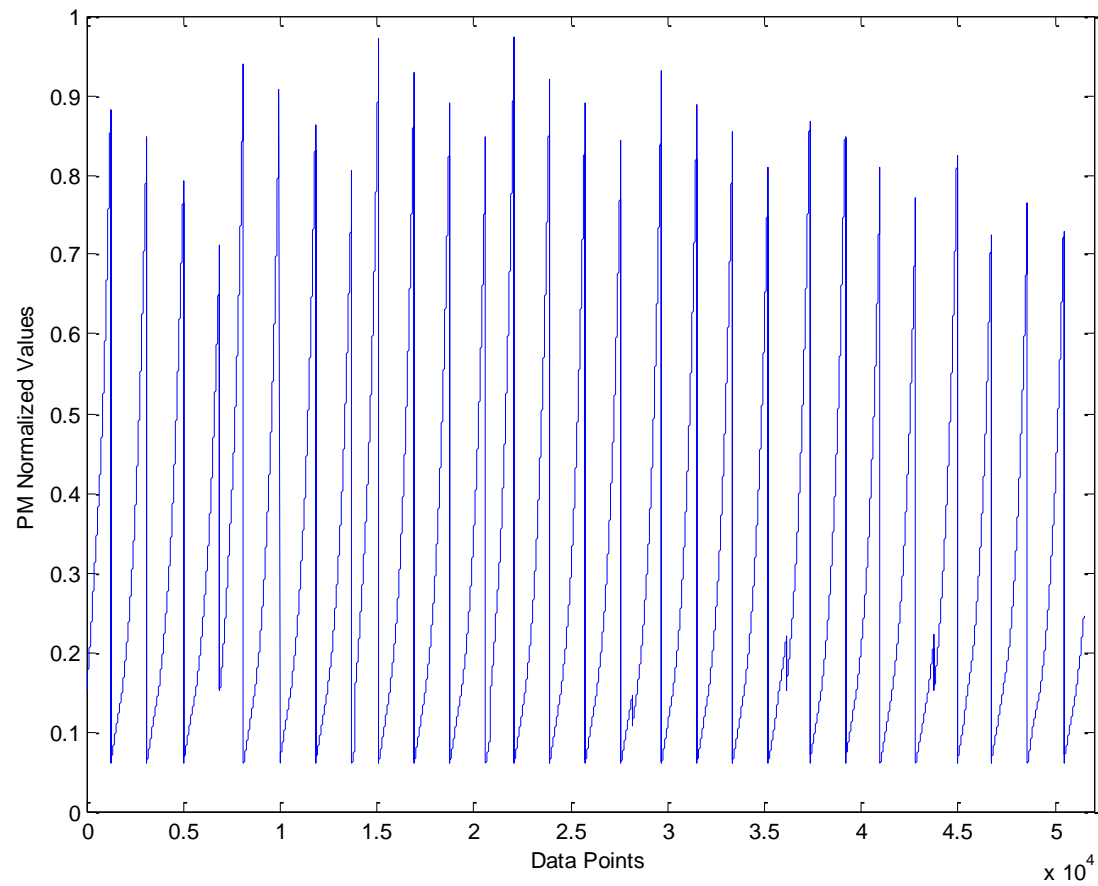

Figure 5.22: Input normalized PM arbitrary waveform data used to control the linear frequency ramp for the C-Band laser 


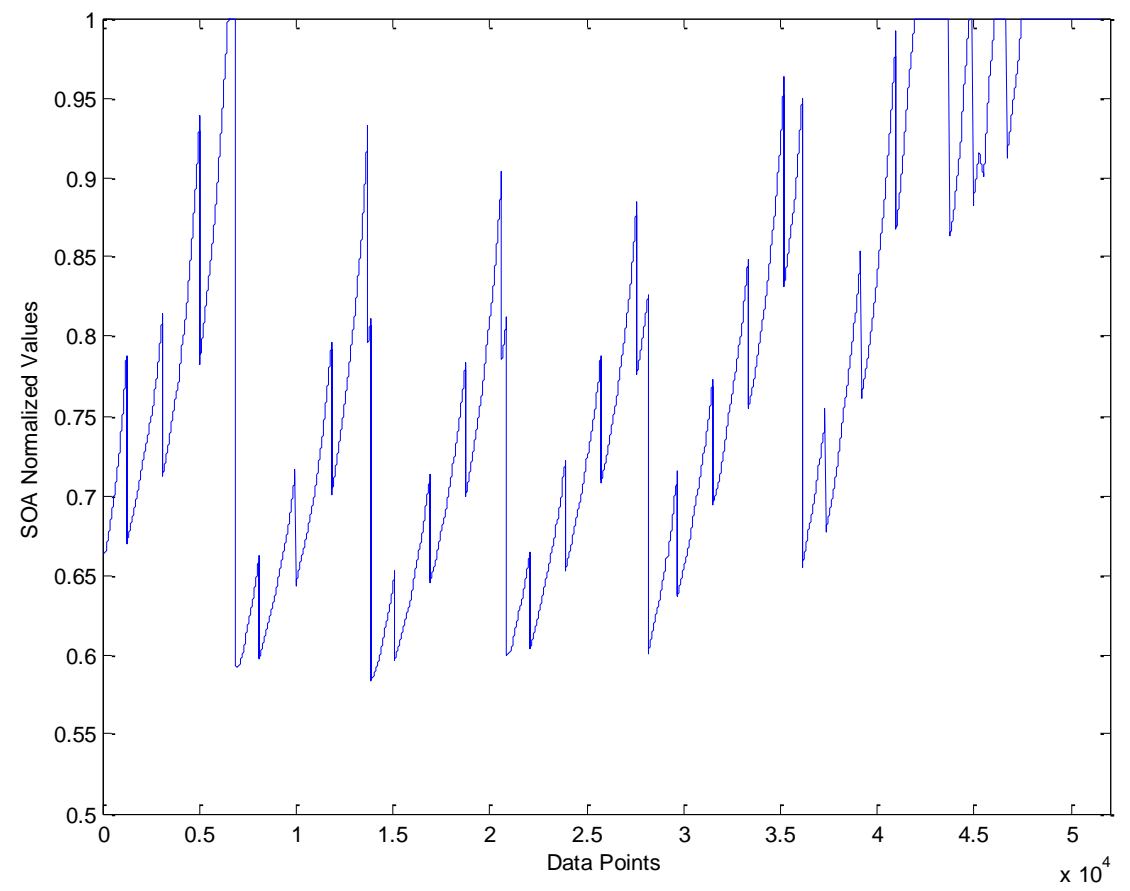

Figure 5.23: Input normalized SOA arbitrary waveform data used to control the linear frequency ramp for the C-Band laser

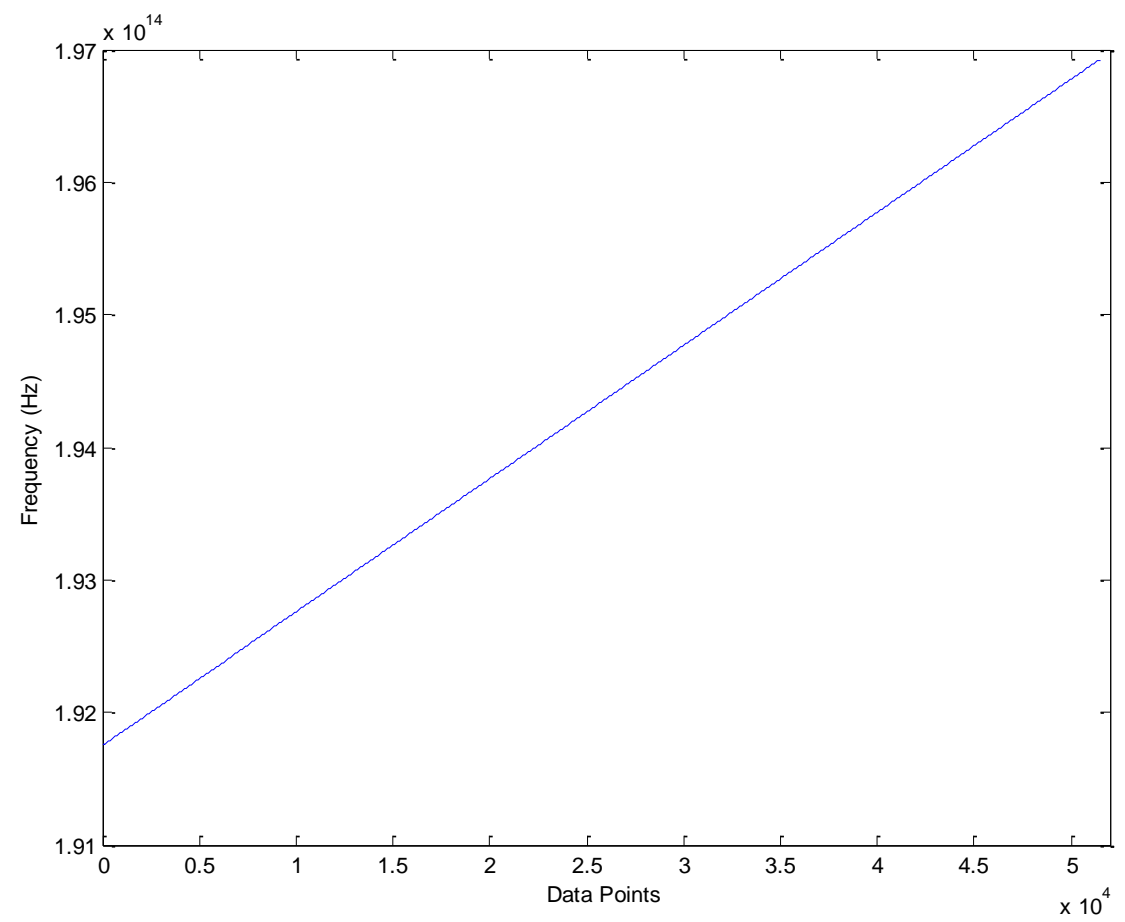

Figure 5.24: Corresponding linear frequency ramp 
To implement the four waveforms, the waveform editor program requires two inputs before sending the files to the function generators. Each scale factor is needed and a repetition rate. For the initial assessment of results, a $1 \mathrm{kHz}$ repetition rate was chosen which means that all four sets of approximately 52,000 points are continuously swept over the duration of 1 millisecond. The waveforms are synchronized with an external trigger set to $900 \mathrm{~Hz}$. Figure (5.25) shows the output linear frequency ramp from the OSA which validates a fully functional automated calibration program. Table (5.2) provides a summary of the automated sweeping C-Band performance compared to the previous manual calibration.

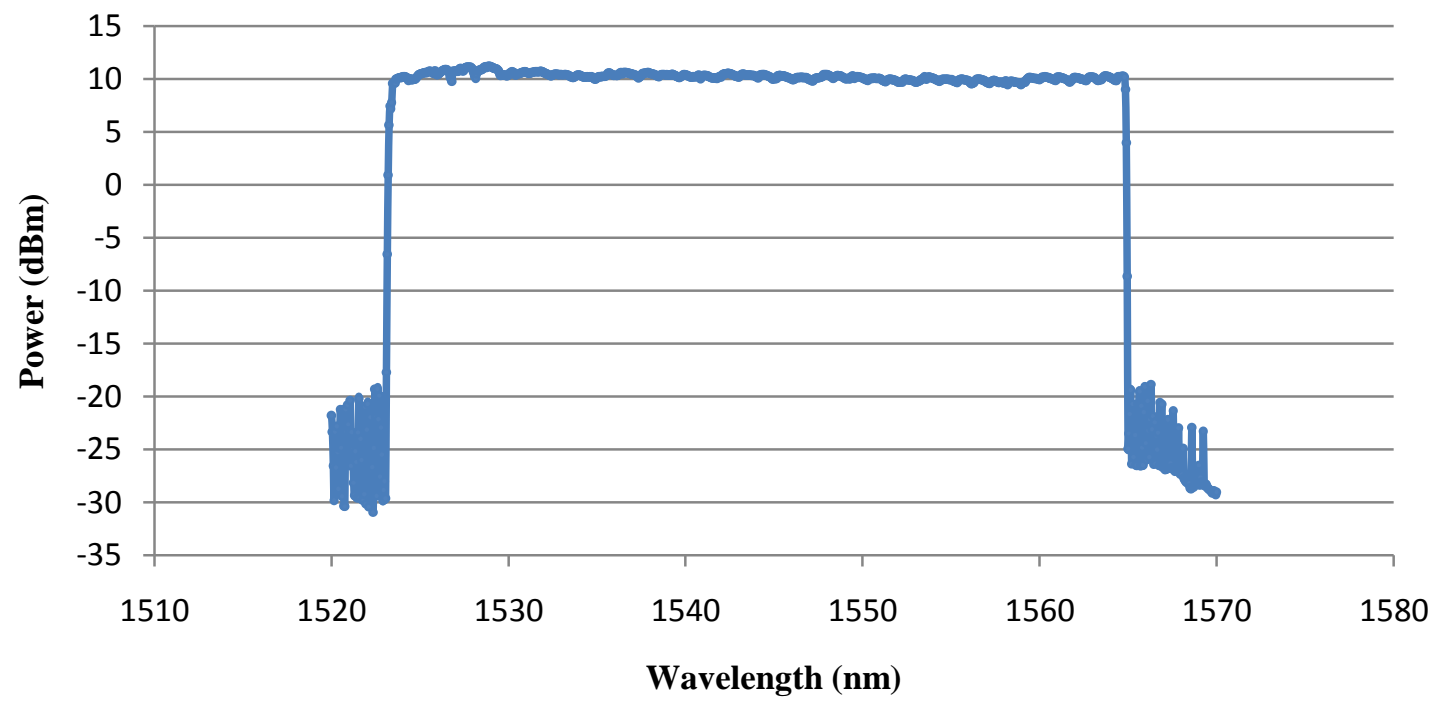

Figure 5.25: Output frequency ramp from the OSA with a $1 \mathrm{kHz}$ repetition rate

Table 5.2: Summary of C-Band performance compared to the manual calibration

\begin{tabular}{c|c|c} 
& Manual C-Band & Automated C-Band \\
\hline \hline Average Power & $11.282 \mathrm{dBm}$ & $10.205 \mathrm{dBm}$ \\
Std. Deviation of Power & $0.3747 \mathrm{dBm}$ & $0.3277 \mathrm{dBm}$ \\
Bandwidth & $41.2 \mathrm{~nm}$ & $41.35 \mathrm{~nm}$ \\
Calibration Time & $\approx 48$ hours & $\approx 10$ hours
\end{tabular}




\section{6 - Assessment of Output Linearity}

To assess the output linearity, the sweeping laser output is connected into a 7.1 GHz free spectral range (FSR) Mach-Zehnder interferometer which produces a sinusoidal interference pattern. This implementation allows for a thorough analysis of the spacing of zero-crossings within the interference pattern and the effects of the rapid waveform switching which cause stitching imperfections.

\subsection{1 - Statistical Analysis of Zero-Crossings of Interference Pattern}

Detecting zero-crossings of the sinusoidal interference pattern provides an assessment of how reliable a distance is measured. Figure (5.26) shows one section of the interference pattern where one period is related to a $7.1 \mathrm{GHz}$ frequency difference in the linear frequency ramp.

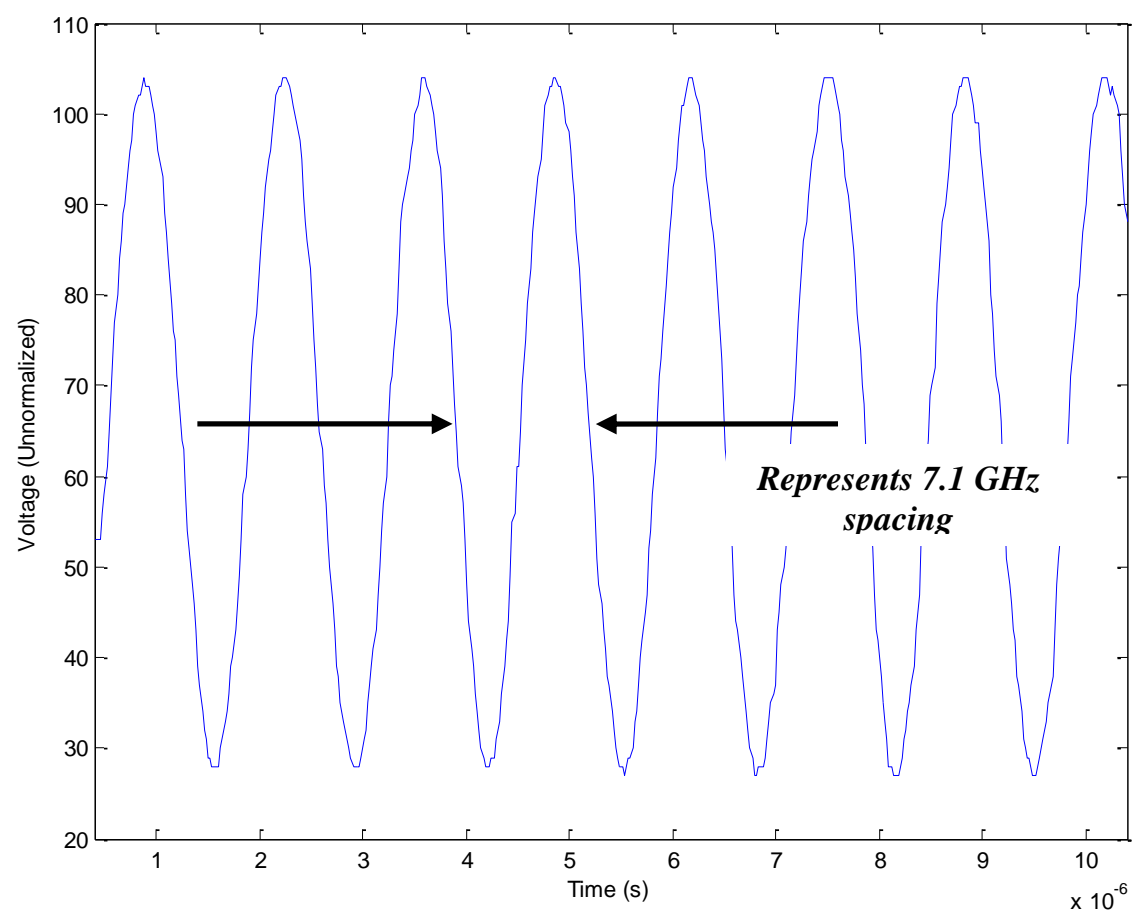

Figure 5.26: Interference pattern produced by a 7.1 GHz FSR interferometer at a $1 \mathrm{kHz}$ sweep rate 
The Mach-Zehnder interferometer emulates a measured distance that is ideally constant. With a perfect linearly frequency sweeping laser, the time between zerocrossings should be the same throughout the duration of the sweep. To assess this linearity, a MATLAB script was written to detect zero-crossings, determine the time of these intervals, and produce the histogram displayed in figure (5.27). Any significant frequency modulation throughout the sweep will essentially produce false distance information. Therefore, a low standard deviation of zero-crossing time intervals is desired and provides evidence of sweep linearity. There was slight frequency modulation throughout the sweep. On average, the frequency range can be calculated to be $193.6 \mathrm{kHz}$ by equation (5.3), ranging from $697.5 \mathrm{kHz}$ to $891.1 \mathrm{kHz}$.

$$
\Delta f=\frac{1}{2\left(\bar{x}-\sigma_{x}\right)}-\frac{1}{2\left(\bar{x}+\sigma_{x}\right)}
$$

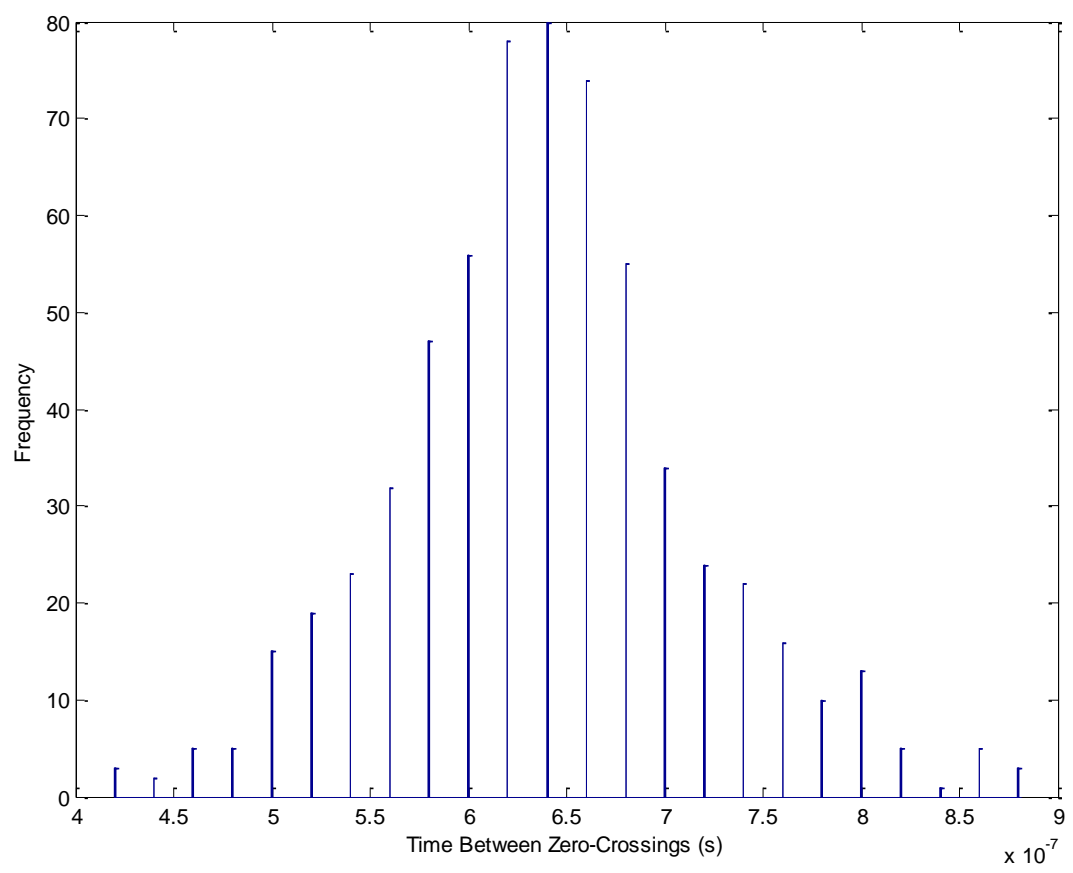

Figure 5.27: Histogram of time between zero-crossings with a mean of 639 ns and standard deviation of $77.89 \mathrm{~ns}$ 


\subsection{2 - Assessment of Stitching Imperfections}

Stitching imperfections occur within the interference pattern because of the fastswitching waveforms controlling the laser. Each time when BM, FM, or PM reset to their respective minimum values the function generators are incapable of switching fast enough. An example of this stitching imperfection is seen in figure (5.28). They either cause an underlap or overlap in frequency stitching in which the latter is desired. To assess the stitching imperfections, this qualitative designation is determined for each occurrence which is presented in table (5.3). A method to reduce stitching imperfection effects is detailed in chapter 6 .

Table 5.3: Qualitative determination of stitching imperfections

\begin{tabular}{c|c} 
Qualitative designation & Number of Stitching Imperfections \\
\hline \hline Underlap & 10 \\
Overlap & 25
\end{tabular}

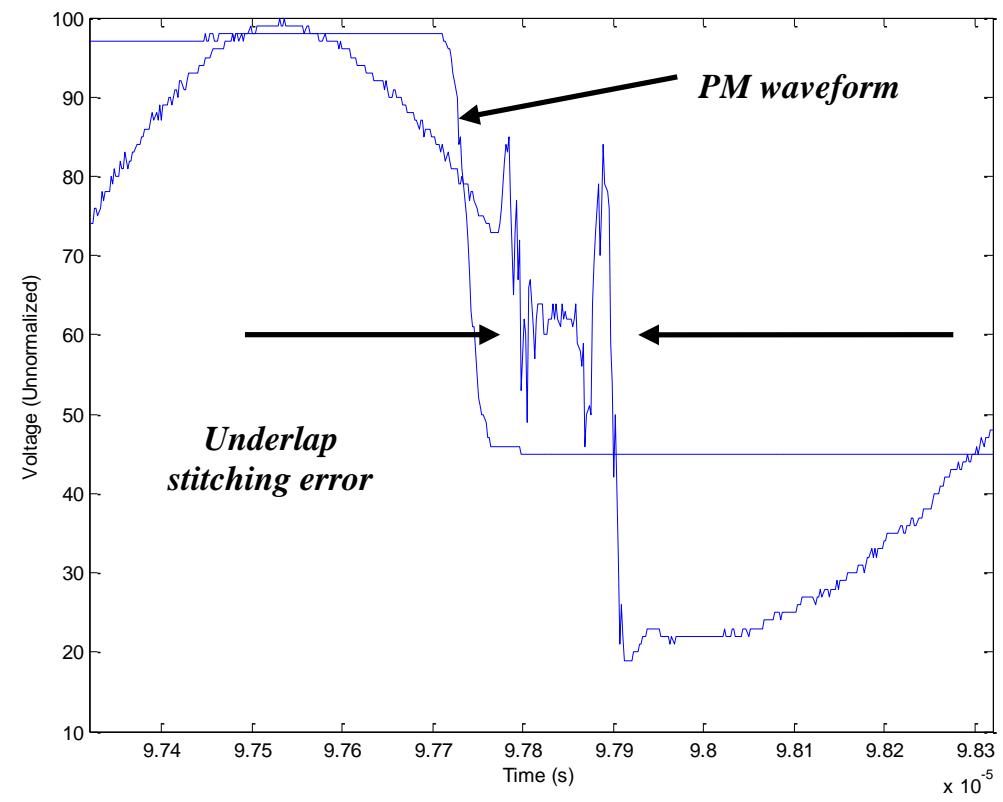

Figure 5.28: Underlap stitching imperfection caused by fast-switching waveform at a $1 \mathrm{kHz}$ sweep rate 


\section{CHAPTER 6 - Swept Source OCT Experimental Results}

It was seen in the previous chapter that the result of fast-switching waveforms cause stitching errors within the interference pattern produced by the tunable laser source. A method to reduce these errors is implemented by utilizing the output of the sweeping laser to ultimately generate an electronic sampling clock. This chapter explores this methodology, provides a brief analysis of how the spectral response improves with the removal of stitching imperfections and how spectral broadening occurs when repetition rate increases.

\section{1 - Instrumentation Setup}

To create the electronic clock, the output of the tunable SG-DBR laser source is first emitted into a 7.1 GHz FSR Mach-Zehnder interferometer. This optical interference pattern is converted to an electrical one with a photodetector and then sampled by an A/D converter. A LabVIEW program detects zero-crossings and produces a square wave at each occurrence. If a stitching error occurs at a zero-crossing, it is manually removed in software. The program then creates an input file of normalized square wave pulses that is sent to a function generator.

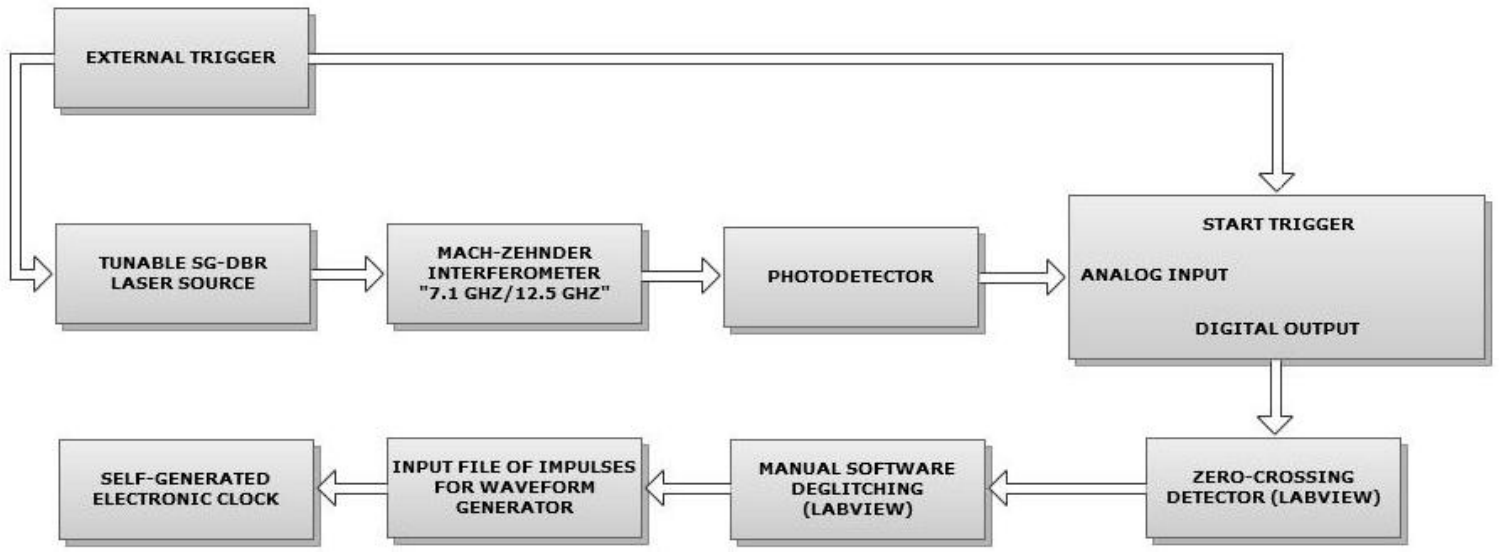

Figure 6.1: Block diagram of instrumentation setup for self-generation of clock 
Once the electronic clock has been generated, it is controlled by the same external trigger controlling the sweeping laser. The interference pattern of a $39 \mathrm{GHz}$ MachZehnder interferometer serving as the DUT is sampled based on the square wave generated from the electronic clock. Figure (6.2) shows a block diagram of this implementation. It's important to note that the interference pattern frequency that's used to generate the sampling is at least two times higher than the interference pattern produced by the DUT which insures that the spectral integrity isn't affected after sampling as depicted in figure (6.3).

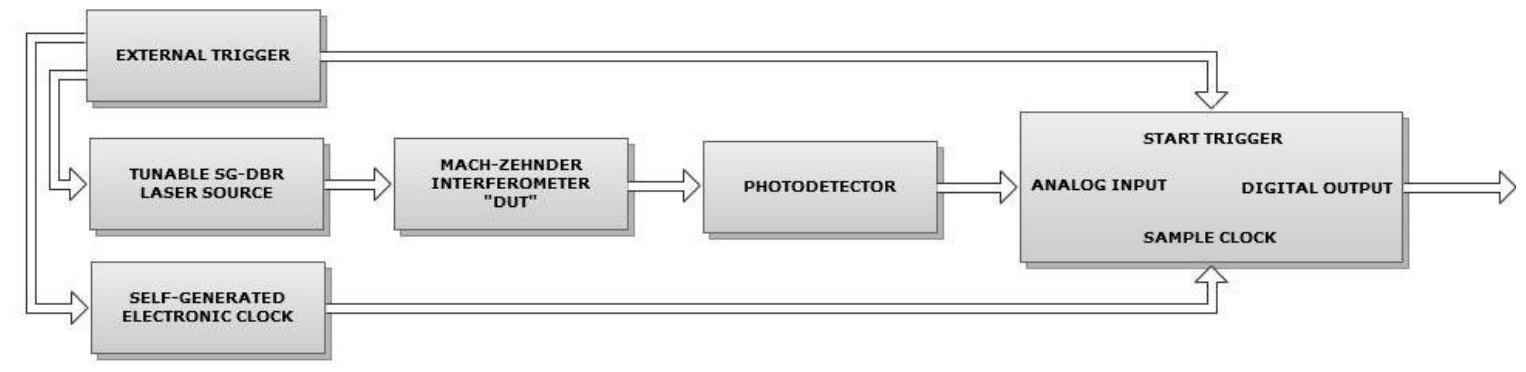

Figure 6.2: Block diagram of instrumentation setup for DUT sampling

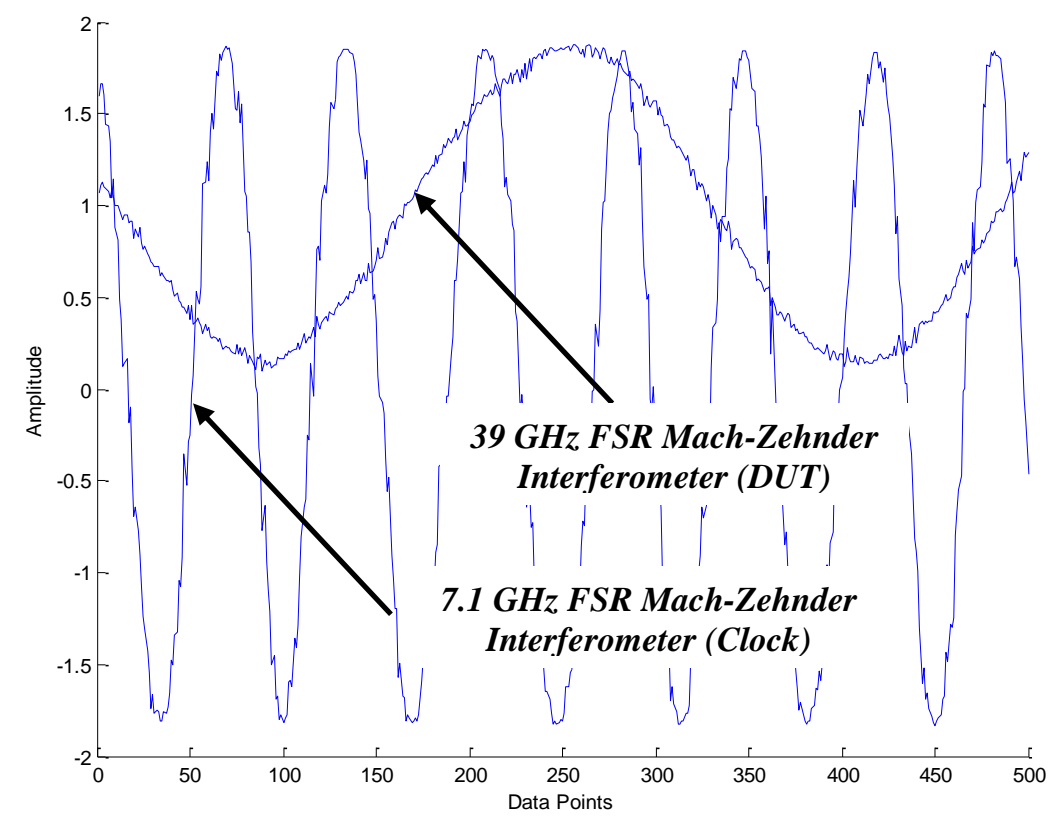

Figure 6.3: Graph of interference patterns for DUT and optical sampling clock 


\section{2 - Improved Spectral Response with Removal of Stitching Errors}

The removal of stitching errors in the clock signal is crucial to improving the spectral response. Irregularities in the initially generated square wave are manually removed so pulses only occur at true zero-crossings. Without removing the errors, samples might be taken at the stitching errors within the DUT signal, which can ultimately cause ghosting effects in the resultant image. Figure (6.4) demonstrates two false square wave pulses generated by stitching errors during zero-crossings. By removing these data points, it reduces the likelihood of sampling false DUT data. This not only causes ghosting effects, but also reduces the one-dimensional distance resolution. Figures (6.5-6.6) show the improvement in the FFT response of the sampled DUT with the removal of clock stitching errors.

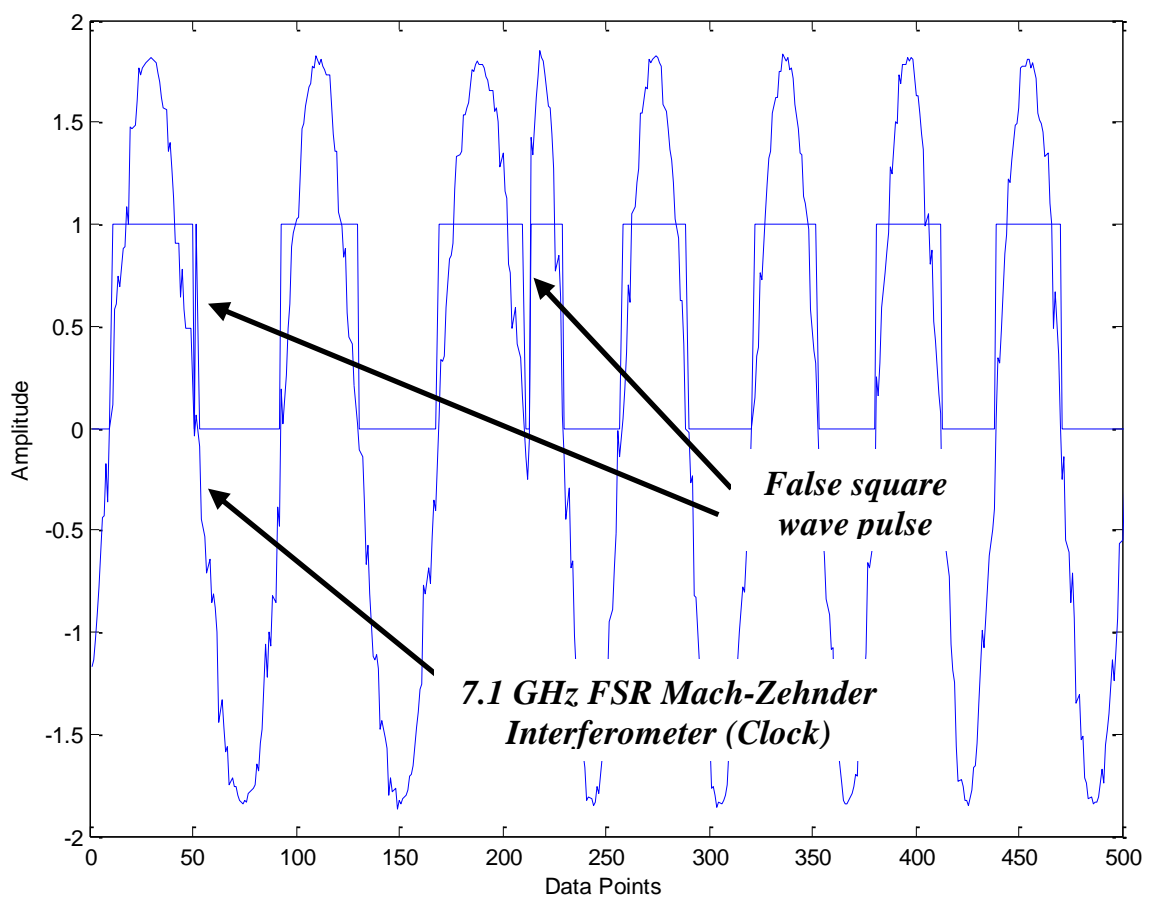

Figure 6.4: Two false square wave pulses generated by stitching errors 


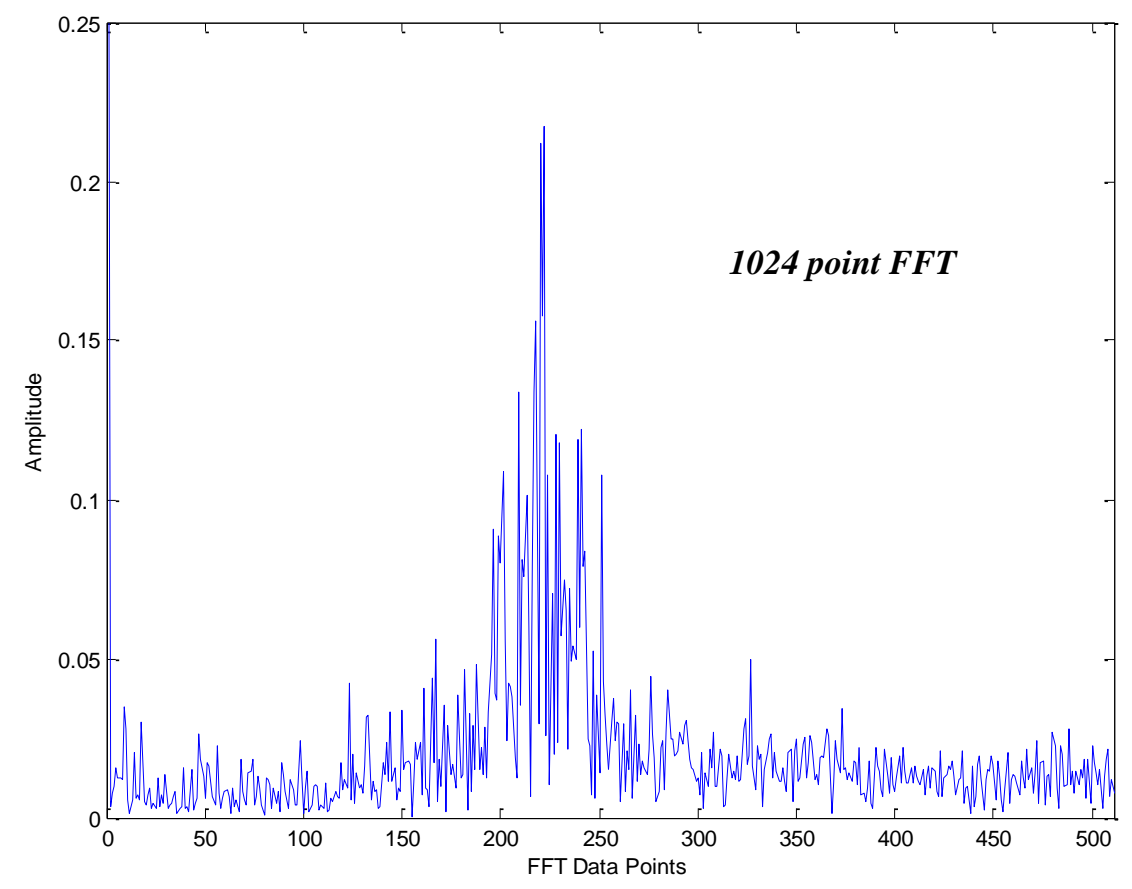

Figure 6.5: FFT response of $39 \mathrm{GHz}$ DUT with no stitching errors removed in clock

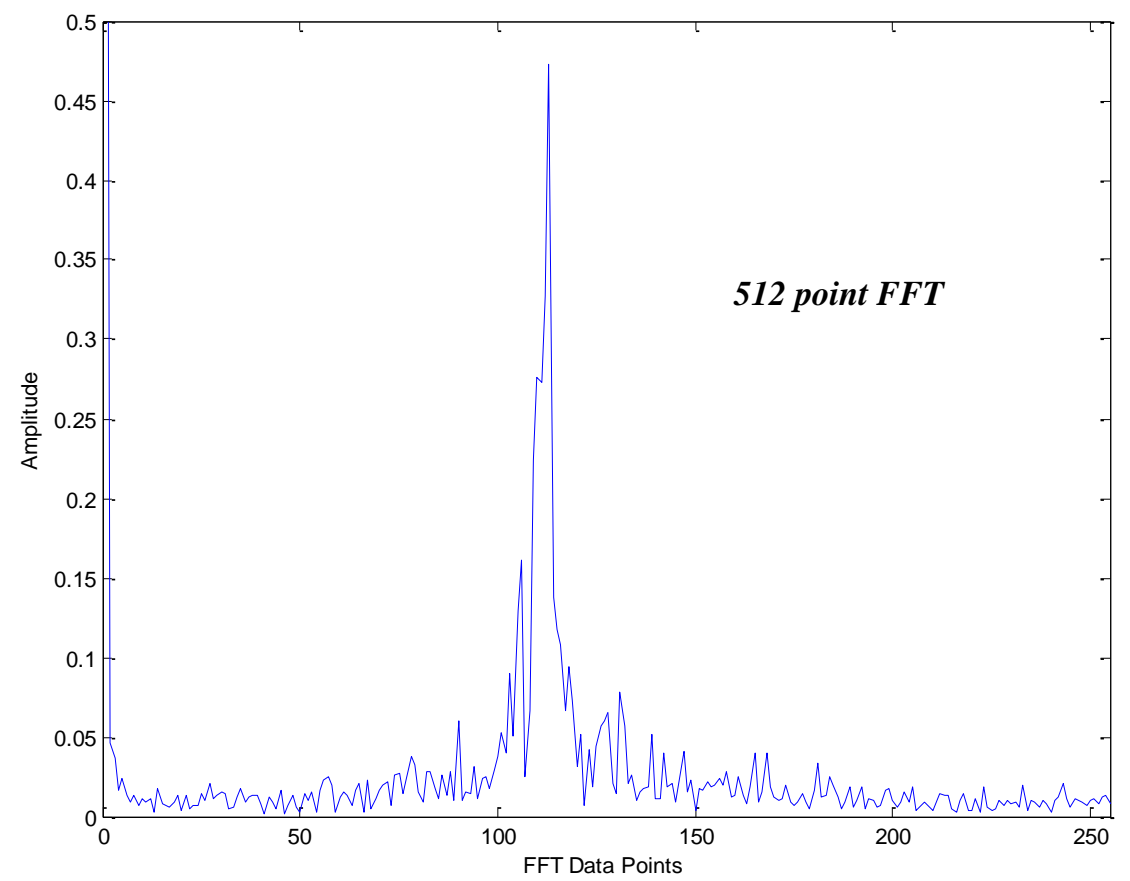

Figure 6.6: FFT response of $39 \mathrm{GHz}$ DUT with all stitching errors removed in clock 


\section{3 - Spectral Broadening as a Function of Sweep Rate}

Spectral broadening is also dependent on the sweep rate of the tunable laser source. This occurs because as the sweep rate increases, the stitching errors become more pronounced. Even with the removal of errors in the clock signal, sampling false DUT data becomes more likely and thus reduces the spectral integrity of the sinusoid as depicted in figures (6.7-6.8). It's important to note that the x-scales for the graphs are adjusted to represent a normalized measured distance that the $39 \mathrm{GHz}$ interferometer emulates. This scaling representation and the summary of system capabilities are explained in further depth in section (6.4).

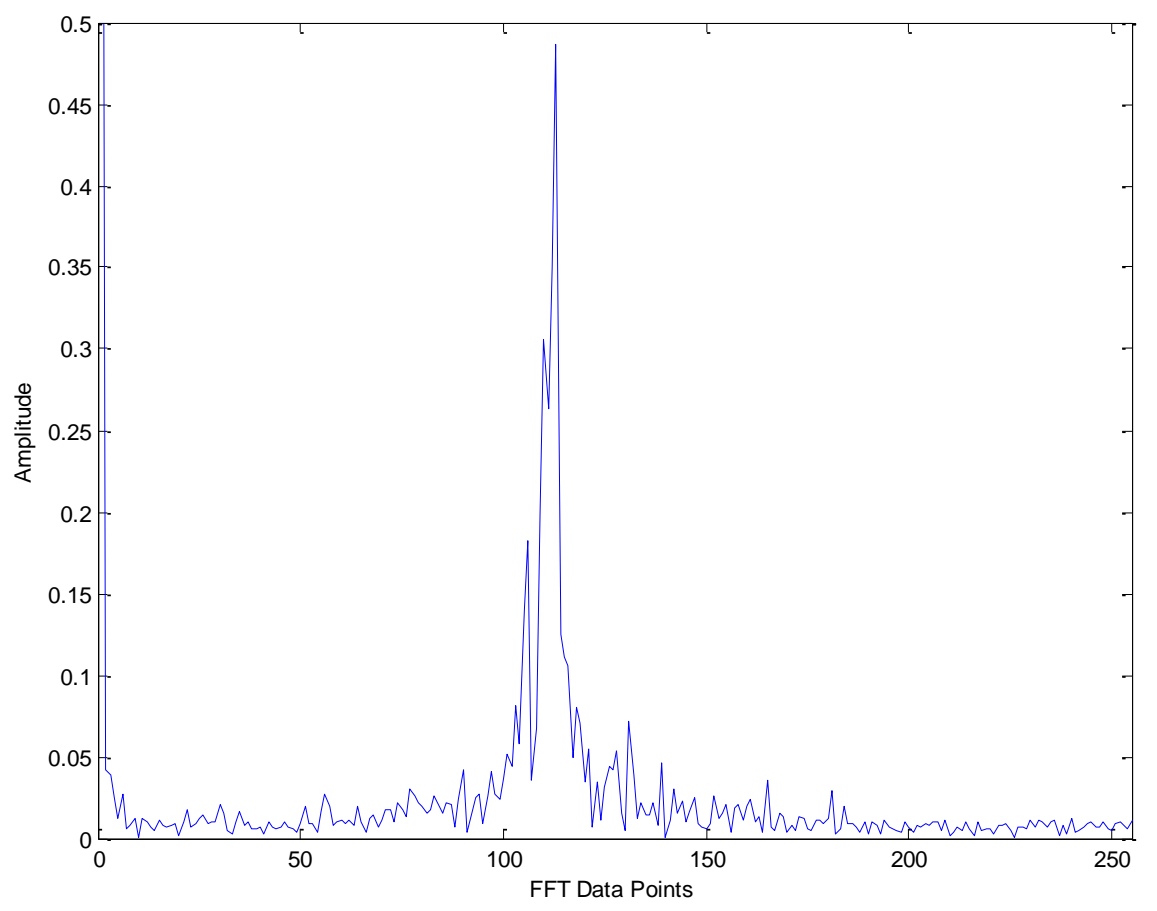

Figure 6.7: FFT response of $39 \mathrm{GHz}$ DUT with a $200 \mathrm{~Hz}$ repetition rate 


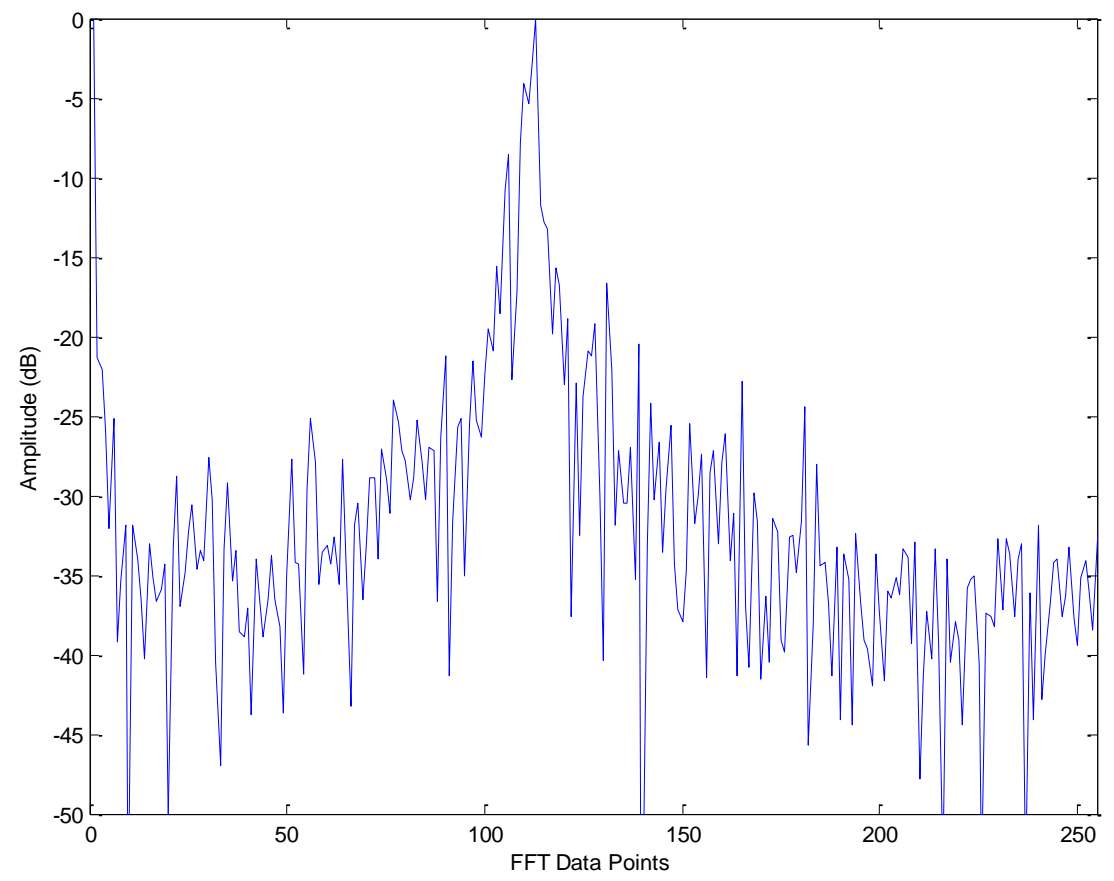

Figure 6.8: FFT response of $39 \mathrm{GHz}$ DUT with a $200 \mathrm{~Hz}$ repetition rate in dB

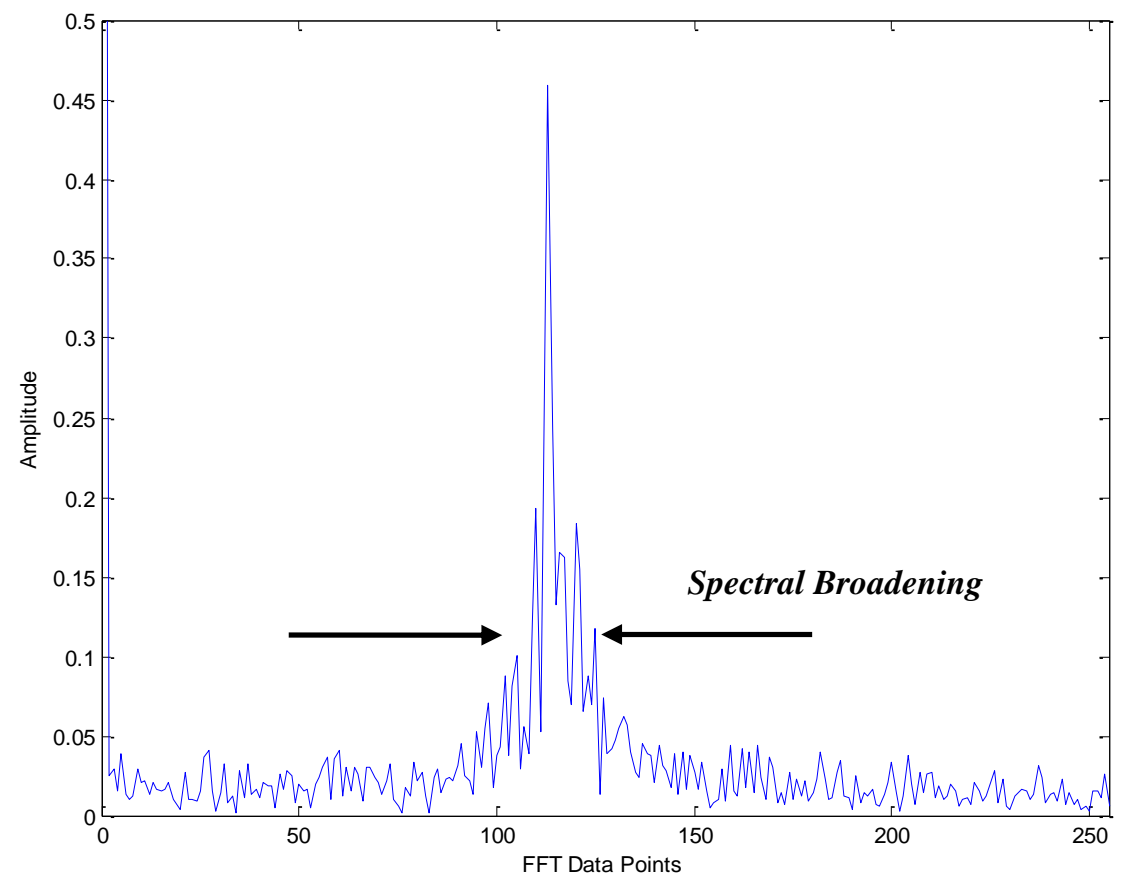

Figure 6.9: FFT response of $39 \mathrm{GHz}$ DUT with a $3 \mathrm{kHz}$ repetition rate 


\section{4 - Summary of Capabilities}

As seen in section (6.2), the spectral response for a $39 \mathrm{GHz}$ FSR Mach-Zehnder interferometer drastically improves with the removal of stitching errors in the selfgenerated clock and with decreased repetition rates. After the DUT signal is sampled based on the electronic sampling clock, an FFT converts the signals to the frequency domain. However, rather than providing a frequency reference, the FFT data points are displayed which can easily be converted to a distance measurement. The sampling rate of the DUT is based on the interference frequency for the $7.1 \mathrm{GHz}$ interferometer. Therefore, according to equations (6.1-6.2) the Nyquist frequency can be calculated where $f_{r}$ is the repetition rate in Hertz, $B W$ is the bandwidth in nanometers, $\Delta \lambda_{\text {int }}$ is the interferometer wavelength difference, $\Delta f_{\text {int }}$ is the interferometer frequency difference, $\lambda_{c}$ is the C-Band center wavelength, and $c$ is the speed of light. These equations show that as the repetition rate increases, the Nyquist frequency (half of the interference pattern frequency) also increases. This holds true for the DUT interference pattern frequency which increases accordingly. Therefore, regardless of the repetition rate, the impulse within the FFT response will always appear at the same digital frequency (specific data point).

$$
\begin{gathered}
f_{N}=\frac{f_{r}}{2} \frac{B W}{\Delta \lambda_{i n t}} \\
\Delta \lambda_{\text {int }}=\Delta f_{\text {int }} \frac{\lambda_{c}^{2}}{c}
\end{gathered}
$$


The FFT data points provide the necessary frame of reference because of the relationship shown in equation (6.3) which provides the necessary conversion between the interferometer frequency difference and the emulated distance.

$$
d=\frac{c}{2 \Delta f_{\text {int }}}
$$

According to this relationship, the measured distance is approximately $3.8 \mathrm{~mm}$, which occurs when $F_{d}=\frac{113}{512}=0.221$. To create a conversion relationship, when $F_{d}=0$, the measured distance is assumed to be equal to the zero path length difference $(0 \mathrm{~mm})$. This conversion enables the following capability assessment in table (6.1), particular the resolution performance.

Table 6.1: Capability assessment of spectral integrity of $39 \mathrm{GHz}$ DUT

\begin{tabular}{c|c|c|c|c} 
39 GHz DUT & $\begin{array}{c}\text { No Stitching Errors } \\
\text { Removed at 1 kHz } \\
\text { (Figure 6.5) }\end{array}$ & $\begin{array}{c}\text { All Stitching Errors } \\
\text { Removed at 1 kHz } \\
\text { (Figure 6.6) }\end{array}$ & $\begin{array}{c}\mathbf{2 0 0 ~ H z} \\
\text { (Figure 6.7) }\end{array}$ & $\begin{array}{c}\mathbf{3 ~ k H z} \\
\text { (Figure 6.8) }\end{array}$ \\
\hline \hline $\begin{array}{c}\text { Resolution } \\
\text { SNR }\end{array}$ & $\approx 168 \mu \mathrm{m}$ & $\approx 47 \mu \mathrm{m}$ & $\approx 45 \mu \mathrm{m}$ & $\approx 54 \mu \mathrm{m}$ \\
$16.54 \mathrm{~dB}$ & $29.89 \mathrm{~dB}$ & $31.66 \mathrm{~dB}$ & $18.75 \mathrm{~dB}$
\end{tabular}

These results validate the claim that with a decreased repetition rate when stitching errors are removed, the resolution and signal to noise ratio improves. The obvious tradeoff is a slower measurement response and increased processing effort to remove stitching errors. The maximum attainable resolution with this system is in accordance to the theoretical expectation according to equation (6.4). For medical imaging applications, the attained resolution of $45 \mu \mathrm{m}$ is approximately four times the size of the diameter of a red blood cell.

$$
\delta R=\frac{c}{2 B}=\frac{3 \times 10^{8}}{2\left(4.4803 \times 10^{12}\right)}=33.5 \mu \mathrm{m}
$$




\section{CHAPTER 7 - Conclusion and Future Work}

This thesis presented a method for automatically calibrating SG-DBR lasers for optical coherence tomography applications. The automated calibration program was successful in creating four synchronized waveforms which produce a linear frequency ramp. Compared to previous manual efforts, laser bandwidth, power leveling and most importantly, calibration time improved. Manual calibrations would normally last up to 48 hours, which was dramatically reduced to approximately 10 hours. Experimentally testing the sweeping laser in a SS-OCT system resulted in the expected high resolution capabilities ideal for the researched medical imaging applications.

To begin this process in chapter 4 , two-dimensional and three-dimensional gain medium voltage characteristics were analyzed. This analysis showed that instantaneous voltage changes occur during wavelength mode changes. A method for sensing the appropriate threshold level was conducted which provides the necessary foundation to create an automated laser calibration.

In chapter 5, the automated laser calibration algorithm was thoroughly explained. The goal of this implementation was to create a fully automated calibration adaptable for any SG-DBR laser that would reduce calibration time. This program was successful in achieving this objective which located centers of tuning pads with the gain medium sensing information provided in chapter 4 . The linearity of the frequency ramp was assessed by determining the distribution of data for the time between zero-crossings in the output interference pattern. A qualitative determination of stitching imperfections was also conducted which led to a method to reduce these effects in chapter 6. 
Experimental OCT tests were conducted to reveal the overall capabilities of this tunable laser source. The effects of stitching imperfections were reduced by implementing a method to create a self-generating sampling clock. This clock generation software allows for a manual removal of stitching errors which improved the spectral response. An analysis of the repetition rate as a function of spectral integrity was also performed. The experimental capabilities of this prototype system resulted in a high distance resolution less than $45 \mu \mathrm{m}$ and a signal to noise ratio of over $30 \mathrm{~dB}$. This system provides an improved solution to the researched medical imaging applications.

Additional research includes an extensive and more thorough analysis of the experimental OCT system. Creating software to model the entire system would be beneficial to conducting this analysis. Also, cascading the $\mathrm{C}$ and L-Band lasers together to increase the bandwidth would significantly improve the resolution. Future research also includes optimizing the variable increment size for the gain medium voltage sensing, which could reduce the automated calibration time. A tradeoff exists between correctly sensing mode changes and calibration time, which could pose a challenge. A faster digital voltmeter could also help in this effort.

In summation, this thesis demonstrated that the gain medium voltage characteristics provide a method for automatically sensing wavelength differences which allowed for a successful automated calibration implementation. It also demonstrated that the JDS Uniphase SG-DBR laser provides a solution suitable for high-resolution medical imaging systems. 


\section{Bibliography}

[1] "Optical Coherence Tomography Protocol," Fundus Photograph Reading Center. Department of Ophthalmology and Visual Sciences University of Wisconsin Madison, 2001, 25 March 2008, <http://eyephoto.ophth.wisc.edu/ResearchAreas/oct/oct1.htm>

[2] M. Musa and S. Salous, "Ambiguity elimination in HF FMCW radar systems," Department of Electrical Engineering \& Electronics, University of Manchester Institute of Science \& Technology, 12 Jan. 2000.

[3] Shane O'Connor, "High Speed Wavelength Tuning of SG-DBR Lasers for Light Detection and Ranging and Optical Coherence Tomography," Electrical Engineering Department, California Polytechnic State University, San Luis Obispo. June 2008.

[4] David L. Price, Janet P. De Wilde, PhD, Annie M. Papadaki, Jane S. Curran, Richard I. Kitney, PhD, "Investigation of acoustic noise on 15 MRI scanners from $0.2 \mathrm{~T}$ to 3 T" Journal of Magnetic Resonance Imaging, vol. 13, no. 2, pp. 288293, Jan. 2001.

[5] Mark Brezinkski, MD, PhD, Optical Coherence Tomography, Principles and Applications, San Diego, CA: Academic Press, 2006.

[6] S.L. Myers, K. Dines, D.A. Brandt, K.D. Brandt, and M.E. Albrecht (1995). "Experimental assessment by high frequency ultrasound of articular cartilage thickness and osteoarthritic changes" J. Rheumatol. 22, 109-116.

[7] Vinay Kumar, Abul K. Abbas, Nelson Fausto, Richard N. Mitchell, Robbins Basic Pathology (8th ed.), Saunders Elsevier. 2007.

[8] J.H. Culbertson, R.P. Rand, and M.J. Jurkiewicz (1990). "Advances in microsurgery.” Adv. Surg. 23, 57-88.

[9] William T. Silfvast, Laser Fundamentals, New York: Cambridge University Press, 2004.

[10] "Stimulated Emission" Encyclopedia of Laser Physics and Technology. RPPhotonics, 2009. Web. 02 May 2010.

<http://www.rp-photonics.com/stimulated_emission.html>

[11] Manijeh Razeghi, Fundamentals of Solid State Engineering, New York, New York: Springer Science+Business Media LLC, 2007.

[12] Ronald F. Brown, Solid State Physics, An Introduction for Scientists and Engineers, San Luis Obispo, CA: El Corral Publications, 2006. 
[13] “LED Primer” JPSA Laser, 2010. Web 02 May 2010. <http://www.jpsalaser.com/apps_led.html>

[14] Dennis Derickson, Mike Bernacil, Andrew DeKelaita, Ben Maher, Shane O’Connor, Matthew N. Sysak, and Leif Johanssen, "SGDBR single-chip wavelength tunable lasers for swept source OCT," Electrical Engineering Department, California Polytechnic State University, San Luis Obispo.

[15] Brandon J George, "Swept-Frequency Sampled Grating Distributed Bragg Reflector Lasers Optimized for Optical Coherence Tomography Applications," Electrical Engineering Department, California Polytechnic State University, San Luis Obispo. December 2009.

[16] C.E. Mungan, "Faraday Isolators and Kirchhoff's Law: A Puzzle" Web 01 June 2010.

〈http://www.usna.edu/Users/physics/mungan/Scholarship/FaradayIsolators.pdf〉

[17] Gert Scarlet, Geert Morthier, and Roel Baets, "Control of Widely Tunable SGDBR Lasers for Dense Wavelength Division Multiplexing," Journal of Lightwave Technology, vol. 18, pp. 1128-1138, 8 Aug. 2000.

[18] "Linewidth" Encyclopedia of Laser Physics and Technology. RP- Photonics, 2009. Web 01 Apr. 2010.

<http://www.rp-photonics.com/linewidth.html>

[19] Dennis Derickson, Fiber Optic Test and Measurement, Upper Saddle River, New Jersey: Prentice Hall, 1998.

[20] S. L. Woodward, I. M. I. Habbab, T. L. Koch, and U. Koren, "The side-modesuppression ratio of a tunable DBR laser," IEEE Photon. Technol. Lett., vol. 2, no. 12, pp. 854-856, Dec. 1990. 


\section{Appendix A - JDSU SG-DBR Pin-out and Operating Current Levels}

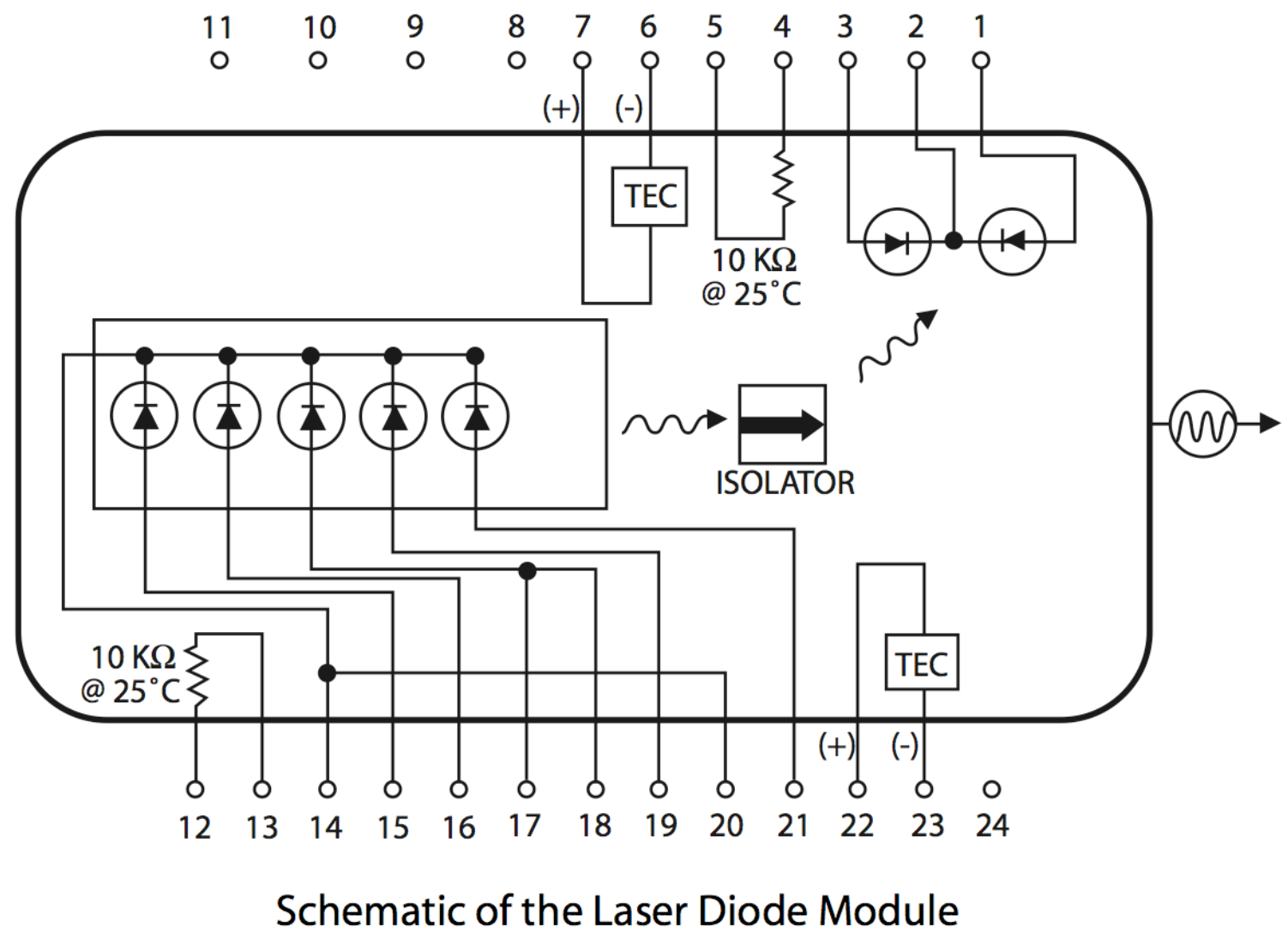




\begin{tabular}{|c|c|c|}
\hline Pin Number & Description & Maximum Safe Operating Current \\
\hline 1 & Etalon Photodiode Anode (-) & \\
\hline 2 & Photodiode Common Cathode (+) & \\
\hline 3 & Reference Photodiode Anode (-) & \\
\hline 4 & Locker Thermistor & \multirow{2}{*}{$100 \mu \mathrm{A}$} \\
\hline 5 & Locker Thermistor & \\
\hline 6 & Locker TEC (-) & \\
\hline 7 & Locker TEC (+) & \\
\hline 8 & $\mathbf{N} / \mathbf{C}$ & \\
\hline 9 & $\mathrm{~N} / \mathrm{C}$ & \\
\hline 10 & $\mathrm{~N} / \mathrm{C}$ & \\
\hline 11 & $\mathrm{~N} / \mathrm{C}$ & \\
\hline 12 & Laser Thermistor & \multirow{2}{*}{$100 \mu \mathrm{A}$} \\
\hline 13 & Laser Thermistor & \\
\hline 14 & Laser Cathode (-) & \\
\hline 15 & Back Mirror Anode (+) & $60 \mathrm{~mA}$ \\
\hline 16 & Phase Anode (+) & $20 \mathrm{~mA}$ \\
\hline 17 & Gain Anode (+) & \multirow{2}{*}{$150 \mathrm{~mA}$} \\
\hline 18 & Gain Anode (+) & \\
\hline 19 & Front Mirror Anode (+) & $35 \mathrm{~mA}$ \\
\hline 20 & Laser Cathode (-) & \\
\hline 21 & SOA Anode (+) & $195 \mathrm{~mA}$ \\
\hline 22 & Laser TEC (+) & \\
\hline 23 & Laser TEC (-) & \\
\hline 24 & $\mathrm{~N} / \mathrm{C}$ & \\
\hline
\end{tabular}

